\title{
Astrophysical and Astrochemical Insights into the Origin of Life
}

P. Ehrenfreund, L. Becker, J. Blank, J. Brucato, L. Colangeli, S. Derenne, D. Despois, A. Dutrey, H. Fraaije, W. Irvine, A. Lazcano, T. Owen, R. Robert

\section{April 30, 2002}

U.S. Department of Energy

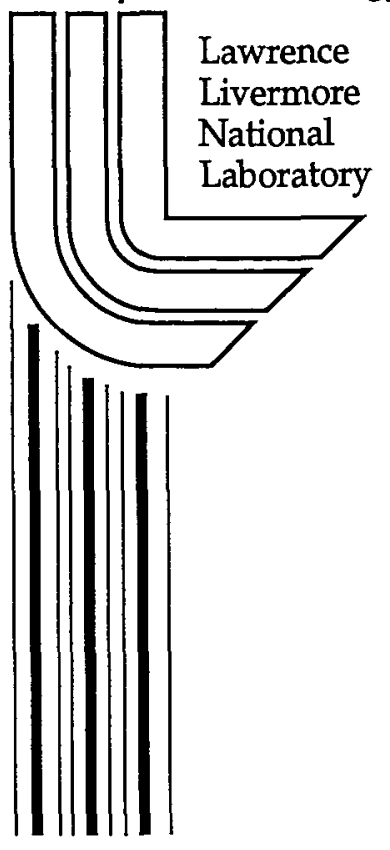




\section{DISCLAIMER}

This document was prepared as an account of work sponsored by an agency of the United States Government. Neither the United States Government nor the University of California nor any of their employees, makes any warranty, express or implied, or assumes any legal liability or responsibility for the accuracy, completeness, or usefulness of any information, apparatus, product, or process disclosed, or represents that its use would not infringe privately owned rights. Reference herein to any specific commercial product, process, or service by trade name, trademark, manufacturer, or otherwise, does not necessarily constitute or imply its endorsement, recommendation, or favoring by the United States Government or the University of California. The views and opinions of authors expressed herein do not necessarily state or reflect those of the United States Government or the University of California, and shall not be used for advertising or product endorsement purposes.

This work was performed under the auspices of the U. S. Department of Energy by the University of California, Lawrence Livermore National Laboratory under Contract No. W-7405-Eng-48.

This report has been reproduced directly from the best available copy.

Available electronically at http://www.doc.gov/bridge

Available for a processing fee to U.S. Department of Energy

And its contractors in paper from

U.S. Department of Energy

Office of Scientific and Technical Information

P.O. Box 62

Oak Ridge, TN 37831-0062

Telephone: (865) 576-8401

Facsimile: (865) 576-5728

E-mail: reports@adonis.osti.gov

Available for the sale to the public from

U.S. Department of Commerce

National Technical Information Service

5285 Port Royal Road

Springfield, VA 22161

Telephone: (800) 553-6847

Facsimile: (703) 605-6900

E-mail: orders@ntis.fedworld.gov

Online ordering: http://www.ntis.gov/ordering.htm

\section{OR}

Lawrence Livermore National Laboratory

Technical Information Department's Digital Library

http://www.llnl.gov/tid/Library.html 


\title{
Astrophysical and Astrochemical Insights into the Origin of Life
}

\author{
invited contribution to \\ Reports on Progress in Physics
}

\section{ISSI (International Space Science Institute)Team: \\ "Prebiotic Matter in Space"}

in alphabetical order

P. Ehrenfreund ${ }^{(1,2)}$, L. Becker ${ }^{(3)}$, J. Blank $^{(4)}$, J. Brucato ${ }^{(5)}$, L. Colangeli ${ }^{(5)}$, S. Derenne ${ }^{(6)}$, D. Despois ${ }^{(7)}$, A. Dutrey ${ }^{(8)}$, H. Fraaije ${ }^{(2)}$, W. Irvine ${ }^{(9)}$, A. Lazcano ${ }^{(10)}$, T. Owen ${ }^{(11)}$, F. Robert ${ }^{(12)}$

${ }^{(1)}$ Leiden Observatory, P O Box 9513, 2300 RA Leiden, The Netherlands

(2) Soft Matter / Astrobiology Laboratory, Leiden Institute of Chemistry, PO BOX 9502, 2300 RA Leiden

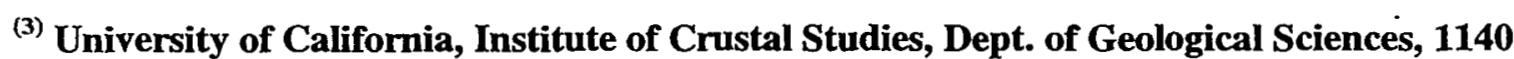
Girvetz Hall, Santa Barbara, CA 93106, USA

${ }^{(4)}$ Lawrence Livermore National Laboratory, H-Division/Shock Physics Group, P.O. Box 808, L-415 Livermore, CA 94551, USA

${ }^{(5)}$ INAF-Osservatorio Astronomico di Capodimonte via Moiariello 16, I-80131, Napoli, Italy.

(6) Laboratoire de Chimie Bioorganique et Organique Physique, UMR CNRS 7573, Ecole Nationale Superieure de Chimie de Paris, 11, rue Pierre et Marie Curie 75231 Paris Cedex 05, FRANCE

(7) Observatoire de Bordeaux (INSU/CNRS), B.P. 89, F-33270 Floirac, France

${ }^{(8)}$ LAOG, BP 53, F-38041 Grenoble Cedex 9, France

(9) Astronomy Department, 619 Lederle Graduate Research Center, University of Massachusetts, Amherst, MA 01003, USA

${ }^{(10)}$ Facultad de Ciencias, UNAM, Apdo. Postal 70-407, Cd. Universitaria, 04510 Mexico

D.F., Mexico

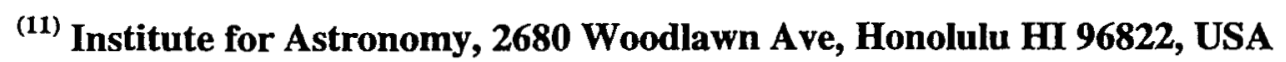

${ }^{(12)}$ Laboratoire de Mineralogie, Museum National d'Histoire Naturelle, 61 rue Buffon 75005 Paris, FRANCE 


\begin{abstract}
Nucleosynthesis of heavy elements such as carbon in stars allowed the formation of organic molecules in space, which appear to be widespread in our Galaxy. The physical and chemical conditions - including density, temperature, ultraviolet radiation and energetic particles - determine reaction pathways and the complexity of organic molecules in different space environments. Dense interstellar clouds are the birth sites of stars of all masses and their planetary systems. During the protostellar collapse, gaseous and solid interstellar organic molecules are integrated into protostellar disks from which planets and smaller solar systen bodies form. After the formation of the planets in our own solar system, 4.6 billion years ago, all of them, including the Earth, were subjected to frequent impacts for several hundred million years. First indications for life on Earth are dated 3.8-3.5 billion years ago. That life arose very shortly after this heavy bombardment phase raises many questions. A prebiotic non-reducing atmosphere predicts that building blocks of macromoleculessuch as amino acids, sugars, purines and pyrimidines were not formed in abundance. A part of the prebiotic organic content of the early Earth could have been delivered by asteroids, comets and smaller fragments such as meteorites and interplanetary dust particles (IDPs).
\end{abstract}

Current experimental research investigating the origin of life is focussed on the spontaneous formation of stable polymers out of monomers. However, understanding the spontaneous formation of structure is not enough to understand the formation of life. The introduction and evolution of information and complexity is essential to our definition of life. The formation of complexity and the means to distribute and store information is currently investigated in a number of theoretical frameworks; such as evolving algorithms, chaos theory, and modern evolution theory.

In this article we review the physical and chemical processes that form and shape organic matter in space. In particular we discuss the chemical pathways of organic matter in the interstellar medium, their evolution in protoplanetary disks and their integration into solar system material. Furthermore we investigate the role of impacts and the delivery of organic matter to the prebiotic Earth. Processes that may have assembled prebiotic molecules to produce the first genetic material and ideas about the formation of complexity in chemical networks are discussed. 


\section{Introduction}

The story of astrobiology in the universe begins with the synthesis of the elements that play key roles in life as we know it: hydrogen, carbon, oxygen, nitrogen, sulfur and phosphorus. Other elements are required by specific terrestrial life forms, or perhaps by the Earth's biosphere as an interacting whole, but might be replaceable by different chemical elements under somewhat different initial conditions.

Hydrogen is effectively primordial, having been formed from the soup of quarks that filled the universe in the earliest stage of the Big Bang. All the other chemical elements that are featured in terrestrial biochemistry were manufactured by nucleosynthesis during the course of stellar evolution. Carbon, the basis of organic chemistry and the lightest of the "biogenic" elements, is produced by the so-called triple- $\alpha$ process $\left(3 \times{ }^{4} \mathrm{He} \rightarrow{ }^{12} \mathrm{C}\right.$ ) in the cores of stars more massive than half a solar mass, after these stars have fused a significant fraction of their core hydrogen into helium and evolved off the Main Sequence (see review by Trimble, 1997, and Figure 1). In the same cores, some carbon is further processed to the principal isotope of oxygen by ${ }^{12} \mathrm{C}+{ }^{4} \mathrm{He} \rightarrow{ }^{16} \mathrm{O}$. The fraction of the $\mathrm{C}$ and $\mathrm{O}$ produced that is delivered to the interstellar medium, from which it can be incorporated into new stars (such as the sun once was), depends on the mass of the synthesizing star and its resulting final fate (e.g., Matteucci, 1991).

In the cores of evolving, massive ( $\mathrm{M}>10$ solar masses) stars, heavy element nuclear "burning" produces the most abundant isotopes of sulfur $\left({ }^{32} \mathrm{~S}\right)$ and phosphorus $\left({ }^{31} \mathrm{P}\right)$. It is easy to see that ${ }^{32} \mathrm{~S}$ is built from $8 \times{ }^{4} \mathrm{He}$ units, although the detailed process is more complicated than simple successive addition of $\alpha$ particles to carbon or oxygen, because there are bottle necks along the route (Trimble, 1997). A primary source of phosphorus in these stars is the reaction ${ }^{16} \mathrm{O}+{ }^{16} \mathrm{O} \rightarrow{ }^{31} \mathrm{P}+{ }^{1} \mathrm{H}$ (e.g., Hansen \& Kawaler, 1994).

In contrast to the "primary" production of $\mathrm{C}$ and $\mathrm{O}$, nitrogen is a "secondary". product, in the sense that its synthesis requires the presence of pre-existing $\mathrm{C}$ or $\mathrm{O}$ from earlier generations of stars. In particular, nitrogen is a by-product of hydrogen fusion to helium through the $\mathrm{CN}$ or the CNO cycles in the cores of massive stars and the shells of evolved lower mass stars, where $\mathrm{C}$ or $\mathrm{O}$ act as catalyst for the hydrogen "burning" (e.g., Shu, 1981).

The step from atomic nuclei to molecules begins with the expulsion of nucleosynthetic products into the interstellar medium (ISM) by stellar winds, planetary nebula ejection, and supernova explosions. These events include the local formation of gas phase molecules and dust grains, the latter being predominantly organic or silicatic when the progenitor star is either carbon- or oxygen-rich. The molecules, which can include a rich array of organic species (e.g., Guelin et al., 2000; Cernicharo, 2000), are probably mostly dissociated before reaching the shelter of a dense interstellar cloud. In contrast, both silicate and organic dust grains can survive for extended periods in the diffuse ISM and may then be incorporated into dense clouds, where they may acquire icy mantles, be processed in various ways, and serve as sites for molecular synthesis. 
Figure 1 (put here)

Interstellar clouds are the birthsites of stars of all masses. The gravitational collapse of interstellar dust and gas triggered by cloud instabilities leads to the formation of stars, which may be accompanied by planetary systems. Interstellar species thus provides the raw material from which planets and small solar system bodies form. To date many open questions remain in order to reconstruct the formation of our solar system in detail; it resembles a puzzle where many pieces are still missing and some pieces may have been put in the wrong places, as a result. The physical and chemical processes that have modified the original material and shaped the structure of our planetary system are partly unknown. However, knowledge of the composition of interstellar clouds and solar system bodies has strongly increased in recent years due to sensitive instruments mounted on ground-or space-based facilities. Analysis of these cosmic materials - be they gaseous or solid - remains a vital tool for monitoring their evolution in space and tracing their path from star-forming regions to planetesimals and solar system bodies. Interplanetary dust and remnant planetesimals not incorporated into planets delivered material to the early planets and still do in the form of swept up dust and meteorites, which represent fragments of small solar system bodies. Part of this stuff included organic matter, which may have been the crucial raw material triggering an increase in the complexity of chemical reactions that ultimately led to the origin of life.

On the other hand it may very well be that life emerged on the early Earth simply due to a combination of the right local conditions and without any "help" from space. The step from the presence of organic species, such as $\mathrm{H}_{2} \mathrm{CO}$, amino acids or polycyclic aromatic hydrocarbons (PAHs) to a self-replicating protected cell structure is immense and far beyond the field of astrophysics. This review provides insights into the evolution and distribution of organic material in space, on exogenous delivery processes and their possible relevance to the basic theories for life's emergence. For this purpose we have compiled the inventory of organics in interstellar clouds, comets and meteorites, as well as the limited data available for protostellar disks. We discuss laboratory experiments for the identification of compounds in different environments and methods used to establish a link between them, such as isotopic data from the interstellar medium and throughout the solar system. We investigate impact properties and delivery processes and the necessary conditions for the origin of life. We conclude with a discussion of future perspectives in astronomy and biochemistry that might help to reveal some of the steps that led to life on Earth and possibly other planets as well. 


\section{The chemistry of interstellar clouds}

The ISM in our Milky Way and in other galaxies includes regions of differing density, temperature, and radiation intensity. For perhaps two decades, debate has continued as to the volume fraction of the ISM occupied by hot $\left(\sim 10^{6} \mathrm{~K}\right)$, very low density cavities created by supernova remnants; "warm" $\left(\sim 10^{4} \mathrm{~K}\right)$ "intercloud" gas that is either mostly ionized or mostly neutral; and interstellar "clouds", identified by specific velocity components observed in absorption or emission (e.g., Shull, 1987). The clouds, which are thought to occupy only a minor volume fraction of the overall ISM, are in turn divisible into the so-called diffuse clouds which are characterized by visual extinctions $A v \sim 1 \mathrm{mag}$, densities of the order of 100 atoms $\mathrm{cm}^{-3}$, and temperatures $\sim 100 \mathrm{~K}$; dense clouds with $\mathrm{Av}>5 \mathrm{mag}$, densities $>10^{3} \mathrm{~cm}^{-3}$, and temperatures as low as $10 \mathrm{~K}$; and the translucent clouds that are intermediate in extinction, density and temperature (e.g., van Dishoeck 1998).

For our purposes the dense clouds are the most interesting, since it is here that a wide variety of organic molecules have been identified. These regions, also referred to as "molecular clouds", may again be subdivided into cold, quiescent clouds; clouds where low mass stars form; and cores where high-mass star formation is underway (the so-called "hot cores"). In these dense clouds molecular synthesis takes place both in the gas phase and on (or in) the grains (cf. reviews by Langer et al., 2000; Ehrenfreund \& Charnley, 2000; Herbst, 2000; Millar, 1997; Hartquist \& W Williams, 1995). The basic, and by far the most abundant constituent, molecular hydrogen $\left(\mathrm{H}_{2}\right)$, is formed on grain surfaces. Those gas phase species that have been identified are listed in Table 1, which also includes molecules detected in the envelopes expelled by evolved stars. The vast majority of these species were detected at short radio wavelengths, where they have pure rotational transitions. The heterodyne techniques employed allow very high frequency resolution, minimizing the possibility of accidental line coincidences, although the richness of the spectra can still lead to confusion and misidentifications (cf. the careful approach of Dickens et al., 1997, with the problems identified by Ellder et al., 1980). The non-polar molecules were observed via ro-vibrational transitions in the infrared, typically in absorption against bright background sources such as luminous young stars embedded in the clouds.

Chemical processes in the gas phase in cold, quiescent clouds are predominantly ionmolecule reactions, powered primarily by cosmic ray ionization of $\mathrm{H}_{2}$. Calculated abundances of observed molecules mostly match the observations rather well (e.g., Pratap et al., 1997; Dickens et al., 2000), although the agreement depends on factors such as the age of the cloud and the relative abundance of $C$ and $O$ in the gas. Note that physical parameters such as temperature and density may be determined directly from the molecular line emission. By far the most abundant molecule in these clouds is $\mathrm{H}_{2}$, and the most abundant carbon-containing species is $\mathrm{CO}$, with $\mathrm{CO} / \mathrm{H}_{2}$ of order $10^{-4}$. Less abundant by another factor of $10^{-3}$ to $10^{-4}$ are molecules such as $\mathrm{H}_{2} \mathrm{CO}$, $\mathrm{HCN}, \mathrm{NH}_{3}$, and $\mathrm{CH}_{3} \mathrm{CCH}$. The chemistry of the cold "molecular clouds" is characterized by the presence of species normally considered to be highly reactive, including radicals, ions, and higher energy isomers, as well as by very unsaturated species such as cyanopolyynes and related linear carbon chains (see Table 1).

Table 1 (put here) 
The presence of these de-hydrogenated species in an overall very reducing environment is a result of the kinetics of low temperature, low density reactions in the gas phase; in particular, reactions that add carbon to molecules tend to result in the loss of hydrogen, and many hydrocarbon ions have barriers to re-hydrogenation by reaction with $\mathrm{H}_{2}$ (e.g., Herbst et al., 1983). The large values for the $\mathrm{HNC} / \mathrm{HCN}$ abundance ratio have also been considered to be a classic indicator of ion-molecule chemistry, making the detection of HNC in comets of special interest (e.g., Irvine et al., 1998a).

As cold clouds evolve, and particularly as cores collapse as part of star formation, molecules begin to freeze out onto the grains, forming icy mantles over the underlying silicates and organics (Ehrenfreund \& Schutte 2000). An active chemistry takes place on grain surfaces and can lead to the formation of complex interstellar molecules. At $10 \mathrm{~K}$ only $\mathrm{H}, \mathrm{D}, \mathrm{C}, \mathrm{O}$ and $\mathrm{N}$ atoms have sufficient mobility to interact on the surfaces of grains. The formation of the simplest mantle molecules $\left(\mathrm{H}_{2} \mathrm{O}\right.$, $\mathrm{NH}_{3}, \mathrm{CH}_{4}$, etc.) can be explained by simple exothermic hydrogen addition reactions. However, the presence of more complex molecules (e.g. $\mathrm{CH}_{3} \mathrm{OH}$ ) and various organics requires reaction processes that are acting on and in the icy mantles (Brown \& Charnley, 1990). Water ice is the most abundant ice component formed in icy grain mantles and abundances of other species are therefore scaled relative to water ice. Highly volatile species such as $\mathrm{CO}, \mathrm{O}_{2}$ and $\mathrm{N}_{2}$ sublimate around or below $20 \mathrm{~K}$. This implies that successive layers of ice are formed with different ice compositions according to the prevailing temperatures and gas pressures (or absolute densities) in protostellar regions. Hydrogen-rich ices (polar ices), dominated by $\mathrm{H}_{2} \mathrm{O}$ ice, are formed when $\mathrm{H}$ is abundant in the interstellar gas. They contain besides water ice: $\mathrm{CO}, \mathrm{CO}_{2}, \mathrm{CH}_{4}, \mathrm{NH}_{3}, \mathrm{CH}_{3} \mathrm{OH}$, and possibly traces of $\mathrm{HCOOH}$ and $\mathrm{H}_{2} \mathrm{CO}$. Such polar ices generally evaporate around $100 \mathrm{~K}$ under astrophysical conditions and can therefore survive in higher temperature regions close to the star (Tielens \& Whittet 1997). Apolar or hydrogen-poor ices are formed far away from the proto-star and are composed of molecules with high volatility (evaporation temperatures of $<20 \mathrm{~K}$ ) such as $\mathrm{CO}, \mathrm{O}_{2}$ and $\mathrm{N}_{2}$ (Ehrenfreund et al. 1997). Trace species such as $\mathrm{OCS}, \mathrm{H}_{2} \mathrm{CO}$, $\mathrm{HCOOH}, \mathrm{CH}_{4}$, and $\mathrm{OCN}^{-}$have been observed with the Infrared Space Observatory (ISO) near some protostars and are characterized by abundances between less than a percent to a few percent relative to water ice (Ehrenfreund \& Charnley 2000, Gibb et al. 2000a, Keane et al. 2001).

Currently the largest molecule observed on interstellar grain mantles is $\mathrm{CH}_{3} \mathrm{OH}$. Methanol, together with $\mathrm{NH}_{3}$, is a key molecule in reaction pathways leading to further complexity. The abundance of both molecules is strongly varying and/or debated (Dartois et al. 1999, Lacy et al. 1998, Dartois \& d'Hendecourt 2001, Gibb et al. 2001). OCS is currently the only sulphur-containing species identified in interstellar ices, and has an abundance of $0.2 \%$ relative to water ice (Palumbo et al. 1995, 1997, d'Hendecourt et al. 1996). Among the most important species, whose presence and abundances are difficult to determine, are the infrared inactive molecules $\mathrm{H}_{2}, \mathrm{O}_{2}$ and $\mathrm{N}_{2}$. It has been shown that the infrared transitions of those molecules become weakly infrared active in the solid (Ehrenfreund et al. 1992a, Sandford et al. 1993). None of those molecules has up to now been detected in interstellar ices. 
Upper limits for $\mathrm{O}_{2}$ and $\mathrm{N}_{2}$ (transitions at 6.45 and $4.28 \mu \mathrm{m}$, respectively) have been recently reported by Vandenbussche et al. (1999) and Sandford et al. (2001). With the help of recent ISO observations and ground-based data it is clear that the observations sample the spectral signature of many different grain populations in the line of sight through a molecular cloud. Additionally, those grain populations are mixed within regions of different temperature and density conditions. Grain chemistry may become even more complex in high-energy environments. In brief, what we observe is a dynamical system within which the chemical composition is altered from one region to another.

The energy released in processes related to star formation can cause icy grain mantles to sublimate, releasing back to the gas both the unprocessed species originally formed in the cold gas, and those molecules formed on the grains. The formation of massive stars produces the largest energy input to the surrounding cloud, and the resulting "hot cores" are the richest sources of complex (by astronomical standards) organic molecules in the ISM. It is here that reactions of sublimated species with pre-existing gas phase molecules are thought to give rise to molecules such as $\left(\mathrm{CH}_{3}\right)_{2} \mathrm{O}$ and $\mathrm{HCOOCH}_{3}$, and potentially to heavier "biogenic" species such as (yet unconfirmed) glycine (Charnley et al. 2001).

The heavier observed species seem to often be confined to regions of small angular size in the hot cores, and abundances are consequently difficult to determine with the limited angular resolution currently available to radio astronomers (Snyder 1997). However, estimates may be made by applying "rotation diagrams" to observations of multiple transitions that cover a range of energies above the ground state (e.g., Nummelin et al. 2000). The results for interesting organic molecules can be much higher than the abundances found in cold clouds, e.g., (by number relative to $\mathrm{H}_{2}$ ) as high as $10^{-5}$ for $\mathrm{CH}_{3} \mathrm{OH}$ and $>10^{-8}-10^{-7}$ for $\mathrm{CHC}_{3} \mathrm{H}_{2} \mathrm{CN}$ and $\mathrm{HCOOCH}_{3}$ (Gibb et al. 2000b), see Figure 2.

\section{Figure 2 (put here)}

A key characteristic of the chemistry in quiescent clouds, which is largely preserved in observed hot cores, is very large isotopic fractionation, particularly for $\mathrm{D} / \mathrm{H}$. The kinetics in cold clouds that concentrates deuterium in molecules other than $\mathrm{HD}$ is well understood (e.g., van Dishoeck, 1998). Enhancements relative to the cosmic D/H abundance ratio $\left(-2 \times 10^{-5}\right)$ by factors up to $10^{4}$ for some species (such as $\mathrm{C}_{3} \mathrm{H}_{2}$ ) are observed (Irvine \& Knacke 1989). Even doubly deuterated species, specifically $\mathrm{D}_{2} \mathrm{CO}$ and $\mathrm{NHD}_{2}$, have been detected (e.g., Turner 1990; Roueff et al., 2000, Ceccarelli et al. 2002). The preservation of high fractionation in the warmer hot core gas is more difficult to understand, but presumably reflects the state of the sublimated gas and the relatively short lifetime of the hot core evolutionary phase (Rodgers \& Millar 1996; Roberts \& Millar 2000). It is interesting that the chemistry of comets, at least as manifested by the composition of the coma, bears a number of close similarities to that of dense interstellar clouds (e.g. Irvine et al. 2000; Irvine \& Bergin 2000 ). This is the case for the relative abundance of some volatiles, the large isotopic fractionation for $\mathrm{D} / \mathrm{H}$, the presence of high energy species such as $\mathrm{HNC}$, and possibly the non-equilibrium values of ortho/para ratios (see section 6). 


\section{Cosmic Dust}

Cosmic matter condenses primarily in the extended atmospheres of evolved stars (e.g., red giants), that slowly lose mass, or in dramatic expulsion events from novae and supernovae. The elemental abundance available in the parent stars determines the chemical composition of formed solids: carbon species primarily condense around carbon-rich stars, while silicates dominate in oxygen-rich stellar atmospheres. Here, oxygen, in excess to that locked in $\mathrm{CO}$ bearing molecules, forms Si-O networks involving cations, such as $\mathrm{Mg}$ and Fe. Once injected into the interstellar medium, materials enter a cycle, between diffuse and dense clouds, where various energetic processes contribute to modify the original nature of compounds and new species may condense, despite the low density conditions. The complex evolution of chemistry, structure and physical properties (e.g., morphology) of materials is driven by interactions with gas and by processes such as heating, UV radiation, ion bombardment and shocks.

The diffuse interstellar medium is less dense $\left(10^{3} \mathrm{~g} \mathrm{~cm}^{-3}\right)$ and characterized by higher temperatures $(\sim 100 \mathrm{~K})$ than the outer regions of stellar envelopes. UV photons can penetrate easily and photochemistry drives the molecular chemistry in these regions. Spectroscopy from the UV to radio wavelength reveals a diversity of gasesous and solid species. Only diatomic molecules such as $\mathrm{CO}, \mathrm{CH}, \mathrm{CN}$, and $\mathrm{H}_{2}$ have been securely identified in the diffuse clouds, although there is clearly complex organic matter present in particulate form ("dust" or "grains"), which on the low-mass side probably merges into the large organic molecules referred to generically as polycyclic aromatic hydrocarbons (PAHs). The latter include both neutral and ionized species, of varying degrees of hydrogenation, depending on the radiation environment, and possibly heterogeneous atoms such as $\mathrm{N}$ and $\mathrm{O}$. Simple polyatomic species such as $\mathrm{HCN}, \mathrm{HCO}^{+}, \mathrm{CO}$ and $\mathrm{C}_{3} \mathrm{H}_{2}$ appear in the translucent clouds, which serve as excellent laboratories for testing models of gas phase chemistry in the ISM.

The cosmic carbon abundance is an important criterion when considering the inventory of organics in the Universe. Measurements of solar carbon values give 355 \pm 50 carbon atoms per $10^{6} \mathrm{H}$ atoms (ppm). Recent measurements using the Goddard High Resolution Spectrograph (GHRS) on the Hubble Space Telescope indicate interstellar carbon abundances that are only two-thirds of the solar values (Cardelli et al. 1996). Snow \& Witt (1995) constrain the cosmic carbon abundance to $225 \pm 50$ ppm with a mean gas phase abundance of $\sim 140 \pm 20 \mathrm{ppm}$. Approximately $20 \%$ of the cosmic carbon is incorporated in gaseous $\mathrm{CO}$ (the most abundant molecule in space after $\mathrm{H}_{2}$ ) and a few \% in ices prevailing in dense clouds (see section 1). It is unclear where the remaining cosmic carbon is hidden. Astronomical observations suggest that a dominant fraction might be present in large carbonaceous species, namely up to 15 $\%$ in PAHs and up to $50 \%$ in the form of macromolecular carbon in grains, such as amorphous carbon and complex kerogen-type material. Other organic compounds observed or suspected in diffuse clouds are carbon-chains, diamonds and fullerenes (Ehrenfreund \& Charnley, 2000). 


\subsection{Laboratory studies tracing cosmic materials in diffuse clouds}

Ground-based and space-born (e.g., Hubble Space Telescope, HST, and Infrared Space Observatory, ISO) observations over the widest spectral range represent the primary source of information about the properties and evolution of materials in space. Identification of typical spectral signatures in the most relevant space environments (e.g., star formation regions, evolved stars, circumstellar and interstellar medium) tells us that carbon components and silicates are key components of cosmic dust.

It is then instructive to perform laboratory experiments aimed at producing "analogues" of these cosmic compounds. Several techniques are used to obtain materials whose chemical and physical properties can simulate species present in space. The study is focused on the analysis of the optical properties versus intrinsic (e.g., chemistry, structure, morphology) characteristics of samples. Different complementary analytical techniques are combined with the aim of investigating the products of synthesis. Another primary goal of laboratory simulations is to trace material evolution versus processes similar to those active in space. The study of the reactivity of materials and of the efficiency of treatments provides quantitative information on how modifications, at the chemical and structural levels occur both in organic and in refractory materials. This goal is, therefore, achieved by iterative initially sequences in which samples, produced by different techniques, are analysed both as produced and after processing.

According to indications coming from astronomical observations, materials of primary interest are:

1) carbon compounds, in different forms (pure or hydrogenated, crystalline or amorphous);

2) silicates (crystalline or amorphous) of two main classes

- olivines $\left[\left(\mathrm{Mg}_{\mathrm{x}}, \mathrm{Fe}_{1-\mathrm{x}}\right)_{2} \mathrm{SiO}_{4}\right]$, where forsterite has $\mathrm{x}=1$ and fayalite $\mathrm{x}=0$,

- pyroxenes $\left[\left(\mathrm{Mg}_{\mathrm{x}}, \mathrm{Fe}_{1-\mathrm{x}}\right) \mathrm{SiO}_{3}\right]$, where enstatite has $\mathrm{x}=1$ and ferrosilite $\mathrm{x}=0$.

Several production techniques are used in the laboratory to synthesise samples useful to simulate cosmic compounds. Arc discharge between carbon/graphite electrodes in an inert (Ar, He) or $\mathrm{H}_{2}$ atmospheres (e.g., Colangeli et al. 1995), resistive heating of graphite rods coupled with molecular beam extraction (e.g., Schnaiter et al. 1996; 1998), IR laser pyrolysis of gas phase molecular species (e.g., Herlin et al., 1998) and quenching of hydrocarbon plasmas in vacuum (e.g., Sakata et al., 1984) are typical condensation techniques used for production of carbon-based material. High power laser bombardment of pure mineral targets in an $\mathrm{O}_{2}$ atmosphere (e.g., Brucato et al. 1999), as well as chemical reactions in gas phase (e.g., Nuth et al. 2000a), can be applied to obtain amorphous silicon-based materials of the desired chemical composition. Crystalline grains can be obtained by grinding pure terrestrial minerals. 
To test the sensitivity of laboratory analogues to cosmically relevant processes, several methods are used. When possible, laboratory experiments are performed by applying doses and rates comparable to those typical of different space environments. The summary in Table 2 reports a synthesis of experiments performed so far and a comparison of doses/rates achieved in the laboratory and estimated in space. All the examined mechanisms are expected to be effective in modifying most of the chemical and structural properties of materials.

\section{Table 2 (put here)}

The full characterisation of samples is obtained by a complement of techniques capable of demonstrating the relations between the nature of materials and their optical characteristics. Morphology and structure (shape, size and crystalline degree) are characterised by scanning electron microscopy (SEM), while the elemental composition can be identified by energy dispersive $\mathrm{X}$-ray (EDX) analysis. Further information on structure comes from X-ray diffraction and Raman spectroscopy. Spectroscopy is a diagnostic method to explore different properties of materials depending on the wavelength range used. Features in the UV-VIS and far IR ranges are generally associated with structural properties, while near-medium IR bands are diagnostic of molecular bond resonances. The detailed shapes and positions of bands are sensitive to morphology, chemical composition and crystalline degree. Moreover, different spectroscopic methods of analysis, such as extinction, absorption, scattering, reflectance and emission, allow us to examine different manifestations of the intrinsic natures of the considered samples.

Carbonaceous dust in the interstellar medium may show considerable diversity and may include amorphous carbon (AC), hydrogenated amorphous carbon (HAC), coal, soot, quenched-carbonaceous condensates (QCC), diamonds, and other compounds (e.g.Papoular et al. 1996, Henning \& Salama 1998, Ehrenfreund \& Charnley 2000 and reference therein). Figure 3 displays the basic chemical structure of carbonaceous interstellar dust in the diffuse interstellar medium (Pendleton \& Allamandola 2001). Observations indicate that a major fraction of the cosmic carbon in space is present in such a form.

\section{Figure 3 (put here)}

One of the most relevant signatures of interstellar dust is detected by observing the "extinction" of starlight caused by ISM material towards different sources in our Galaxy. The so-called "ultraviolet extinction bump" at $217.5 \mathrm{~nm}$ (e.g., Fitzpatrick \& Massa 1990) has an unambiguous attribution to carbon-bearing materials, but several candidate carriers (e.g., graphite, PAH's, amorphous carbon) fail to match the observational constraints and to account for the carbon budget, locked in solid form, available in space (Snow \& Witt 1995, 1996). 
Intense laboratory activity has focussed on the evolution of hydrogenated and hydrogen-free carbon materials subjected to different processes: thermal annealing (Mennella et al., 1995), UV irradiation (Mennella et al., 1996) and ion bombardment (Mennella et al., 1997). All experiments have demonstrated that carbon-based grains are extremely sensitive to processing and the efficiency of mechanisms in modifying organic material in space has been measured. For an example, the processing by UV photons of hydrogen-rich carbon dust produces the release of hydrogen and the progressive formation of small aromatic units (1-2 nm in size) forming the inner grain network. The optical manifestation of such change is the evolution from a featureless UV spectrum towards the appearance of a well defined band around $220 \mathrm{~nm}$, whose intensity grows as a function of applied UV photon doses.

The direct application of such a result to the astronomical context allows the possibility to simulate the "UV extinction band" observed in the ISM with a combination of UV processed carbon grain populations (see Figure 4). The approach based on such laboratory results provides a successful fit to observations and gives, for the first time, the opportunity to interpret the UV spectral characteristics in terms of carbon grain structural changes, perfectly compatible with the conditions actually expected in space. Moreover, the approach seems viable also in terms of elemental budget requirements, as about $50 \mathrm{ppm}$ of carbon (relative to hydrogen, $50 / 10^{6} \mathrm{C} / \mathrm{H}$ ) are needed, in the worst case, to match observations.

\section{Figure 4 (put here)}

Generally speaking, laboratory experiments confirm that carbon grains (also in space) may occur in two main forms: aliphatic structures are dominant in the presence of hydrogenation, while processes (such as thermal, UV, e-, ion mechanisms) tend to remove hydrogen and to favour the formation of aromatic domains inside the particles (Mennella et al. 2001).

The signature of polycyclic aromatic hydrocarbons is observed through their vibrational frequencies in the infrared between 3-15 $\mu \mathrm{m}$ (Tielens et al. 1999, Allamandola et al. 1999). Two decades of research show strong evidence that PAHs are present in the interstellar gas in many galactic and extragalactic regions. Up to 20 $\%$ of the carbon might be hidden in such aromatic species in the gas (Dwek et al. 1997). Spectroscopic studies in the laboratory have shown that free molecular PAHs can account for the overall appearance of the widespread interstellar infrared emission spectrum (Allamandola et al. 1999). Free individual PAH molecules, PAH clusters and particles comprised of aromatic (PAH) subunits are believed to be responsible for the overall interstellar emission spectrum. Figure 5 shows a comparison of an emission spectrum of a protoplanetary nebula and the Orion ionization ridge with different PAH mixtures. Nevertheless, it must be stated that no single PAH has yet been identified in the ISM.

\section{Figure 5 (put here)}


Silicates are clearly identified in infrared astronomical observations by the typical bands around 10 and $20 \mu \mathrm{m}$, due to Si-O stretching and O-Si-O bending modes, respectively. However, the scenario offered by spectral observations of silicates is complex. Crystalline $\mathrm{Mg}$-rich silicates are responsible for the sharp emission bands detected by ISO both in circumstellar environments (Malfait et al. 1998, Molster et al. 1999) and in comets (Crovisier et al. 1997). On the contrary, the broad bands at 10 and $20 \mu \mathrm{m}$ observed in the diffuse ISM (Whittet et al. 1997) and in dense clouds (Whittet et al. 1996) require the presence of silicates in an amorphous state. Therefore, we find spectral similarities and differences for silicates that reflect modifications induced in space according to the local environment.

With respect to silicates, laboratory experiments have been dedicated to the production of species with various relative metal (e.g., $\mathrm{Mg}, \mathrm{Fe}$ ) concentrations. Studies of their evolution due to thermal processing have produced a considerable amount of experimental data (e.g., Hallenbeck et al., 1998; Brucato et al., 1999; 2002; Fabian et al., 2000; Koike et al., 2001), which show that the synthetic materials are generally similar to both types of interstellar particles, but with non-negligible differences when analysed in detail. The two most relevant results of these experiments are that: a) the combination of time and temperature conditions required for the amorphous-to-crystalline transition are quite critical, when compared with the conditions available in space; b) the crystallisation of $\mathrm{Fe}$-rich olivine proceeds faster than for Mg-rich olivine, see Figure 6.

Astronomical observations seem to present a discrepancy with laboratory results, as Mg-rich crystalline silicates dominate in the astronomical spectra while Fe-rich olivine crystallizes faster in experiments. However, it should be considered that condensation models for a nebula with solar chemical composition show that almost all the Fe condenses as pure metal or alloy at temperatures above $600 \mathrm{~K}$ (Grossman 1972, Ebel 2000). Moreover, at least in the ISM, the temperature is too low for crystallisation to occur, so that the segregation between $\mathrm{Mg}$-rich silicates and pure metallic $\mathrm{Fe}$ should remain essentially unchanged when involved in planetary system formation. Coagulation processes in larger bodies inside the Solar System and thermal diffusion are necessary to include iron in silicates. Concerning the presence of signatures due to crystalline silicates in the spectra of comets, laboratory results pose a rough requirement of about $10^{6} \mathrm{yr}$ at $1000 \mathrm{~K}$ for the transition to occur. These conditions could not be achieved in the outer regions of the early Solar System. Mixing of materials from the inner Solar System region with the outer unevolved compounds must be invoked to justify the presence of crystalline silicates in comets, formed at the border of the protosolar nebula (Nuth et al. 2000b). Alternatively, mechanisms, other than pure thermal annealing, could be invoked to produce crystalline silicates in the presence of peculiar metastable conditions of the original materials (Molster et al., 1999). In this field much work still remains to be done in the laboratory to adequately support the interpretation of observations.

\section{Figure 6 (put here)}


Finally we must hold open the possibility that the apparent absence of crystalline silicates in the diffuse interstellar medium might be caused by an observational effect that has not yet been identified. This possibility emerges from observations of crystalline silicates in spectra of planetary nebulae, HII regions, and evolved stars (see Lequeux 2000 for review), and the absence of other indications of high temperature chemistry in cometary material. 


\section{Prebiotic molecules in protoplanetary disks}

\subsection{Overview of low-mass star formation}

For the development of life, solar-type stars have an important advantage. Since they evolve very slowly compared to more massive ones, there is at least one example of a solar-type star around which life had time to develop and to reach a high level of complexity. The 4.6 billion years of history our solar system enjoys is not shared by the bright stars that decorate our skies, most of which habe stable lives of less than 1 billion years. Even if high-mass stars are fundamental to the nucleosynthesis of heavy elements, we will in this section concentrate on studying the low-mass stars, which moreover form the most numerous stellar population.

A significant fraction of all stars in the galaxy are members of double or higher order multiple systems. One of the basic unsolved questions concerning the sun is whether it formed as part of a cluster, or in a more isolated event within a molecular cloud. Although some isotopic anomalies in meteorites suggest that formation in a cluster, which might have involved close physical and chemical interaction with supernova debris, is more likely, theories of solar type star formation have predominantly assumed the isolated scenario described below.

The formation of stars occurs through the gravitational collapse of individual galactic molecular cloud cores within the dense molecular clouds such as described in section 2. As soon as the collapse has started, one can roughly consider that there are two main phases. In the protostellar phase, the central protostar strongly embedded in its parent cloud is accreting surrounding material and is ejecting in the meantime a powerful bipolar outflow which allows it to regulate the angular momentum resulting from the original contraction of the rotating parent cloud. After about one million years, the surrounding cloud has dissipated sufficiently and the young stellar object becomes visible; this is the T-Tauri phase (from the name of the first discovered object). The star has a mass very close to its final value, and is surrounded by a disk of dust and gas. T-Tauri stars are precursors of solar-type stars (mass range $0.5-2$ solar masses). After about 50 million years more, these objects finally begin their life as "adult" stars by reaching the Main-Sequence of the temperature-luminosity diagram. In the meantime, the surrounding protoplanetary disk has dissipated and planets may have formed in orbit around the stars. The surrounding residual dust disk, called a "debris disk", is now optically thin in the near-infrared (NIR). In such disks, grains originate from the collisions of larger bodies such as planetesimals, the lifetime of dust particles being very short compared to the disk lifetime (Lagrange et al. 2000 ). The prototype of such an object is the famous $\beta$-Pic disk (Vidal-Madjar et al. 1998).

Figure 7 (from van Dishoeck \& Blake 1998) summarizes the important steps leading to planetary formation with emphasis given to the chemical evolution of the gas phase during the formation of a low-mass star. This figure describes the observed molecules depending on the physical conditions that evolve with time. For a more complete review of stellar formation, the reader can refer to Dutrey (1999).

Figure 7 (put here) 


\subsection{From protoplanetary disks to planets}

It is during the protoplanetary disk phase that planetary formation is thought to begin. Many of these disks are indeed massive enough (0.1- 0.001 solar mass) to provide the reservoir of matter necessary to form planetary systems. Heated by the central $\mathrm{T}$ Tauri star, these disks are cold, with typical temperatures within the range $\sim 10-100$ $\mathrm{K}$, and they therefore radiate at millimeter and submillimeter wavelengths. Only the central 10-30 astronomical units (AU), also heated by viscous accretion (D'Alessio et al. 1999), are significantly hotter. Millimeter spectroscopic interferometric observations ( $\mathrm{CO}$ rotational lines) confirm that these objects are in Keplerian rotation around their central star (Koerner et al., 1993, Dutrey et al., 1994). Both millimeter interferometric $\mathrm{CO}$ maps and optical images of dust disks scattering light from their central star also revealed that many disks are large, with radii of $\sim 100-1000 \mathrm{AU}$. The gas and dust are remnant material from the molecular cloud, and most of the mass is still in the gas phase (a gas-to-dust ratio of 100 is usually assumed). The molecular content is poorly known; $\mathrm{H}_{2}$ is, however, the most abundant molecule, while $\mathrm{CO}$ remains the most abundant species containing carbon. The chemical conditions change with the disk radius. Studying the molecular disk content close to the star is, however, out of the scope of current telescopes due to lack of adequate angular resolution. Recent models of disks irradiated by stellar X-ray emission also show that $\mathrm{X}$-rays can significantly affect the disk surface and can even penetrate deep inside the disk (Najita et al., 2001), with important chemical implications.

Dutrey et al. $(1997,2000)$ summarizes the molecules detected in the protoplanetary disk surrounding DM Tau, a T-Tauri star of 0.5 solar mass. This corresponds to the deepest survey performed so far. Because of sensitivity limitations, the observed molecules only trace the outer disk chemistry (radius $>50 \mathrm{AU}$ ). Simple organic molecules like $\mathrm{H}_{2} \mathrm{CO}$ or $\mathrm{HCN}$ have been detected, suggesting that even more complex molecules may exist. The molecules that are currently detected correspond to the most abundant species found in dark clouds (abundances in TMC-1 are given in col.2), but all the molecules appear depleted with respect to TMC-1 abundances (col.5). The outer regions of these disks are very cold and dense and molecules are expected to condense on dust grains. Note that such molecular depletions do imply the possible depletion of $\mathrm{H}_{2}$ with respect to the dust, because of the much greater volatility of $\mathrm{H}_{2}$.

In conclusion, due to a lack of sensitivity and angular resolution, the evolution of disks toward planetary formation is poorly constrained today, and only a few organic molecules are observed in protoplanetary disks.

Table 3 (put here) 


\section{Planets}

When we had only our own system to study, the widely accepted model for its formation began with the collapse of a fragment of an interstellar cloud into a disk (see section 4) called the solar nebula, dominated by a central condensation that became the sun. Accretion of dust grains in the disk led to the formation of planetesimals that ultimately collided with one another to form the planets. Beyond the distance from the sun at which ice could first condense, often referred to as the "snowline", this plentiful raw material allowed the formation of solid objects with masses ten times the Earth's or more, sufficient to enable the gravitational collapse of surrounding solar nebula gas. The existence of a snowline thus explained the location of the giant planets in the outer part of the solar system. Analogues of rocky planetesimals formed in the inner nebula remain today as asteroids and meteorites, while in the outer solar system, the icy planetesimals are represented by comets, centaurs and Kuiper Belt Objects.

This brief outline omits an extremely complex series of interactions that are still poorly understood in detail. But the greatest current challenge to the model comes from the planets discovered around other stars during the last five years. These new planets are all giants almost all are more massive than Jupiter. Their most surprising characteristic is their proximity to their stars: instead of orbiting outside the "snowline", all of the 70-odd giant planets discovered to date circle their stars at distances less than 4.5 AU (Marcy et al. 2000, http://exoplanets.org, Queloz 2000). Nearly 30 of them have orbits smaller than Mercury's! Within 0.07 AU, the orbits are nearly circular, but at greater distances, another major surprise occurs: their eccentricities increase to values as high as 0.7 . Such highly elliptical orbits are only occupied by comets in our solar system.

What are we to make of these glaring differences with our own planetary system? Could it be that our configuration of planets is extremely rare, perhaps even unique? If so, prospects for finding communicative aliens elsewhere in the galaxy are dim indeed. Models that explain the configurations of these newly-discovered systems invoke tidal interaction between the giant planets and the surrounding disk of gas and dust that could cause the planets to migrate inwards toward their stars from their original, distant positions (Lin \& Papaloizou 1986, Lin \& Ida 1997). Such migrations follow the inward movement of the disks themselves, suggesting that any Earth-like planets in such systems would be swept into the central stars by the migrating giants.

There is still a possibility that small planets could be captured into stable positions in the giant planets' orbits, much Jupiter has captured asteroid-sized bodies in the stable Lagrangian points 60 degrees ahead of and behind the position of the planet itself. One could also invoke the change in scale appropriate to such giant planets to suggest the presence of giant satellites that might possess Earth-like characteristics (Owen 2000). Note that Jupiter's satellite Europa is currently viewed as a potential carrier of life, because of evidence suggesting the presence of a subsurface liquid water ocean, heated by tidal interaction with Jupiter. In either of these extreme cases, one must assume that the giant planet ceases its migration when the semi-major axis of its orbit is about $1 \mathrm{AU}$, and indeed a few such planets have been discovered among the dozens now known to exist. 
But it is premature to invoke such speculative scenarios when the data on these new planetary systems are so incomplete. It is important to note that giant planets close to their stars are by far the easiest planets to discover with presently available methods and consequently the sample of planetary systems from which we are trying to glean insight distorted by observational bias. The first extra solar planet was discovered in 1995 (Mayor \& Queloz, 1995), so we must expect to wait several more years to have reliable detections of planets in orbits with 12-year periods and low eccentricities. A system with such a planet would be the first one that resembles our own. The detection of Earth size planets in such systems will have to await the launch of dedicated orbiting telescopes. 


\section{Comets in brief}

The nucleus of a comet is a conglomeration of ices and grains of organic compounds and silicates. This composition leads immediately to the perennial question about the ancestry of comets: just how closely do these icy objects resemble a simple assemblage of interstellar grains ? The ratio of dust and ice varies considerably from comet to comet, allowing description of the nucleus to range from an "icy dirtball" to a "dirty iceball". Traditionally, comet nuclei have been thought of as the building blocks of the $\sim 10$ Earth mass solid cores that initiated the formation of the giant planets. Recently, however, it has become apparent that, at least in the case of Jupiter, those building blocks were solar composition icy planetesimals (SCIPs) (Owen et al. 1999). The high volatile content of SCIPs makes them considerably more primitive than the nitrogen- and argon deficient comets that we know. The origin of the SCIPs is still under discussion (Owen et al. 1999, Owen 2000, Gauthier et al. 2001a,b). In contrast, it is widely assumed that the comets were formed in the outer solar nebula, beyond the orbit of Jupiter.

Early in the history of the solar system, Jupiter and the other giant planets perturbed the orbits of the comets in their vicinities. Some of these comets then collide with the sun, some with the terrestrial or giant planets, some were ejected out of the solar system, a fraction coming from the whole Jupiter-Neptune zone built the Oort cloud, whereas Kuiper-Belt objects were probably formed in the belt itself (Weissman, 1999). The composition of comets has been recently reviewed (Altwegg et al. 1999, Crovisier \& Bockelee-Morvan 1999, Irvine et al. 2000, Ehrenfreund \& Charnley 2000 , Charnley et al. 2002), as well as various aspects of their possible role in the origin and evolution of life (Thomas et al. 1997). A general presentation of comets can be found in Crovisier \& Encrenaz (2000) and a detailed account of many aspects in Festou (1993a,b) and in Newburn et al. (1991).

Cometary nuclei nowadays mostly form in two reservoirs, the spheroidal Oort cloud, up to $10^{4}-10^{5} \mathrm{AU}$ from the sun, and the annular Edgeworth-Kuiper Belt beyond Neptune, 30-100 AU from the sun. Their estimated total mass is between 1 and 250 Earth masses and between 0.1 and 1 Earth mass, respectively, for bodies $0.5-500 \mathrm{~km}$ in size (the Halley nucleus, for example, was $8 \times 8 \times 16 \mathrm{~km}$ ). More than 100 new comets are discovered each year, when the highly elliptic orbits of the Oort cloud comets bring them into the zone of the planets. Closer than $10 \mathrm{AU}$ to the sun, comets become active and release volatiles, first mainly $\mathrm{CO}$ and then, within $4 \mathrm{AU}$, mainly $\mathrm{H}_{2} \mathrm{O}$, which drag other species and dust grains. The largest grains spread along the orbit and contribute to the interplanetary dust responsible for meteors showers. We use in the following the short names Halley, Hyakutake and Hale-Bopp, but the correct astronomical names of these comets are 1P/Halley, C/1996 B2 Hyakutake and - C/1995 O1 Hale-Bopp. 


\section{Comets in brief}

The nucleus of a comet is a conglomeration of ices and grains of organic compounds and silicates. This composition leads immediately to the perennial question about the ancestry of comets: just how closely do these icy objects resemble a simple assemblage of interstellar grains? The ratio of dust and ice varies considerably from comet to comet, allowing description of the nucleus to range from an "icy dirtball" to a "dirty iceball". Traditionally, comet nuclei have been thought of as the building blocks of the $\sim 10$ Earth mass solid cores that initiated the formation of the giant planets. Recently, however, it has become apparent that, at least in the case of Jupiter, those building blocks were solar composition icy planetesimals (SCIPs) (Owen et al. 1999). The high volatile content of SCIPs makes them considerably more primitive than the nitrogen- and argon deficient comets that we know. The origin of the SCIPs is still under discussion (Owen et al. 1999, Owen 2000, Gauthier et al. 2001a,b). In contrast, it is widely assumed that the comets were formed in the outer solar nebula, beyond the orbit of Jupiter.

Early in the history of the solar system, Jupiter and the other giant planets perturbed the orbits of the comets in their vicinities. Some of these comets then collide with the sun, some with the terrestrial or giant planets, some were ejected out of the solar system, a fraction coming from the whole Jupiter-Neptune zone built the Oort cloud, whereas Kuiper-Belt objects were probably formed in the belt itself (Weissman, 1999). The composition of comets has been recently reviewed (Altwegg et al. 1999, Crovisier \& Bockelee-Morvan 1999, Irvine et al. 2000, Ehrenfreund \& Charnley 2000 , Charnley et al. 2002), as well as various aspects of their possible role in the origin and evolution of life (Thomas et al. 1997). A general presentation of comets can be found in Crovisier \& Encrenaz (2000) and a detailed account of many aspects in Festou (1993a,b) and in Newburn et al. (1991).

Cometary nuclei nowadays mostly form in two reservoirs, the spheroidal Oort cloud, up to $10^{4}-10^{5} \mathrm{AU}$ from the sun, and the annular Edgeworth-Kuiper Belt beyond Neptune, 30-100 AU from the sun. Their estimated total mass is between 1 and 250 Earth masses and between 0.1 and 1 Earth mass, respectively, for bodies $0.5-500 \mathrm{~km}$ in size (the Halley nucleus, for example, was $8 \times 8 \times 16 \mathrm{~km}$ ). More than 100 new comets are discovered each year, when the highly elliptic orbits of the Oort cloud comets bring them into the zone of the planets. Closer than $\sim 10 \mathrm{AU}$ to the sun, comets become active and release volatiles, first mainly $\mathrm{CO}$ and then, within $4 \mathrm{AU}$, mainly $\mathrm{H}_{2} \mathrm{O}$, which drag other species and dust grains. The largest grains spread along the orbit and contribute to the interplanetary dust responsible for meteors showers. We use in the following the short names Halley, Hyakutake and Hale-Bopp, but the correct astronomical names of these comets are 1P/Halley, C/1996 B2 Hyakutake and C/1995 O1 Hale-Bopp. 


\subsection{Composition of cometary matter}

Comets contain, within a factor of ten, roughly the same mass of ices and of dust. Some comets appear richer or poorer in dust, which seems to be due to an initial composition difference (A'Hearn et al. 1995). Note that the dust mass is difficult to evaluate precisely, due to the large range of particle sizes and the possible differences in particle size distribution from one comet to another.

The knowledge of the chemical composition of cometary ices is now mainly based on measurements by millimeter wave or infrared spectroscopy of the molecules in the coma, which sublimated from the nucleus (e.g. Irvine et al. 2000, Bockelée-Morvan et al. 1997, 1998, Despois 1997/99, Crovisier 1997/99). The derivation of the abundances in the nucleus from these observations normally assumes that the molecules come directly from the nucleus (no carrier), and any chemical process in the coma other than photochemistry are usually neglected; this has been questioned for some of the species ( $\mathrm{CO}, \mathrm{H}_{2} \mathrm{CO}, \mathrm{HNC}$; see below). In the future cometary observations may have to be interpreted in the context of a nucleus whose ices are stratified in density, temperature and porosity (Prialnik \& Podolak 1999).

\section{Table 4 (put here)}

Cometary ices are predominantly (more than $50 \%$ ) water ice; but by now more than 25 other small molecules have been identified. Their abundances in percent relative to water molecules are listed in Table 4, and range from 20-30 \% (for CO) down to 0.01 $\%$, which is at present the lowest abundance detected. The inventory of cometary species is certainly not yet complete in the range $0.01-1 \%$. Many small reactive molecules important in prebiotic aqueous chemistry are detected: $\mathrm{H}_{2} \mathrm{O}, \mathrm{CO}_{2}, \mathrm{H}_{2} \mathrm{CO}$, $\mathrm{NH}_{3}, \mathrm{HCN}, \mathrm{CH}_{3} \mathrm{CN}$, $\mathrm{HNCO}$ and $\mathrm{H}_{2} \mathrm{~S}$. But other species, less often considered as components of the "primitive soup", are also present in noticeable amounts, and may play a role in aqueous or anhydrous synthesis of more complex species, e.g. $\mathrm{CH}_{3} \mathrm{OH}$ (1-5\%), $\mathrm{C}_{2} \mathrm{H}_{2}(1 \%)$ and $\mathrm{CO}$ (up to $30 \%$ ). The most complex species known at present are: $\mathrm{CH}_{3} \mathrm{CHO}, \mathrm{HCOOCH}_{3}$, and $\mathrm{NH}_{2} \mathrm{CHO}$ - which bears some resemblance to glycine, $\mathrm{NH}_{2} \mathrm{CH}_{2} \mathrm{COOH}$. Glycine, the simplest amino acid, has itself not yet been detected, and the present upper detection limit of $0.5 \%$ is not very significant compared to other species.

All comets are expected to contain more or less the same species, but relative abundances may differ : from 1 to $5 \%$ for $\mathrm{CH}_{3} \mathrm{OH}$, from less than $1 \%$ up to $30 \%$ for $\mathrm{CO}$. The variation in $\mathrm{CH}_{3} \mathrm{OH}$ may reflect formation in different zones of the protosolar nebula (Mumma et al. 2001). On the other hand, the high volatility of $\mathrm{CO}$ is probably the main reason for its variation from comet to comet, which may be linked again to the comet formation zone, but also to the aging of comets and to the distance from the sun at which the active comet is observed. Such a differential sublimation does not seem to affect the relative production rate of other, less volatile, species, with two exceptions (in addition to $\mathrm{CO}$ ): $\mathrm{H}_{2} \mathrm{CO}$ and $\mathrm{HNC}$ (Biver 1997/99). Release of $\mathrm{H}_{2} \mathrm{CO}$ from grains, and especially those containing its - polymer (polyoxymethylene, POM), has been proposed (Huebner 1987, Cottin et al. 2001). For HNC, interconversion between $\mathrm{HNC}$ and $\mathrm{HCN}$ through chemical reactions has been proposed (Irvine et al. 1998b, Rodgers \& Charnley 1998, 2001). 
The chemical composition of cometary grains has been analyzed in situ in comet 1P/Halley by mass spectrometers on board the Vega 1 and 2 and Giotto spacecraft (Fomenkova 1999). Approximately 5000 grains have been collected, with masses from $5.10^{-17}$ to $5.10^{-12} \mathrm{~g}$ (and sizes from a few hundreths of $\mu \mathrm{m}$ to a few $\mu \mathrm{m}$ ); the total analyzed mass was thus only a few nanograms. Grains contain carbonaceous matter and silicates. There are three types of grains depending on their relative abundances: "CHON grains", containing mainly $\mathrm{C}, \mathrm{H}, \mathrm{O}, \mathrm{N}$ atoms; rocky grains, containing rock-forming elements : $\mathrm{Si}, \mathrm{Al}, \ldots$; and mixed grains. One possibility for these is a carbonaceous matrix containing small mineral particles, as observed in Interplanetary Dust Particles.

The carbon rich grains are apparently of various types: i) pure carbon particles; ii) PAHs (polycyclic aromatic hydrocarbons) iii) branched aliphatic hydrocarbons iv) carbon-oxygen or carbon-nitrogen polymers and v) more complex compounds containing all four $\mathrm{C}, \mathrm{H}, \mathrm{O}$ and $\mathrm{N}$ atoms (heteropolymers or complex organic molecules have been proposed). A more specific list of organic compounds in the grains has been proposed by Kissel et al. (1997) but it remains very speculative due to the limited resolution of the mass spectrometers, which flew through Halley's coma.

The detection of a PAH, phenantrene, has also been announced in Halley through UV spectroscopy (Moreels et al. 1994); however no PAHs have been seen in the ISO IR spectra of C/1995 O1 Hale-Bopp (Crovisier 1997/99), which may be due to a larger heliocentric distance.

The nature of the silicate particles has been partly revealed also by infrared observations. Laboratory spectra of olivines $\left(\mathrm{XSiO}_{4}\right)$ and pyroxenes $\left(\mathrm{X}_{2} \mathrm{SiO}_{3}\right)$, either in crystalline or amorphous form, with $\mathrm{X}$ being $\mathrm{Fe}, \mathrm{Mg}$, etc. in various proportions, and of carbonaceous particles are combined to match cometary spectra (see section 3). ISO observations (Crovisier 1997/99) led to the identification of forsterite, a crystalline $\mathrm{Mg}$-rich olivine. The pyroxene/olivine ratio appears ten times higher than in carbonaceous chondrites (primitive meteorites, supposed to come from asteroids, whose composition is the closest to comets). The presence of crystalline silicates is a major puzzle when trying to link cometary and interstellar matter.

\subsection{Comets and the origin of life}

Made of ices and dust (carbonaceous and silicate micron-sized grains), comets are rich in water and carbon, two key constituents of terrestrial life. Moreover, as realized by Oro already in 1961 (Oro,1961), they can bring to Earth reactive C-bearing molecules which might assemble to form amino-acids, purine and pyrimidine bases and sugars, i.e. the basic building blocks of our genetic material, DNA, RNA and proteins. The importance of this fact was greatly increased by those adopting models of the primitive atmosphere of the Earth that assume was a $\mathrm{CO}_{2}-\mathrm{H}_{2} \mathrm{O}-\mathrm{N}_{2}$ mixture, much less prone to produce complex organic molecules than the mixtures of reducing gases used in the original Miller-Urey experiments. Extraterrestrial delivery of complex molecules is then a very competitive source of organic matter, as shown in the pioneering work of Chyba \& Sagan $(1992,1997)$, see section 8. 
The importance of comets for the origin of life on Earth has also been strongly advocated by other authors (e.g. Delsemme 1992,2000; Greenberg 1992, 1996). Could comets deliver organics made in the ISM to the surfaces of rocky planets? This is a contentious subject at the moment, with advocates of preservation of pristine interstellar material arrayed against those who think considerable reprocessing and mixing occurred in the solar nebula. The most common argument for the preservation of interstellar material in comets comes from a comparison of their molecular composition, as revealed by studies of their comae, and the list of molecules found in interstellar clouds (e.g., Crovisier \& Bockelee-Morvan 1999, Bockeleé-Morvan et al. 2000). Additional support can be derived from measurements of $\mathrm{D} / \mathrm{H}$, see sections 9 and 10. In the one comet where it was measured (Hale-Bopp, by Meier et al. 1998) $\mathrm{D} / \mathrm{H}$ in $\mathrm{HCN}$ is 7 times higher than the value found in $\mathrm{H}_{2} \mathrm{O}$. This is the factor expected for deuterium exchange via ion-molecule reactions at a temperature of $30 \pm$ $5 \mathrm{~K}$ (Millar et al. 1989). Measurements of the ratio of ortho to para hydrogen in cometary $\mathrm{H}_{2} \mathrm{O}$ and $\mathrm{NH}_{2}$ yield temperatures of $23 \pm 5$ and $28 \pm 3$ respectively (Crovisier 1998, Kawakita et al. 2001). This agreement suggests that interstellar chemistry has been preserved in the cometary formation process. Another indicator of such preservation is provided by the high value of $\mathrm{D} / \mathrm{H}$ and ${ }^{15} \mathrm{~N} /{ }^{14} \mathrm{~N}$ found in cluster IDPs (Messenger 2001). These are the most fragile of the Interplanetary Dust Particles and thus the ones most commonly assumed to come from comets. These anomalous high isotope ratios require an interstellar source and they clearly have not been allowed to re-equilibrate with hydrogen gas in the solar nebula.

Some of these high isotope ratios found in organic material surrounding crystalline silicates suggest that these silicates were also brought in from the ISM (Keller et al. 2000). The existence of crystalline silicates in comets- as revealed by cometary IR spectra - and their apparent absence from the ISM, has been invoked as an argument for mixing of inner nebula material to the outer nebula (e.g., Mousis et al. 2002). The argument is that crystalline silicates can only be formed near stars where temperatures above $1000 \mathrm{~K}$ exist, see section 2 . Since they are not seen in the ISM, their presence in comets requires formation near the sun followed by transport to the outer nebula and incorporation in forming comets. However, as stated in section 2 , the apparent absence of crystalline silicates from the ISM is a puzzle, in that they are evidently delivered there (Lequeux 2000). Furthermore, the observed chemical composition of comets - retaining the mix of fully oxidized and fully reduced carbon compounds in rougly interstellar proportions, argues against extensive mixing with material exposed to high temperatures. Nevertheless, we still miss a clear direct connection between the composition of comets and the composition found in interstellar clouds, so that testing the link between comets and the ISM remains an important research topic. The determination of $\mathrm{D} / \mathrm{H}$ in other cometary molecules besides $\mathrm{H}_{2} \mathrm{O}$ and $\mathrm{HCN}$ may provide a definitive result. In section 8 and 10 the conditions for extraterrestrial delivery are further elaborated. 


\section{Chemical composition and classification of the meteorites}

Meteorites are usually divided into three main groups, the iron meteorites, the stony irons and the stones. The iron meteorites are composed of at least $90 \%$ metal, mainly iron and nickel. The stony iron meteorites are further divided into pallasites, which mainly comprise olivine crystals embedded in a metallic matrix, and mesosiderites, also made up of silicates and metal but more finely grained and thus intimately mixed. The stone meteorites consist predominantly of silicates and are divided into chondrites and achondrites due to the presence or absence of infra-millimetric spheres termed chondrules. Three groups are distinguished among the chondrites according to their chemical composition (ordinary, enstatite and carbonaceous). The ordinary chondrites correspond to the most abundant group and are further divided into three sub-groups on the basis of total iron content and iron content in the olivines and pyroxenes (H-chondrites for high total iron, $\mathrm{L}$ - for low total iron and LL- for low total iron and low metal). Enstatite chondrites are characterized by a high iron content but the latter contains virtually no silicates.

Carbonaceous chondrites include several groups designated according to the initial of the type specimen (e.g., CI after Ivuna, CM after Mighei) and are all characterized by a black color. CI chondrites exhibit the same elemental composition as the sun with the exception of some gas-forming elements such as $\mathrm{He}, \mathrm{H}, \mathrm{O}, \mathrm{N}$ and $\mathrm{C}$ and are thus considered as the most primitive rocky bodies of the solar system apart from comets. Carbonaceous chondrites have been shown to contain organic substances, which will be described below in more detail. Achondrites show a magmatic texture and are considered to be derived from more primitive material by a melting process, in contrast to the chondrites, which have retained their original composition. The most abundant achondrites (eucrites, howardites and diogenites) resemble terrestrial basalts. Aubrites are also referred to as enstatite achondrites and are supposed to be derived from enstatite chondrites through melting and differentiation processes. Ureilites are characterized by a high carbon content $(\sim 2 \%)$ and by the occurrence of diamonds and are thus assumed to have originated from carbonaceous chondrites. SNC meteorites, namely shergottites, nakhlites and chassignites, contain sodium-rich feldspar and in some cases minerals with crystallization water suggesting a formation under more oxidizing conditions. Their young age (only a few hundred million years with respect to 4.6 billion years for chondrites) points to a relatively large parent body that underwent magmatic activity after its formation, and the elemental and isotopic composition of the noble gases and nitrogen trapped in these rocks revealed a Martian origin for the SNC meteorites. 


\subsection{Macromolecular organic matter in meteorites}

Carbonaceous chondrites are known to contain a substantial amount of $\mathrm{C}$, up to $3 \%$. The major part of this carbon corresponds to a macromolecular organic fraction (Hayes, 1967). This insoluble organic matter (IOM) is usually isolated from the bulk meteorite as the insoluble residue that is left after solvent extractions and drastic acid treatments using $\mathrm{HF}$ and $\mathrm{HCl}$, which aim at removing the soluble organic compounds and the bulk of the minerals, respectively.

A number of analytical tools can be considered for the study of such refractory organic material, including spectroscopic methods such as Fourier Transform Infrared (Hayatsu et al., 1977; Khare et al., 1990; Ehrenfreund et al., 1991, 1992b; Salisbury et al., 1991; Murae, 1994), Raman (Strazzulla et al., 2001), solid state ${ }^{13}$ C NMR (Nuclear Magnetic Resonance) (Cronin et al., 1987; Gardinier et al., 2000; Pizarello et al., 2001), XANES (X-ray Absorption Near-Edge Structure) (Cody et al., 1999; Flynn et al., 1999) and EPR (Electron Paramagnetic Resonance) (Duchesne et al., 1964; Schulz and Elofson, 1965; Binet et al., submitted). Infrared spectroscopy pointed to pronounced similarities between carbonaceous grains in the interstellar medium and IOM in carbonaceous chondrites, the main two characteristic features being (1) an equivalent intensity of the asymmetric stretching mode absorptions for $\mathrm{CH}_{3}\left(2960 \mathrm{~cm}^{-1}\right)$ and $\mathrm{CH}_{2}\left(2920 \mathrm{~cm}^{-1}\right)$ and (2) the lack of aromatic stretching mode absorption $\left(3040 \mathrm{~cm}^{-1}\right)$. Micro-Raman investigation in the Orgueil meteorite revealed that the amorphous carbon is homogenous and suggested that this material has not been exposed to significant irradiation (Strazzulla et al. 2001).

Solid state ${ }^{13} \mathrm{C}$ NMR is a powerful technique to determine the nature and environment of carbons in macromolecular materials. Since the pioneering study carried out by Cronin in 1987, it has been recently applied to the IOM of the meteorites of Orgueil, Murchison and Tagish Lake showing their highly aromatic character (Gardinier et al., 2000; Pizzarello et al., 2001). This method also revealed a high level of branching of the aliphatic chains and showed that the aromatic units are highly substituted, especially in Murchison, see Figure 8. These results show that the IOM of these meteorites did not suffer a marked thermal maturation during the late hydrothermalism on the parent body. Its synthesis involved a statistical combination of all the possible bonds between $-\mathrm{CH}_{3},-\mathrm{CH}_{2}$ and $-\mathrm{CH}$ radicals, producing both the aliphatic and aromatic moieties. This model explains not only the chemical features of the chondritic IOM but also the typical FTIR signature of the interstellar medium (Gardinier et al., 2000).

\section{Figure 8 (put here)}

C-XANES spectra of the IOMs of Murchison, ALH 84001 and Nakhla show intense absorptions at $285 \mathrm{eV}$ assigned to aromatic organics as well as at $288.6 \mathrm{eV}$, along with a weaker peak at $286.8 \mathrm{eV}$ (Cody et al., 1999; Flynn et al., 1999). N-XANES showed similar spectra for Orgueil and Murchison IOMs, with strong absorptions due to pyridine, pyridone and pyrrole rings whereas significant differences are observed between these two meteorites when the S-XANES spectra are considered (Derenne et al., in prep.). 
Indeed, firstly a peak is observed at low energy in the case of Murchison due to the survival of a significant contribution of pentlandite (an iron-nickel mineral sulfide) upon IOM isolation. Moreover, when the organic sulfur species are considered, their relative abundances calculated after deconvolution of the spectra are different in Orgueil and Murchison. Indeed, (i) a significant contribution of oxidized species (sulfoxides, sulfones) is observed in Orgueil, whereas they are virtually absent in Murchison and (ii) the aliphatic sulfides to thiophenes ratio is much higher in Murchison than in Orgueil (Derenne et al., in prep.).

These differences may be related to different intensities in the hydrothermal processes undergone on the parent body. Based on detailed mineralogical studies, it is generally accepted that carbonaceous chondrites underwent hydrothermalism on the parent body (Bunch \& Chang 1980). The nature of the organic free radicals in chondritic IOM has been revisited recently using EPR and ENDOR (Electron Nuclear Double Resonance) (Binet et al., submitted). This study confirmed the occurrence of a relatively high concentration of radicals $\left(>10^{18} \mathrm{~g}^{-1}\right)$ in Orgueil and Murchison and showed that these radicals (i) exhibit a moderate level of oxygenation and (ii) are not randomly distributed in the IOM. Indeed, areas with much higher local concentrations of radicals (about 3-4 $10^{19}$ spin. $\mathrm{g}^{-1}$ ) are evidenced (Binet et al., submitted). These radical-rich regions can be viewed as pristine parts of the $\mathrm{OM}$ which would have survived the hydrothermal process on the parent body.

Chemical and thermal degradation provide information at the molecular level. Ozonolysis of Orgueil IOM released a number of benzenepolycarboxylic acids pointing to a low level of condensation for the organic matter (Bitz \& Nagy 1966). Such products were also detected in the alkali soluble fraction of Murchison IOM along with alkylbenzenes and phenolic compounds (Studier et al. 1972) and, after oxidation with nitric acid, various other aromatic acids such as naphthalene acids and pyridine acids (Hayatsu et al., 1977). Chemical degradation of Murchison IOM using $\mathrm{CF}_{3} \mathrm{COOH}, \mathrm{Cr}_{2} \mathrm{O}_{7}$ and alkaline $\mathrm{CuO}$ oxidation also yielded a number of aromatic compounds including naphthalene, phenanthrene, anthracene, indane, pyrrole and benzothiophene, but some aliphatic hydrocarbons and acids were also shown to be released (Hayatsu et al., 1977, 1980). The first pyrolysis of Murchison IOM, performed as a direct heating in the mass spectrometer source, released aromatic hydrocarbons containing up to three rings (Studier et al., 1972). Pyrolysis at various temperatures, ranging between $150^{\circ} \mathrm{C}$ and $600^{\circ} \mathrm{C}$, showed that most of the pyrolysis products are generated from Murchison and Orgueil between 300 and $450{ }^{\circ} \mathrm{C}$ (Levy et al., 1973; Bandurski \& Nagy, 1976). Alkylthiophenes and benzothiophene were also reported in these pyrolysates, but is was noted that they may be formed through cyclisation and aromatisation of other compounds upon pyrolysis. Aliphatic compounds, i. e. alkanes and alkenes up to $\mathrm{C}_{8}$, have been shown to be present in these pyrolysates (Bandurski \& Nagy, 1976; Hayatsu et al., 1977). Acetonitrile and acrylonitrile were identified and considered as possibly derived from proteins or amino acids (Bandurski \& Nagy, 1976). High temperature pyrolysis $\left(600{ }^{\circ} \mathrm{C}\right)$ of Murchison IOM was performed as an attempt to release fullerenes and led to the detection of numerous high molecular weight PAHs including coronene (de Vries et al., 1993). Examination of the IOM from several CM2 chondrites from Antarctica by pyrolysis/gas chromatography/mass spectrometry showed that these chondrites were more or less affected by aqueous alteration and thermal metamorphism (Komiya et al., 1993). 
Similar products as those obtained from the above mentioned carbonaceous chondrites were released upon pyrolysis/GC/MS of the IOM of Nakhla (Jull et al., 1999). Hydrous pyrolysis, i. e. pyrolysis in the presence of water, has been performed on Murchison, Cold Bokkeveld (two CM chondrites) and Orgueil (a CI chondrite) IOM (Sephton et al., 1998, 1999). The samples have been heated at $320^{\circ} \mathrm{C}$ for $72 \mathrm{~h}$ in a sealed vessel. In addition to aromatic hydrocarbons, phenolics have been detected pointing to the occurrence of ether bridges between the aromatic units (Sephton et al., 1998). Moreover, these results suggest a higher extent of condensation of the aromatic units along with a higher contribution of ether linkages in the $\mathrm{CM}$ chondrites than in the CI one, which may be related to the more extensive aqueous alteration undergone by Orgueil (Sephton et al., 1999, 2000).

Stepwise combustion of the IOM of Murchison and Orgueil revealed isotopic heterogeneities in $\mathrm{C}, \mathrm{N}$ and $\mathrm{H}$ (Halbout et al., 1980; Kerridge et al., 1987). This has been recently confirmed in an extensive study on one $\mathrm{CI}$ chondrite (Orgueil), three CM chondrites (Murchison, Murray and Cold Bokkeveld), one CR chondrite (Renazzo), five ordinary chondrites including Semarkona and three enstatite chondrites (Alexander 1998). Based on the $\mathrm{N}$ isotope release profile, it appears that all the chondrites have accreted similar organic material, which can still be observed in Renazzo and Semarkona (Alexander 1998). However, the origin of the chondritic IOM is not yet well understood and no model is so far able to explain its observed structural and isotopic diversity.

Isotope composition can now also be obtained at a molecular level thanks to pyrolysis-GC-irMS which allows the determination of the C-isotope composition of the individual pyrolysis products. The latter was recently performed on the IOM of Nakhla so as to distinguish indigenous OM from terrestrial contamination (Jull et al., 1999). This technique allows the determination of the isotopic and chemical features of the IOM isolated from small amounts of meteorites (Sephton \& Gilmour, 2001).

\subsection{Volatile Organic Compounds in meteorites}

The classes of soluble meteoritic organic compounds that have familiar biochemical counterparts include amino acids, fatty acids, purines, pyrimidines and sugars (Botta et al. 2001). Moreover, alcohols, aldehydes, amides, amines, mono- and di-carboxylic acids, aliphatic and aromatic hydrocarbons, polar compounds such as heterocyclic aromatics, hydroxy acids, ketones, phosphonic and sulfonic acids, sulfides, ethers and simple sugars have also been reported (Cooper et al., 2001, Cronin et al., 1988, Botta \& Bada 2001), see Table 5. Concentrations of compound classes vary widely from less than ten ppm (amines) to tens of ppm (amino acids) to hundreds of ppm (carboxylic acids) (Cronin et al., 1988). Chromatographic analyses of virtually all classes of acyclic compounds reveal complex molecular assemblages containing homologous series of compounds up to $\mathrm{C}_{12}$ in some cases (carboxylic acids). 
Distinctive patterns of structural variation with molecular weight have emerged from several studies of these mixtures, none of which are exhibited by the classes of compounds used in living systems. Of these classes of compounds, amino acids have been used to illustrate these patterns, since any identifiable extraterrestrial biochemistry is likely to evolve catalytic polymers consisting of similar multifunctional building blocks. Studies of carbonaceous chondrites like Murchison have revealed some important chemical trends:

First, the abundances of compounds decrease with increasing carbon number (e.g. $\alpha-$ methyl branched and straight chain series of $\alpha$-amino acids (Cronin et al., 1988);

Second, the abundances of branched chain isomers far exceed those of the straight chain isomers (e.g. $\alpha$-methyl and $\alpha$-methyl branched isomers vs. straight chain $\alpha$ isomer);

Third, structural diversity dominates at the lower carbon numbers (e.g. acyclic monoamino acids).

Overall, 57 amino acids have been identified among the 159 possible $C_{2}$ to $C_{7}$ isomers (Cronin \& Chang 1993). Analytical sensitivity and diminishing abundances at higher molecular weight compounds limit further identifications. In contrast, life on Earth employs only 20 protein $\alpha$-amino acids, all of which have an $\alpha-H$ group. Biochemical counterparts account for less than a third of the total number identified. In addition, life on Earth uses a ubiquitous but restricted number of isomers from a few classes of organic compounds to fulfill its requirements for structure and function. Biosynthetic pathways manifest themselves in patterns of structural variation in these classes, just as abiotic pathways are reflected in the patterns exhibited by meteoritic compounds.

\section{Table 5 (put here)}

Structural diversity also occurs among cyclic meteoritic compounds like polycyclic aromatic hydrocarbons (PAHs), along with a variety of $\mathrm{C}_{15}$ to $\mathrm{C}_{30}$ branched alkylsubstituted mono-, di- and tri-cyclic alkanes (Cronin \& Chang, 1993). Polycyclic compounds together with the alkyl phosphonic and sulfonic acids comprise the most thermally stable species in the Murchison inventory. These compounds along with the IOM are the likeliest meteoritic compounds to survive delivery and subsequent geological processing on planets like the early Earth or Mars. Cooper et al. (2001) reported recently a variety of sugar polyols that are present in the CM meteorites Murchison and Murray in amounts comparable to amino acids.

Becker et al. (2000) have identified an extensive suite of high molecular weight carbon molecules $\left(\mathrm{C}_{60}\right.$ to $\mathrm{C}_{400}$ ) or fullerene in the Murchison and Allende carbonaceous chondrites. These caged carbon molecules contain trapped noble gases that are "planetary' in composition. 
A search for fullerene in sedimentary deposits associated with severe mass extinctions on the Earth (e.g. the Cretaceous/Tertiary, Permian/Triassic) has also resulted in the isolation and identification of fullerene with this unique planetary signature (Becker et al., 2001). Based on these findings, fullerenes appear to form in an extraterrestrial environment, are exogenously delivered to the Earth in some meteorites or comets and contribute to the inventory of volatiles identified in terrestrial planetary atmospheres. Thus, the notion that large impact events lead to complete devolatilization and destruction of most of their organic inventory. needs to be re-examined.

The observed patterns of variation in molecular structure and abundance with increasing carbon number in carbonaceous chondrites suggest synthesis routes involving small free radical initiators and intermediates (Cronin \& Chang, 1993; Curl, 1993). Such pathways tend to produce all possible structural isomers at lower carbon numbers by more or less random synthesis. Primary reactions in interstellar clouds could have produced mixtures of nitriles and other compounds. When exposed to liquid water on the meteorite parent body, the nitriles would have been converted to various substituted and unsubstituted carboxylic acids, including the amino acids. Other classes of compounds were likely to have formed in secondary reactions. For example, the Strecker synthesis of cyanohydrins and aminonitriles from $\mathrm{HCN}$, aldehydes/ketones and ammonia/amines, followed by hydrolysis, could account for the synthesis of some of the $\alpha$-amino- and $\alpha$-hydroxy acids. Amino acids substituted at more distant positions from the carboxyl group require other synthetic pathways.

The Tagish Lake meteorite, a very recent fall, showed a suite of soluble organic compounds including mono- and dicarboxylic acids, dicarboximines, pyridine carboxylic acids, sulphonic acid and aliphatic and aromatic hydrocarbons. There are practically no amino acids found in Tagish Lake and the meteorite shows evidence of substantial aqueous alteration yet no Strecker-type products. Its general composition and abundances differ strongly from other meteorites (Pizzarello et al. 2001, Ehrenfreund et al. 2001a).

Finally, we want to mention Interplanetary Dust Particles (IDP's) (Sandford \& Walker 1985, MacKinnon \& Rietmeijer 1987, Keller et al. 2000, Messenger 2000). These solid particles are considered to be as small fragments of objects stored at very low temperature for $4.56 \mathrm{Gyr}$. Among these objects are carbon-rich icy asteroids located at 3.5 - 5.2 AU, comet nuclei (formed at 20-30 AU) that enter the innermost regions of the solar system from the Oort cloud, and comets from the Kuiper belt (40$400 \mathrm{AU}$ ). IDP's could preserve unchanged the nature of the materials present in these bodies. Then, they are important indicators of silicate and organic materials present in the early phases of formation and evolution of our solar system. IDP's are composed of mixtures of many minerals, such as anhydrous pyroxene and olivine and hydrated layer silicates or by mixtures of sulfide, carbonate, sulfates as well as organics. 
Preliminary studies have suggested that grain surfaces could have an important role in chemical processes related to the prebiotic formation of molecules relevant to the origin of life on Earth. In particular, adsorption and catalysis on mineral surfaces have been shown to facilitate chemical reactions such as the polymerization of amino acids and nucleotides or the synthesis of complex molecules. IDP's are very porous, showing a high surface/volume ratio. Unlike large objects with the same porosity, IDP's survive during the entry in the Earth atmosphere. The presence in such grains of inorganic components with layer or porous cavity structure could provide large surface areas which represents a favorable local microenvironment where a high content of energy can be stored and utilized for prebiotic chemical reactions. 


\section{Delivery to Earth and prebiotic consequences}

Having inventoried the classes of identified organic molecules and mechanisms for their production in space, we turn now to the question of delivery of these materials to the terrestrial planets. Of particular interest are those mechanisms that may have been in play early in the Earth's history, when infalling organic compounds could have contributed to the chemical evolution leading to the origin of life.

Extraterrestrial organic material reaches the Earth in one of three ways: (1) As a component of dust grains and other particulates $(<1 \mathrm{~mm})$ that rain perpetually down to the planet and is ultimately of asteroidal and cometary origin, (2) as part of a cometary payload, and (3) incorporated in asteroid fragments (meteorites) that hit the planet. The proportion of material that survives these delivery processes without modification is unknown. The fraction that is altered on its way to the Earth's surface to form new compounds (e.g., McKay \& Borucki, 1997; Sugita \&. Schultz, $2001)$ is usually counted in the terrestrial prebiotic inventory. The notion of extraterrestrial delivery of organic matter to the early Earth has gained wide recognition because of the indisputable evidence that biomolecules, such as amino acids have reached the Earth's surface associated with meteorites (e.g. Botta et al. 2001). The overall contribution of these larger objects to the early Earth's inventory of organic matter has generally been considered to be insubstantial, however, because of the significant heating associated with passing through the Earth's atmosphere and the impact itself.

\subsection{Numerical simulations of impact events}

The principal argument against direct delivery of organic material to the Earth focuses on the very high temperatures expected in a high-velocity impact. Any impactor must have a minimum relative velocity of $11.2 \mathrm{~km} / \mathrm{s}$ (the escape velocity for the Earth), and this value constrains the possible temperature ranges a comet might experience. Most of the hydrodynamical models for impacts have simplified the nature of the impacting comet by treating it as a sphere of water ice. Because the pressures and temperatures generated in impact scale proportionally with the density of both the projectile and the impacted material, it follows that the conditions generated in a cometary impact will be less extreme than those generated by an asteroid impacting the Earth at a given speed and trajectory.

Chyba et al. (1990) calculated that maximum peak temperatures of $40,000 \mathrm{~K}$ would be reached if all the kinetic energy of an $18 \mathrm{~km} / \mathrm{s}$ comet were converted to heat. They suggested that this temperature multiplied by Boltzmann's constant is much higher than the activation energies typical for pyrolysis reactions of organic compounds (i.e., $k_{B} T \gg E_{a}$ ) and that an impact should induce complete breakdown of any organic molecules present. Subsequent modeling (Blank \& Miller, 1998; Pierazzo \& Chyba, 1999) suggests that special conditions such as oblique impacts would generate lower peak temperatures. 
When temperature alone is taken as a measure of chemical stability, the possible impact delivery flux would appear insignificant in comparison to terrestrial production mechanisms. Blank \& Miller (1998) reevaluated this kinetic argument using canonical activation volumes for bond breaking which, when positive, favor slower reaction kinetics associated with bond cleavage (e.g., pyrolysis) as pressure increases. On the basis of simple Arrhenius-like behavior, the rate of pyrolysis at $7,000 \mathrm{~K}$ and $100 \mathrm{GPa}$, conditions corresponding to planar impact of a water ice comet against a basaltic crust at escape velocity, should be comparable to that at $900 \mathrm{~K}$ and $1 \mathrm{~atm}$. Given the short time scale of an impact event, on the order of a second, organic compounds present in comets might survive brief exposure to high temperature and simultaneous high pressure, and their flux to the surface of the planet might therefore be significant. These authors considered the joint effect of pressure and temperature together and cautioned that intuition based on observations of material behavior at ambient pressure and high temperatures are likely to mislead.

A thermodynamic argument can also be made for the stability of organic compounds in impacts. If the Clapeyron slope for pyrolysis reactions is steeper than the slope of loci of states (Hugoniot curve) achieved by a shocked material, then the thermodynamic trajectory of the shock Hugoniot will favor the stability of reactants versus that of pyrolysis products, in accordance with Le Chatelier's principle. This argument also favors the combination of organic monomers to form polymers, including the combination of amino acids to form polypeptides.

Even at escape velocity the temperatures incurred in oblique collisions can be slight. Shock temperatures scale with the normal component of the impact velocity, and this can be made arbitrarily small as the impact vector approaches the horizontal (Blank $\&$ Miller, 1998). In this case, however, the kinetic energy of the tangential velocity component must be dissipated as heat. If that heat were distributed uniformly in the debris from the impactor, then temperatures circa $40,000 \mathrm{~K}$ might still occur. However, hydrodynamic phenomena (e.g., jetting; Miller, 1998) may distribute this energy heterogeneously. In fact, for some combinations of impact velocity and impact angle, parts of the outflow jet may experience very low peak temperatures (owing to the obliquity of the impact) and very low residual horizontal velocity (owing to the speed and orientation of the outflow jets). Blank \& Miller (1998) examined impact jets formed from a comet-earth collision and considered the possibility of soft-landing part of a comet (an idea proposed by Clark, 1988) which would become a localized reservoir of exogenous organic matter at elevated concentration levels in aqueous solution. Although the fraction of a given object that can be soft landed may be small (because the outflow jets represent a small fraction of the total impactor mass), it may be a small fraction of an arbitrarily large impactor. The probability of delivering a significant amount of material by the oblique impact mechanism 'described above is certainly small, particularly considering that the mean encounter velocity of short period comets with the Earth is not zero (as supposed by assuming the escape velocity), but distributed about a median value of $\approx 29 \mathrm{~km} / \mathrm{s}$ (Chyba et al., 1990; see also Steel, 1992). 
Most numerical models of large-scale impacts applied to the question of extraterrestrial delivery of organic material have invoked a comet collision with an ocean. While this impact scenario will allow a minimum of heating associated with impact, cometary organic material delivered in this fashion would merely add to the repository of organic material in the ocean rather than create an aqueous environment rich in organics. Blank \& Miller (1998) focused on the alternative impact scenario of a comet colliding at a low-angle with a rocky Earth surface in order to identify those regions of impactor most likely to bring a source of concentrated organic material to the surface of the planet. They noted that material jetted in the direction opposite to the impact would have a) the lowest temperatures and b) the lowest kinetic energy (and hence velocity) among the impacted cometary materials. The low temperatures would increase the likelihood for delivery of cometary water-ice in the form of liquid water. The low relative velocities would maximize possibilities for the delivery of material, in the form of water and associated organic compounds, without its being dispersed and diluted. In other words, the reverse-directed portion of the impacted comet offers the greatest potential for the extraterrestrial delivery of a concentrated aqueous pool rich in organic matter. If such a low-angle impacting comet were to deliver even a few percent of its material in this manner, the contribution to the overall organic flux would be significant, if not overwhelming (see Table 6).

\subsection{Laboratory Experiments}

Hypervelocity shock experiments have been conducted to characterize the survivability of organic elements in carbonaceous chondrites and water or water ice (to represent comets). These experiments typically involve a stationary sample in a metal container that is impacted by a high-speed metal bullet accelerated down a flight tube, or gun barrel, to velocities of several $\mathrm{km} / \mathrm{s}$. Pressures, generated by the metal-metal collision, are higher than would be produced by a direct impact of the sample materials alone; sample impact pressures are therefore higher for a given impact velocity to the reverberation, or "ringing up", of shock waves through the sample material as they reflect off the metal surfaces of the container. In general, the pressures generated in these experiments correspond to low-angle impact, while the temperatures would be very slightly lower, and the duration of the impact substantially shorter because it scales with the thickness of the impactor, which in the laboratory is on the order of millimeters.

There have been few impact studies on the shock chemistry of the organic materials associated with carbonaceous meteorites. Tingle et al. (1992) reported results from three shock experiments on cored pieces of the Murchison meteorite encapsulated in stainless steel capsules. The samples experienced peak pressures as high as 19, 20, and $36 \mathrm{GPa}$, respectively, through a sequence of shock reverberations (e.g., Gibbons $\&$ Ahrens, 1971), and recovered samples were analyzed by thermal desorption photo-ionization mass spectrometry. 
While there was negligible change in the organic constituents of samples shocked to peak pressures less than $20 \mathrm{GPa}$, approximately $70 \%$ of the original organic matter was lost from the sample exposed to a $36 \mathrm{GPa}$ impact, and the residual organic compounds appeared to have a lower $\mathrm{C} / \mathrm{H}$ ratio than the starting material, suggesting that the bulk composition had changed. As neither peak temperatures nor timetemperature-pressure histories of the samples are reported, the connection to chemical thermodynamics and kinetics cannot be made. Nonetheless, it is significant that organic material survived the extreme conditions of an impact to some degree. Peterson and others (1997) conducted a series of similar shock experiments over a pressure range of 3.5-32 GPa using powdered Murchison and Allende meteorite samples stripped of their original organic carbon and subsequently doped with wellcharacterized amino acids whose breakdown and reaction products were readily measured and distinguished. In these experiments, the abundance of residual amino acids was found to diminish substantially with high pressures, with typically $50 \%$ loss by $30 \mathrm{GPa}$. The formation of new daughter amino acids was inferred, in particular the decarboxylation of aspartic acid to form $\beta$-alanine.

Cooper et al. (2000) considered the survivability of four classes of organic compounds known to be indigenous to carbonaceous meteorites, by embedding them in a matrix of Murchison meteorite and subjecting them to laboratory shock pressures of up to $40 \mathrm{Gpa}$. They observed generally that survival of the compounds studied was inversely proportional to shock pressure (organic sulfur survived best, followed by organic phosphorus, polyaromatic hydrocarbons (PAHs) and amino acids).

Blank et al. (2001) simulated cometary impacts using water and/or ice doped with five amino acids (LD-2-Aminobutyric acid (AMB) and L-Norvaline (NOR), L-Lysine (LYS), L-Phenylalanine (PHE), and D-Proline (PRO)) and subjecting their aqueous samples to impact conditions ranging from 5-21 GPa and 412-870 $\mathrm{K}$ lasting several microseconds. Post-experiment analysis demonstrated survival of $>40-75 \%$ of the original amino acids; the cyclic amino acid, Phe, was the most reactive while the aliphatic amino acids, Lys and Nor, were the least. They also observed the persistence of the original population of amino acids to a greater extent in those capsule shocked to a higher peak pressure and temperature, contrary to the trend associated with the solid or powder samples mentioned above. Of possibly greater significance is the fact that the dominant reaction products identified by Blank and co-workers consisted of dipeptides formed from all possible combinations of the initial amino acids, with lesser amounts of higher-order peptides also present. A peptide bond forms via a dehydration synthesis reaction between the carboxy group of the first amino acid with the amino group of the second amino acid. Cyclic dipeptides (dikeopiperazines), formed by two amino acids linked together following loss of two water molecules, were also abundant among reaction products. These experiments demonstrated a mechanism by which impacts might actually increase the complexity of biologically relevant molecules from an initial starting pool. 


\subsection{Relevance to the prebiotic inventory of the Earth}

Dynamical considerations and experimental results thus support the possibility of organic carbon delivery in large impacts. The flux of organic matter to the Earth via comets and asteroids, averaged over the period of heavy bombardment prior to 3.8 Gyr, may have been as high as $10^{13} \mathrm{~kg} / \mathrm{yr}$ (calculated from data in Chyba et al., 1990; see also Zahnle \& Sleep, 1997). This estimate is substantially larger than the value of $\left(5 \times 10^{8}\right)$ to $\left(5 \times 10^{12}\right) \mathrm{kg} / \mathrm{yr}$ estimated to originate from the terrestrial environment, assuming the atmosphere was moderately oxidizing (Chyba \& Sagan, 1997). It also overshadows the possible contribution via IDPs, which until recently was perceived as the most efficient mechanism for extraterrestrial delivery (Anders, 1989) (see Table 6). Thus even a small fraction of organic material, delivered intact in a cometary body, could have a significant influence on the global budget. In addition, impact delivery may potentially localize this flux in space and time, leading naturally to concentrations of organic material significantly above levels easily achieved by endogenous production mechanisms.

\section{Table 6 (put here)}

Impact events may have played a significant role in the origins of life on the early Earth. Sufficiently large and fast impactors can erode planetary atmospheres (Melosh \& Vickery, 1989, Chyba \& Sagan, 1992). While this effect may have been devastating to life on Mars, it probably would not have prevented life from occurring many times on the Earth. In fact, impacts may have facilitated life by delivering 'intact' or synthesized organic compounds to the surface of the planet. However, geological approaches are limited in their ability to probe this period of interest because of the fragility of organic compounds and the paucity of the ancient sedimentary record. While a better preserved record of early planetary history may exist on Mars, the controversy raised from the recent appraisal of the ALH84001 Martian meteorite (McKay et al., 1996) illustrates that the question of early organic environments and life on Mars will likely be unanswered until samples can be returned to Earth.

Comets are the most promising source of exogenous compounds (cf. Thomas et al. 1997). Assuming that cometary nuclei have a $1 \mathrm{gm} \mathrm{cm}^{-3}$ density, a $1 \mathrm{~km}$-diameter comet would contain $2 \times 10^{11} \mathrm{M} H C N$, or 40 nmoles $\mathrm{cm}^{-2}$ of the Earth's surface. This is comparable to the yearly production of $\mathrm{HCN}$ in a reducing atmosphere from electric discharges, and would be quite important if the Earth did not have a reducing atmosphere. This calculation assumes a complete survival of the HCN on impact (Miller \& Lazcano, 2002). In fact, there is little understanding of what happens during the impact of such an object, but much of it would be heated to temperatures above $300{ }^{\circ} \mathrm{C}$ and thus would decompose $\mathrm{HCN}$ and other cometary compounds. However, these highly reactive chemical species could then be used as precursors in the abiotic syntheses of biochemical monomers. The extraterrestrial delivery of $\mathrm{H}_{2} \mathrm{O}$ via comets is discussed in section 10 . 
Studies of carbonaceous chondrites like Murchison demonstrate that some fraction of impacting bodies in the early solar system would have delivered organic compounds and volatiles to the surfaces of planets. The importance of the role of liquid water to the emergence of life on Earth, and perhaps Mars, lends special significance to the recent reports of the earliest known occurrence of liquid water (Mojzsis et al., 2001) and perhaps organics in the solar system. 'Prebiotic' evolution appears to have occurred early on some parent bodies of carbonaceous meteorites, and yet despite the abundance and diversity of organic compounds identified, the chemistry falls short of creating life. Nevertheless, samples from these objects offer a rich and accessible source of information for identifying model characteristics for organic matter and their environments during prebiotic evolution of planets like early Mars or even the moons of Jupiter and Saturn (e.g. Europa or Titan). 


\section{The hydrogen isotopic composition $(\mathrm{D} / \mathrm{H})$ in solar system organic matter and water}

The hydrogen isotopic composition (referred to as the deuterium-to-hydrogen ratio and noted $\mathrm{D} / \mathrm{H})$ of water and organic matter $(\mathrm{OM})$ is in principle an efficient tool to reconstruct the chemical synthesis of $\mathrm{H}$-bearing volatile components in the early solar system. From an "astrobiological" perspective, it is interesting to note that water and organic molecules are always associated in primitive planetary bodies like meteorites, comets and Interplanetary Dust Particles (IDP's). Accordingly if water and $\mathrm{OM}$, the essential components of life, originated in space, they originated in similar conditions.

This point of view is reinforced by the fact that, throughout the solar system, water and $\mathrm{OM}$ are systematically enriched in deuterium $\left(125 \times 10^{-6}<\mathrm{D} / \mathrm{H}<2000 \times 10^{-6}\right)$ relative to the "protosolar" $\mathrm{D} / \mathrm{H}$ ratio, i.e. to $\mathrm{D} / \mathrm{H}=25 \pm 5 \times 10^{-6}$ (Geiss \& Gloecker, 1998). In general, the more primitive the body is, the greater is the enrichments, except for the giant planets. No stellar (nuclear) process can yield such an isotopic enrichment, which is thus ascribed to chemical isotopic exchange reactions (on planets from which hydrogen can escape, enrichment can also occur as $\mathrm{H}$ escape more readily than $\mathrm{D}$ ). During these reactions, the deuterium of the protosolar hydrogen reservoir, which is in the form of the neutral atomic D or of molecular hydrogen $\mathrm{HD}$, is transferred to water and to OM. It is now generally accepted that such a large enrichment in deuterium can only be achieved at low temperature ( $\mathrm{T}<$ $200 \mathrm{~K}$ ), in media dominated by hot atom (ions or radicals) chemistry. In that respect, the deuterium enrichment demonstrate that solar system $O M$ and water share a common origin. However, this origin is not clearly understood.

In recent reviews (Robert et al., 2000; Robert, 2001), the D/H ratios in the insoluble organic matter (IOM) and in the water-bearing minerals (clays) embedded in the carbonaceous meteorites have been compared to their interstellar and cometary counterparts (only available for the volatile components). This isotopic distribution will not be discussed again in the present paper. Instead, we have selected the few number of solar system objects for which water and $\mathrm{OM} \mathrm{D} / \mathrm{H}$ ratios were both measured. Averaged published data along with their proper references on water and OM D/H ratios are presented in Table 7 and are discussed briefly in the notes of the Table. In Table 7, D/H ratios are all reported in units of $10^{-6}$ in order to be easily comparable. A discussion of these data can be found in Robert (2002). The comparison of water and $\mathrm{OM} \mathrm{D/H}$ ratios puts into light new isotopic relations from which a qualitative model accounting for the solar system $\mathrm{D} / \mathrm{H}$ variations can be developed.

In Figure 9, the data of Table 7 are reported with the water $\mathrm{D} / \mathrm{H}$ ratio as the $\mathrm{X}$-axis and the $\mathrm{OM} \mathrm{D} / \mathrm{H}$ ratio as the $\mathrm{Y}$-axis. The distribution of the data in Figure 9 cannot be accounted for by the classical $\alpha$-disk model of the protosolar nebula (Cassen, 1994; Dubrulle, 1992). In such models, the evolution of the water $\mathrm{D} / \mathrm{H}$ ratio in the protosolar gas has been modeled through the following isotopic exchange reaction (Lécluse \& Robert 1994; Drouart et al., 1999): 


$$
\mathrm{HD}+\mathrm{H}_{2} \mathrm{O} \leftrightarrow \mathrm{HDO}+\mathrm{H}_{2}
$$

This isotopic exchange is supposed to take place in the gas phase, i.e. before the condensation of water. It has been shown numerically that, within 1 million years, in a hot, dense and turbulent nebula where neutral-neutral reactions dominate the chemistry, the coupled action of isotopic exchange and isotopic homogenization (through the turbulent diffusion of water in the gas) can reproduce the variations of the water $\mathrm{D} / \mathrm{H}$ ratio, observed between comets and carbonceous meteorites (Drouart et al., 1999).

Using the same model, the evolution of the $\mathrm{OM} \mathrm{D} / \mathrm{H}$ ratio in the protosolar nebula was also numerically simulated in order to account for the cometary value. Taking appropriate initial interstellar $\mathrm{D} / \mathrm{H}$ ratios for water and $\mathrm{OM}(\mathrm{HCN}$ in the case of comets), the model yields the observed cometary $\mathrm{D} / \mathrm{H}$ ratios. It is important to note that, in this model, the initial isotopic conditions are defined by two fixed values for water and $\mathrm{OM} \mathrm{D} / \mathrm{H}$ ratios. These ratios are interpreted as the interstellar $\mathrm{D} / \mathrm{H}$ ratios, carried by water and $\mathrm{OM}$, that predates the formation of the solar system. During the evolution of the nebula, no fresh interstellar water or OM is supposed to be injected in the gas. In this respect, the model represents an evolution in a closed system. For given parameters describing the initial physical conditions of the nebula (its radius, mass, accretion rate and coefficient of turbulent viscosity), there is only one single pathway describing the relations between water and $\mathrm{OM} \mathrm{D} / \mathrm{H}$ ratios. An example of such pathways is shown in the Figure 9 as a dashed line (after Mousis et al., 2000). According to this model, the $\mathrm{D} / \mathrm{H}$ ratios of grains move along this dashed line as a function of time and of heliocentric distance at which $O M$ and water condensed on these grains.

A systematic study of this type of calculated pathways (F. Hersant pers. comm.), have shown that $\alpha$-disk models cannot account for the distribution of the D/H ratios reported in Figure 9 because (1) two given initial water and $\mathrm{OM} \mathrm{D} / \mathrm{H}$ ratios correspond to a unique evolution pathways (as shown by the dashed line) (2) whatever the initial conditions, no isotopic variation in the water $\mathrm{D} / \mathrm{H}$ ratio is expected between 0.5 and 4.0 A.U. The case of the Renazzo meteorite (noted CR in Figure 9) is in clear contradiction with this theoretical prediction whose water D/H ratio is twice the values of other carbonaceous meteorites. It seems therefore unavoidable that, beside the isotopic exchange within the protosolar molecular hydrogen, an additional process has played a role in establishing the D/H ratio of solar system objects. Such a possible process is now presented.

First, it must be noted that, according to the Figure 9, water and OM are not related by a unique isotopic fractionation process which should give a linear (proportional) correlation in this diagram with the isotopic fractionation factor being the slope of the correlation. In Figure 9, two solid lines were arbitrarily drawn. They do not represent possible correlations in the diagram but define the upper and lower limit of the isotopic variations: the upper limit is defined by the comets and by one IDP and the lower limit by the carbonaceous meteorites. They also correspond to the inner and outer regions of the solar system (meteorites and comets respectively; the origin of IDP's are still in debate). 
Let us assume that these lines result from two coupled isotopic fractionation : (1) a fractionation between $\mathrm{OM}$ and $\mathrm{H}_{2}$ and (2) another one between water and $\mathrm{H}_{2}$. That is :

$\alpha_{\text {water-H2 }}=(\mathrm{D} / \mathrm{H})_{\mathrm{water}} /(\mathrm{D} / \mathrm{H})_{\mathrm{H} 2}$

$\alpha_{\mathrm{OM}-\mathrm{H} 2}=(\mathrm{D} / \mathrm{H})_{\mathrm{OM}} /(\mathrm{D} / \mathrm{H})_{\mathrm{H} 2}$

Coupling these two isotopic exchange processes yields:

$\alpha_{\mathrm{OM}-\mathrm{water}}=(\mathrm{D} / \mathrm{H})_{\mathrm{OM}} /(\mathrm{D} / \mathrm{H})_{\text {water }}$

The slopes of the two lines drawn in Figure 9 may thus stand for two different values of $\alpha_{\mathrm{OM}-w a t e r}$. Since the isotopic fractionation factor $\alpha$ depends exclusively on the temperature, the two lines can be viewed as isotopic thermometers.

At a first order of approximation, let us express the isotopic fractionation factors as :

$\alpha_{\text {water-H2 }}=(\mathrm{D} / \mathrm{H})_{\text {water }} /(\mathrm{D} / \mathrm{H})_{\mathrm{H} 2}=\operatorname{Exp}\left(\Delta \mathrm{E}_{\mathrm{Water}} / \mathrm{T}\right)$

$\alpha_{\mathrm{OM}-\mathrm{H} 2}=(\mathrm{D} / \mathrm{H})_{\mathrm{OM}} /(\mathrm{D} / \mathrm{H})_{\mathrm{H} 2}=\mathrm{Exp}\left(\Delta \mathrm{E}_{\mathrm{OM}} / \mathrm{T}\right)$

Let us assume that, at a given temperature, the $\mathrm{D} / \mathrm{H}$ of $\mathrm{OM}$ and water move linearly in Figure 9. That is to assume: at a given temperature $\mathrm{T}$, the position on the line is kineticaly controlled by the rates of the isotope exchange. The slope $s$ of the line is:

$s=\operatorname{Exp}\left[\left(\Delta E_{O M}-\Delta E_{W a t e r}\right) / T\right]$

It is possible to calculate a value for $\Delta \mathrm{E}_{\mathrm{OM}}$ and $\Delta \mathrm{E}_{\text {Water }}$ that will fulfill two conditions: (1) matching the slopes of the two solid lines reported in Figure 9 (2) giving equilibrium values for $\alpha$ 's higher than the highest $\mathrm{D} / \mathrm{H}$ ratios on each lines i.e. higher than $\mathrm{CR}$ and Cometary $\mathrm{D} / \mathrm{H}$ ratios. An example of a possible solution is $\Delta \mathrm{E}_{\mathrm{OM}}=370 \mathrm{~K}$ and $\Delta \mathrm{E}_{\mathrm{Water}}=280 \mathrm{~K}$, giving temperatures of $120 \mathrm{~K}$ and $35 \mathrm{~K}$ for the two lines. These temperatures must be considered as upper limits since for $\Delta \mathrm{EW}_{\mathrm{W}}$ $>280 \mathrm{~K}$, temperatures $<120 \mathrm{~K}$ and $<35 \mathrm{~K}$ would correspond to the two lines drawn in Figure 9 (with $\Delta \mathrm{E}^{\prime}$ 's still fulfilling the two conditions). Interestingly, the $\triangle \mathrm{E}_{\mathrm{OM}}$ and $\Delta \mathrm{EW}_{\mathrm{W}}$ ater are close to the following known isotopic exchange ion-molecule reactions:

$$
\begin{array}{lll}
\mathrm{H}_{3}{ }^{+}+\mathrm{HD} & \rightarrow \mathrm{H}_{2} \mathrm{D}^{+}+\mathrm{H}_{2} & \left(\Delta \mathrm{E}_{\text {Water }}=227 \mathrm{~K}\right) \\
\mathrm{CH}_{3}{ }^{+}+\mathrm{HD} \rightarrow \mathrm{CH}_{2} \mathrm{D}^{+}+\mathrm{H}_{2} & \left(\Delta \mathrm{E}_{\mathrm{OM}}=370 \mathrm{~K}\right)
\end{array}
$$


Such ion-molecule reactions that characterize the interstellar chemistry between 10 and $100 \mathrm{~K}$, are known to be a fast event at these low $\mathrm{T}$ (Brown and Rice, 1981; Guélin et al., 1982; Rodgers \& Millar, 1996; Willacy \& Millar, 1998). Based on these considerations, a scenario for the isotopic fractionation of hydrogen in the early solar system can be proposed. These calculated isotopic temperatures stand for the last isotopic exchange with the solar system hydrogen reservoir. Thus, these temperatures do not rule out the fact that water (and OM) was originally formed at $10-20 \mathrm{~K}$ in the ISM and was then isotopically re-equilibrated with $\mathrm{H}$ during the collapse of the interstellar cloud that yielded the formation of the protosolar nebula. Such re-equilibration is indeed observed in water in hot cores regions (cf. Table 7).

According to the present interpretation, inner solar system water and OM were isotopically fractionated to a different extent but both at the same temperature (120 $\mathrm{K})$. In the outer solar system, a similar type of isotopic fractionation occurred but at much lower temperature $(35 \mathrm{~K})$. The LL meteorites fall in between the two lines, attesting that their organic matter and water formed at temperatures intermediate between carbonaceous meteorites and comets. Since the isotopic temperatures for inner solar system objects (meteorites) are higher than for outer solar system objects, the deuterium enrichment seems to be governed by a heliocentric parameter. This observation can be understood in the light of modern theories of star formations.

Astronomical observations provide compelling evidence that all young stars (YSO; Young Stellar Objects) go through a T-Tauri phase and emit an intense flux of Xrays that "waters" the surface of their surrounding disk (André \& Montmerle, 1994). Calculations show that $\mathrm{X}$-rays penetrate deep into the gaseous disk due to Compton scattering and that the ionization rate of the disk greatly exceeds that of the ISM (Chiang et al., 2001; Glassgold et al., 2000). The degree of ionization, the thickness and the temperature of the ionized disk varies as a function of the distance to the sun. Under those conditions, ion-molecule reactions such as those schematically depicted by reactions (8) and (9) can rapidly proceed. Once formed at the surface of the disk, organic and water molecules can sink in the deeper, denser and neutral regions of the disk - the so-called protosolar nebula (Cassen, 1994; Dubrulle, 1992; Drouart et al., 1999) - where they are protected from solar ionizing particles. This interpretation may not be in conflict with classical alpha-disk models of the protosolar nebula such as the one developed by Drouart et al; (1999). However, it strongly suggests that the nebula cannot be treated as a closed system: chemical species, formed via nonthermal chemical reactions, were injected into the protosolar gas, along its temporal and spatial evolution. The preservation of isotopic thermometers in such an open system - as depicted in the Figure 9 - remains an open issue. Indeed, isotopic systems may have eventually lost the memory of their reaction temperatures since their $\mathrm{D} / \mathrm{H}$ ratios can be partially reset via reaction (1). Such an issue is beyond anything, which can be calculated with the presently available models of the protosolar nebula. Nevertheless, the proposed solar irradiation scenario offers a plausible interpretation pointing toward a local and a common origin for both water and organic matter. 


\section{A habitable planet and the conditions for life}

In the previous sections we have compiled information on astrophysical conditions and the chemical processes that allow the formation, distribution and survival of prebiotic matter and described a scenario for extraterrestrial delivery to the early Earth. Intelligent life might be unique to our planet. But it may very well be that simple life forms have emerged elsewhere. Comparative planetology will certainly help us in the near future to understand more about the evolution of life and our existence.

What exactly do we mean by an Earth-like planet? The basic criteria are straightfoward. We want a rocky planet with open bodies of liquid water on its surface. To achieve this, we require a nearly circular orbit about a sun-like star, so the planet experiences a uniform temperature throughout its year. Obviously some deviations from this state are acceptable, but an eccentricity that would freeze the planet's water in one part of its orbit or boil it in another would not be. The Earth's distance from the sun is critical - Venus is $25 \%$ closer and this is too close for comfort - liquid water is not stable on the planet's surface. A runaway greenhouse develops that leads to boiling of the oceans and the loss of water through photodissociation in the planet's upper atmosphere, with consequent escape of hydrogen. A distance much beyond that of Mars would lead to permanent freezing of the oceans. However, the main problem for Mars is not its distance from the sun but its mass, which is too small to withstand the effects of early bombardment by planetesimals. These impacts can cause the wholesale erosion of the atmosphere, leaving Mars with the thin envelope of atmosphere we find today.

Mass and distance from the parent star are thus essential criteria, but are they sufficient? For life to begin and evolve, we need a supply of biogenic elements, specifically $\mathrm{C}, \mathrm{H}, \mathrm{N}, \mathrm{O}$ and various minor species such as $\mathrm{Ca}, \mathrm{P}, \mathrm{S}$, etc. The latter are provided by the rocks and may be assumed to be present on any rocky planet, although it is clearly advantageous (again!) to have a planet sufficiently massive to have undergone differentiation and produced a crust that concentrates some of these important elements. But it is the first four elements that merit major concern.

HOCN are the four most abundant, chemically active elements in the universe, see section 1. They are also the most abundant elements in all forms of life known to us. Yet, with the exception of $O$, these elements are rare on Earth. Relative to silicon, the common rock-forming element, hydrogen, nitrogen, carbon and oxygen are respectively depleted by factors of $10^{7}, 10^{5}, 10^{3}$ and 10 compared with abundances in the sun. Oxygen is the least depleted as it is an essential component of silicates, the most common type of rock on the planet.

The hydrogen and oxygen essential to life are found in their greatest abundance in $\mathrm{H}_{2} \mathrm{O}$, as life does not extract $\mathrm{O}$ from silicates. But what about $\mathrm{C}$ and $\mathrm{N}$ ? Life without $\mathrm{C}$ is unimaginable to us, yet the origin of this element on the Earth is somewhat mysterious. It is relatively rare in rocks, without biological assistance it occurs most abundantly as graphite. The great deposits of carbon on Earth are either carbonates consisting of $\mathrm{CO}_{2}$ that was once in the atmosphere - or biogenic compounds such as coal and petroleum. 
Nitrogen is even more absent from geology, with no major minerals and nitrate deposits that are overwhelmingly biogenic. So if they didn't come in with the rocks, how did $\mathrm{C}$ and $\mathrm{N}$ reach the planet?

Most of the $\mathrm{H}_{2} \mathrm{O}$ may well have been brought in the form of hydrous minerals or adsorbed water. We may be able to rule out comets as the major carrier by comparing $\mathrm{D} / \mathrm{H}$ in cometary water with $\mathrm{D} / \mathrm{H}=1.55 \times 10^{4}$ in the oceans. In the three Oort cloud comets measured thus far, $\mathrm{D} / \mathrm{H}=3.2 \pm 0.5 \times 10^{-4}$, essentially twice the value found in the oceans. On the other hand, near-surface water on Mars appears to have the same isotopic ratio as the comets, suggesting that both Mars and Earth received some cometary endowment. On Earth, the cometary water would have been thoroughly mixed with water from the planet's interior, thanks to the geological activity (eruption, subduction) on this planet. On smaller Mars, the hydrosphere has remained isolated from the lithosphere, as demonstrated by studies of the oxygen isotopes in Martian meteorites.

To determine how much cometary water reached the Earth, we need to know the value of $\mathrm{D} / \mathrm{H}$ in the water that was brought in by the rocks. In the extreme case that this water fully equilibrated with the nebular hydrogen, we would have $\mathrm{D} / \mathrm{H}=2.6 \times$ $10^{-5}$ and $50 \%$ of the oceans would have been supplied by comets. Another approach is to assume that comets brought in the Earth's nitrogen and carbon and ask how much water would accompany these elements. In the comets studied thus far C/O appears to have the solar value of 0.33 , while nitrogen is depleted. We can evaluate the total amount of carbon that has cycled through the Earth's atmosphere, the result is $\mathrm{C} \approx 10^{23} \mathrm{gm}$. This implies $4.5 \times 10^{23} \mathrm{gm}$ of $\mathrm{H}_{2} \mathrm{O}$, compared with the mass of ocean: $1.5 \times 10^{24} \mathrm{gm}$. This calculation gives an upper limit of $30 \%$ to the cometary contribution.

A closer specification will be possible when we know the abundances and isotopic architecture of the heavy noble gases krypton and xenon in comets. If it turns out that $\mathrm{Kr} / \mathrm{Xe}$ and the relative abundances of the Xe isotopes are the same in comets as they are in the atmospheres of Earth and Mars, there will then be a prima facie case that comets indeed impacted the Earth, bringing their volatiles with them. Furthermore, it will be possible to estimate the total $\mathrm{H}_{2} \mathrm{O}$ (or $\mathrm{C}$ or $\mathrm{N}$ ) brought by comets simply by ratioing to one of these heavy noble gases.

If comets indeed brought at least some fraction of the water on Earth and the other inner planets, these icy messengers must have delivered some carbon and nitrogen as well. As already mentioned $\mathrm{C} / \mathrm{O}$ should have the solar value in comets, while N/O may be depleted by about a factor 5 (Geiss 1989, Krankowsky 1991). These numbers are derived from investigations of Halley's comet by the Giotto spacecraft and by ground-based telescopes, so there is an assumption here that Halley is truly representative of all Oort cloud comets. 
In fact, at least 4 different types of comets are known to exist, based on relative abundances of the radicals observed in visible spectra (A'Hearn et al. 2001). These radicals are daughter products of molecules studied in the radio region or in situ. Thus, we should worry that more fundamental differences in composition may exist that we have not yet identified. This should not be surprising, in view of the immense span of more than $30 \mathrm{AU}$ over which icy planetesimals could have formed in the outer solar nebula, corresponding to a possible range in temperature from 30 to 150 $\mathrm{K}$. This temperature range permits major differences in amounts of trapped ambient gases in condensing ice, if comets formed from interstellar ice grains that evaporated as they entered the solar nebula and then subsequently recondensed (Lunine et al. 1991, Owen \& Bar-Nun 1995). Despite the uncertainties, the observations of comets recorded thus far indicate that all of them - with the possible exception of Tanaka $1983 \mathrm{a}$ - contain volatile nitrogen and carbon compounds in addition to water ice. 


\section{The origin of life}

\subsection{The antiquity of terrestrial life}

The basic constituents of life on planet Earth include the genetic material DNA and RNA as well as proteins. DNA is structured in a double helix formed from three different components, nucleobases, sugars and phosphates. Proteins represent 3dimensional networks of amino acids. Although amino acids are found in meteorites together with pre-sugars and traces of nucleobases, the upper limits for those compounds elsewhere in space indicate that their direct exogenous delivery might not play an important role for life on our planet. Additionally, very little is known about about phosphorus chemistry in space. The analysis of meteorites definitely proves that extraterrestrial matter has arrived intact on our planets and other solar system bodies. But it is far from understood if this material was in any way necessary for the origin of life.

Understanding the origin of life requires understanding the conditions under which it took place, but there is no geological evidence of the atmospheric composition, surface temperature, ocean $\mathrm{pH}$ values, and other general and local environmental conditions which may or may not have been important for the appearance of the first living systems on Earth. Nevertheless, the presence of cyanobacteria-like microfossils in the 3.5 billion years old Australian Apex sediments (Schopf 1993) demonstrates that the emergence and early diversification of life on our planet did not require more than a few hundred million years. Together with the inclusions enriched in light isotopic carbon in $3.86 \times 10^{9}$ billion years old samples from southwestern Greenland (Mojzsis et al. 1996), these results show that a widespread, complex, and highly diversified Archaean microbiota was thriving soon after the Earth had formed. Such rapid development speaks for the relatively short timescale required for the origin of life, and suggests that the environmental conditions under which it took place were shaped to a considerable extent by the astrophysical conditions of the inner solar system and the astrochemical evolution of the early Earth.

\subsection{The heterotrophic theory and the primitive soup}

The lack of direct information and the seemingly insurmountable obstacles surrounding the understanding of the origin of life have led to alternative and competing suggestions regarding how life emerged and what were the defining characteristics of the first organisms. Although discussions of panspermia, i.e., the transfer of organisms from one planet to another, are periodically resurrected, the leading hypotheses for the origin of life have been developed mainly within the historical mainstream of an evolutionary development. In spite of their diversity, proposals for a prebiotic soup, for the role of submarine hydrothermal vents, or for the ultimate extraterrestrial origin of organic compounds have as a common background assumption the idea that abiotic organic compounds were a necessary preamble for the appearance of life. 
This is the basic tenet of the heterotrophic description of the origin of life, first proposed by Oparin and Haldane in the 1920s', according to which the first lifeforms were not able to synthetize their own compounds, but were formed from pre-existing organic compounds of abiotic origin (Miller \& Lazcano, 2002).

\subsection{An autotrophic origin of life?}

Relatively few theories assuming an autotrophic origin of life, i.e. that the very first organisms were able to fix $\mathrm{CO}_{2}$ and synthesize their own organic compounds, have been proposed as alternatives to the idea of a heterotrophic viewpoint. The most articulate autotrophic hypothesis stems from the work of Günter Wächtershäuser, who has argued that life began with the appearance of an autocatalytic twodimensional chemolithotrophic metabolic system based on the formation of the highly insoluble mineral pyrite.

The reaction $\mathrm{FeS}+\mathrm{H}_{2} \mathrm{~S}=\mathrm{FeS}_{2}+\mathrm{H}_{2}$ is a very favourable one, with a highly exergonic character with a standard free energy change $\Delta G^{0}=-9.23 \mathrm{kcal} / \mathrm{mol}$, which corresponds to a reduction potential $\mathrm{E}^{\mathrm{o}}=-620 \mathrm{mV}$. Thus, the $\mathrm{FeS} / \mathrm{H}_{2} \mathrm{~S}$ combination is a strong reducing agent, and has been shown to provide not only an efficient source of electrons for the reduction of organic compounds under normal pressures and relatively low temperatures, but also to promote the formation of peptide-bonds that result from the activation of amino acids with carbon monoxide and $(\mathrm{Ni}, \mathrm{Fe}) \mathrm{S}$, as well as the fixation of carbon monoxide into activated acetic acid by a mixture of coprecipitated NiS/Fe/S (Huber \& Wächtershäuser 1998).

None of these experiments (which take place in an aqueous environment) proves by itself that both enzymes and nucleic acids are the evolutionary outcome of surfacebounded metabolism, as hypothesized by Wächtershäuser. These results are also compatible with a more general, modified model of the primitive soup in which pyrite formation is recognized as an important source of electrons for the reduction of organic compounds, i.e., the experimental results achieved so far using the $\mathrm{FeS} / \mathrm{H}_{2} \mathrm{~S}$ combination are consistent with a heterotrophic origin of life.

\subsection{Prebiotic chemistry}

Starting with Wohler's classical laboratory synthesis of urea in 1828 , the formation of organic compounds under conditions which could be accepted nowdays as primitive had been investigated during the 19th century. The list includes, among others, Strecker's formation of alanine from acetaldehyde, ammonia, and hydrogen cyanide, and Butlerov's laboratory synthesis of sugars using formaldehyde as starting material. However, it was not until 1953 when the first successful synthesis of organic compounds under plausible primordial conditions was accomplished by the action of electric discharges acting for a week on a mixture of $\mathrm{CH}_{4}, \mathrm{NH}_{3}, \mathrm{H}_{2}$, and $\mathrm{H}_{2} \mathrm{O}$; racemic mixtures of several proteinic amino acids were produced, as well as hydroxy acids, urea, and other organic molecules, see Figure 10 (Miller \& Urey 1953). 
Miller's 1953 experiment was followed a few years later by the demonstration of rapid adenine synthesis by the aqueous polymerization of $\mathrm{HCN}$ under basic conditions. The role of HCN in prebiotic chemistry has been further supported by the discovery that the hydrolytic products of its polymers include several amino acids, purines, and orotic acid, which is a biosynthetic precursor of the pyrimidine uracil, a constituent of RNA.

A potential prebiotic route for the synthesis of cytosine in high yields is provided by the reaction of cyanoacetylene with urea, specially when the concentration of the latter is increased by simulating an evaporating pond (Miller \& Lazcano, 2002).

The ease of formation of amino acids, purines, and pyrimidines involving a large number of steps from few precursors under simple experimental conditions strongly suggests these components were present in the prebiotic environment. As a number of simulation experiments suggest, additional products of prebiotic chemistry probably included urea, alcohols, carboxylic acids, sugars, a wide variety of aliphatic and aromatic hydrocarbons, and branched and straight fatty acids, including some which are membrane-forming compounds.

The list also includes several highly reactive derivatives of $\mathrm{HCN}$, such as cyanamide $\left(\mathrm{H}_{2} \mathrm{NCN}\right)$ and its dimer $\left(\mathrm{H}_{2} \mathrm{NC}(\mathrm{NH}) \mathrm{NH}-\mathrm{CN}\right)$, dicyanamide (NC-NH-CN), and cyanogen $(\mathrm{NC}-\mathrm{CN})$, which are known to catalyze polymerization reactions.

The significance of prebiotic simulation experiments is supported by the occurrence of the large array of protein and non-protein amino acids, carboxylic acids, purines, pyrimidines, hydrocarbons, and other molecules in the 4.6 billion years-old Murchison meteorite, a carbonaceous chondrite which also yields evidence of liquid water. These meteoritic compounds make it plausible, but do not prove, that a similar synthesis took place on the primitive Earth. Together with the ever increasing complexity of organics found in the ISM, we see that nature easily makes the first steps in prebiotic chemistry.

\subsection{Extraterrestrial sources of organic compounds}

The abiotic syntheses of amino acids, purines, pyrimidines, and other compounds are very efficient if strongly reducing $\left(\mathrm{CH}_{4}+\mathrm{N}_{2}, \mathrm{NH}_{3}+\mathrm{H}_{2} \mathrm{O}\right.$, or $\left.\mathrm{CO}_{2}+\mathrm{H}_{2}+\mathrm{N}_{2}\right)$ gaseous mixtures are used in simulation experiments. However, the possibility that the primitive atmosphere was non-reducing, as currently favoured by planetary scientists, does not create insurmountable problems, since the primitive soup could still form. For instance, geological sources of hydrogen, such as pyrite, may have been available; in the presence of ferrous iron, a sulfide ion ( $\mathrm{SH}^{-}$) would have been converted to a disulfide ion $\left(S_{2}\right)$, thereby releasing molecular hydrogen. It is also possible that the impacts of iron-rich asteroids enhanced the reducing conditions, and that cometary collisions created localized environments favouring organic synthesis. 
Of course, the question is not what was the source of organic compounds, but what percentage came from each source (see also section 8 and Table 6). For instance, although the total amount of formaldehyde, hydrogen cyanide, acetaldehyde, cyanoacetylene and other prebiotic compounds in a given interstellar cloud is high, any of these compounds surviving the formation of the solar system would only have reached the Earth as minor constituents of comets, carbonaceous meteorites or IDPs. The Murchison meteorite contains approximately $1.8 \%$ organic carbon, but most of this is a polymer. There are only about 100 parts per million of amino acids (which represents, assuming a void volume of $10 \%$ and a density of approximately 2.0 , a density of $0.10 \mathrm{gm}$ amino acids per $\mathrm{kg}$ of meteorite, or $2.0 \times 10^{-2} \mathrm{M}$ of amino acids) (Miller \& Lazcano, 2002).

Any origin of life theory based on the evolution of a self-replicanting genetic system involves the use of chemically stable compounds and stabilizing micro-environments. Hydrogen cyanide ( $\mathrm{HCN}$ ) chemistry provides a preferential route for the prebiotic synthesis of purines and pyrimidines, see above. Synthesis from formamide of purine and pyrimidine derivatives under catalytic conditions provided by the presence of dust grains could represent a more efficient prebiotic route than $\mathrm{HCN}$ chemistry. Saladino et al., (2001) proposed an experimental scenario that builds on hydrogen cyanide ( $\mathrm{HCN}$ ) chemistry. Formamide, easily formed by the hydrolysis of $\mathrm{HCN}$, can synthesize purine and pyrimidine derivatives in the presence of various metallic oxides at fairly low temperature $\left(110-160^{\circ} \mathrm{C}\right)$. In this study it was firstly demonstrated that cytosine can be formed from formamide. Owing to the relatively mild chemical conditions of the formation, this has important implications for the nitrogen base stabilities. Saladino et al., $(1996,2001)$ have shown that other purines and pyrimidine compounds do form through a novel set of chemical reactions. In particular, while calcium carbonate $\left(\mathrm{CaCO}_{3}\right)$ gave purine as the only recovered product, a different reaction pattern was observed with silica $\left(\mathrm{SiO}_{2}\right)$, where pyrimidine derivatives became the main reaction products. On the other hand, when the reactions were performed in the presence of kaolin and zeolite ( $Y$ type), purine was again obtained as the main product. Thus, a wide range of selectivities and yields could be obtained depending on the catalyst used. The key finding is that purines and pyrimidines are part of a cycle of reactions which, starting from formamide, may lead to their synthesis or (depending on the conditions) to their degradation, eventually yielding the starting compound formamide. 


\subsection{Chirality and the origin of extraterrestrial organics}

A fundamental characteristic of life is the homochirality of most of its building blocks. Various theories have been proposed to explain its origin (Bonner 1991); most of them require a chemical amplification scheme, but they differ in the origin of the initial excess to be amplified: random fluctuation, electroweak interaction effect, or extraterrestrial input. Enantiomeric excesses have up to now been found only in meteoritic matter (e.g. Engel \& Macko 1997, Cronin \& Pizzarello 1997 but are searched for in micrometeorites (eg Vandenabeele-Trambouze et al. 2001) and comets (COSAC experiments on-board ESA mission ROSETTA, Thiemann \& Meierhenrich 2001).

Early experimental approaches on the syntheses of organic compounds do not produce chiral products (Miller \& Urey, 1953). Recent evaluation of abiotic mechanisms proposed for the origin of chiral molecules on the primitive Earth concluded that such approaches are not likely to occur in nature (Bonner et al. 1999). The absence of chirality in products of prebiotic evolution experiments strengthened the presumption that natural abiotic synthesis invariably produces. racemic. compounds. Indeed, early analysis of Murchison amino acids declared them racemic (e.g., Kvenvolden et al., 1970).

In 1997, Cronin \& Pizzarello reported modest L-enantiomeric excesses of 2 to $9 \%$ in some amino acids in the Murchison meteorite. They avoided the problems of contamination by making measurements on 2-amino-2,3-dimethylpentanoic acid, $\alpha$ methyl norvaline and isovaline. All three of these compounds are $\alpha$-methyl substituted; the first two have no known biological counterparts and the third has a restricted distribution in fungal antibiotics. Bailey et al. (1998) has suggested that the observed enantiomeric excesses could have been induced by circularly polarized light arising from dust scattering in regions of high mass star formation. These sources occur more widely than do the supernova remnants or pulsars that were first proposed by Rubenstein et al. (1983) as sources of circularly polarized synchrotron radiation. However, both theories do not take into account that amino acids are very fragile compounds, which are easily destroyed by particle radiation and even by low energy UV photons (Ehrenfreund et al. 2001b). An extraterrestrial origin of chirality is strongly debated. The majority of the scientific community favours the idea that the left-handed configuration of several bio-compounds appeared as an accident during the assembly of prebiotic compounds on the early Earth. 


\subsection{Perspectives for prebiotic life studies from experiments and modelling in biotechnology and soft nanotechnology \\ 11.8}

The reconstruction of critical events in the origin of life is challenging, since the evidence is always indirect: there is no fossil record from prebiotic life. Alternatively, plausible scenarios can be inferred by deconstruction of existing living systems. In this top-down approach, advocates of 'World' theories extract a feature of life, thought to be central, and propose that the selected feature developed first and the rest followed. The 'RNA World' hypothesis assumes self-replication information storage was first (Gesteland et al, 1999), the 'Lipid World' confinement was first- (Segre et al, 2001), 'Protein World' - protein microspheres first- (Fox 1988), and the 'Thioester World' - metabolism first (de Duve, 1991). The World models profit enormously from the advances in genomics, proteomics and metabolomics. We have only seen the beginning of the understanding of the fundamental relations between the organisational units in a living cell, the distribution of properties over different species, and the rebuilding of ancient common life-determining factors. There can be no doubt that such 'bio-archeology' will lead to a critical evaluation and fine-tuning of the World models: perhaps one will survive - or perhaps all will die. The world models all rely heavily on some sort of primitive biological machinery, and it is therefore unclear whether the topdown approach will allow us to look into the primordial prebiotic conditions, before the onset of biology.

On the theoretical side, with the advance of cheap computer power and simulation methods since the early seventies, there has been an explosion of speculative computer simulation models and analytical models for complex collective behaviours of prebiotic chemical mixtures (Dyson 1985). The popular versions are always variations of Eigen's original Hypercyle concept (Eigen \& Schuster 1979). Here the general feature is a compound reaction-diffusion model, embodied in a series of coupled (partial) differential equations, for a reacting mixture of complex bio-organic molecules. The set of equations is integrated on a computer, given parameters such as reaction rates and diffusion coefficients. The complex mixture should have a sufficient diversity, so that certain molecular types catalyse the formation of others. In a proper region in parameter space, diffusion and mutual catalysis will lead to self-sustaining autocatalytic networks, and these networks may contain information, merge, split, reproduce etc: life will follow spontaneously. There is an interesting connection with novel approaches in metabolomics, proteomics, chemical engineering and random or combinatorial chemistry, which makes the theoretical approach not only relevant for origin of life studies, but also for the development of new technology. In genomics and bioinformatics, projects such as E-Cell (http://www.e-cell.org) endeavour to reproduce the features of a living organism in a computer model. The E-Cell models need careful calibration with experimental data, but once this is done, they allow for a virtual test of the chemical conditions necessary for life. 
Can the computer models also serve as rational design tools for the preparation of a self-sustaining chemical system $a b$ initio in the laboratory, without reference to, or using chemicals from, known biological origin? If yes, this would mean a major advance in our understanding of complex chemical networks, the origin of life on Earth, and the possibility for life elsewhere in the universe. The task is to invent a purely synthetic present-day system, capable of performing basic phenomena of selfsustaining chemical networks: increase complexity by a bottom-up approach, rather than decompose complexity as in the top-down bio-archeology.

A different perspective comes from advances in design of smart soft nano-materials. For example, when the interest is in preparing a drug delivery capsule, capable of self-propagation, recognition and response, there are obvious connections with designing life (Artificial Cell projects), and the same methods can be used in both fields with mutual benefit (Szostak et al., 2001). In Figure 11 is the simulation result of a drug delivery microscopic hydrogel, consisting of self-assembled heteropolymers. The soft reversible hydrogel is porous, but can be closed by small changes in environmental conditions such as $\mathrm{pH}$ or temperature. Such gels are abundant in any concentrated mixture of amphiphilic polymers, where in most cases the morphology is not a simple hollow sphere, but rather an intricate labyrinth of folded lamellae, tubes and droplets (van Vlimmeren et al. 1999). 


\section{Conclusion and future perspectives for research in Astrobiology}

The presence of life on Earth is directly related to the origin and evolution of the solar system and to the initial conditions present in the interstellar molecular cloud from which it condensed. Both the gas phase species listed in Table 1 and the less well characterized, but much heavier, organic solids in the ISM point towards the universality of organic chemistry. Taken together with the wide range of organic molecules found by laboratory analysis of carbonaceous meteorites, these results certainly suggest that the basic building blocks of life must be widespread in planetary systems in our Milky Way and other galaxies.

Life originated from a very complex carbon-based chemistry, which . probably resulted from the interaction of organic molecules and inert rocks. Extraterrestrial inorganic and organic materials (from comets, meteorites and interplanetary dust particles - IDPs) were and are continuously captured by the Earth (Chyba et al. 1990, Maurette \& Morbidelli 2001). Models on the cometary matter trapped by solar system objects have been developed showing the enormous $\left(10^{20}-10^{26} \mathrm{~g}\right)$ amount of carbon and silicon bearing materials brought by comets since planetary formation. These materials could contain prebiotic elements that found on the early Earth (or on other planetary surfaces) suitable physical and chemical conditions for the development of life.

Even if the ultimate source of these organic compounds turns out to be comets and meteorites, the recognition of their extraterrestrial origin does not imply by itself a reappraisal of panspermia, athough the possibility that terrestrial life actually began on Mars should not be ignored (e.g. Nisbet \& Sleep 2001). It represents rather the recognition that the primitive terrestrial environment was shaped at least in part by the intense bombardment that characterized the early evolution of the Earth and other solar system bodies. The link between cometary and interstellar matter remains unclear, with good correlation of ice content but puzzling results on silicates and deuterium content. The study of the delivery to Earth is itself still in its infancy: better models of the atmospheric entry of interplanetary particles are needed, to assess correctly the survival chances for molecules and enantiomeric excesses; the time dependence of the extraterrestrial flux on Earth must also be constrained.

Advanced technology in astronomical instrumentation and satellites will make major contributions to this research area in the following decades. Knowledge of the chemistry of dense interstellar clouds and protoplanetary disks will surely be significantly advanced by the higher sensitivity and angular resolution that will become available with new radio astronomy facilities such as the SMA (the sub-mm array, ALMA (the Atacama Large Millimeter Array, an interferometer of 64 antennas working between 30 and $900 \mathrm{GHz}$, in operation in 2010) and the Large Millimeter Telescope LMT. ALMA should be able to detect relatively complex prebiotic molecules, in particular in the part of protoplanetary disks where planetary formation is expected to take place, opening a new field of investigations. 
Space observatories such as FIRST-HERSCHEL and SIRTF will allow the study of molecules in stellar formation regions. The forthcoming generation of optical (such as the NGST) and submillimeter telescopes should allow the first quantitative studies of both dust and gas contents in molecular clouds and galaxies.

Meanwhile, we are forced to temper our elation at the long-anticipated discovery that ours is not the only planetary system in the galaxy with the realization that we still do not know if there are any other Earth-like planets. We need a new technique that can detect small planets like ours. The most promising approach at the moment is high precision photometry that would permit the detection of Earth-like planets passing in front of their stars as seen from Earth. The diminution of the light from the star will give the size of the planet, while the observation of successive transits will allow a precise determination of the orbit. To achieve the required photometric precision, it will be necessary to make the observations from a telescope in space: a goal of the COROT, KEPLER and EDDINGTON missions will be such photometric detection of Earth-like planets in other stellar systems. Statistics of exo-Jupiters will also constrain current models of star and planetary formation. In the 2015 time frame, nulling interferometers in space such as IRSI-DARWIN and TPF (Terrestrial Planet Finder) could permit us to measure the atmospheric spectra of terrestrial exoplanets, in particular of $\mathrm{CO}_{2}, \mathrm{CH}_{4}, \mathrm{H}_{2} \mathrm{O}$ and $\mathrm{O}_{3}$ traces.

Several important space probes are planned in the next years to investigate comets and asteroids. In addition to the ROSETTA in situ analysis by a lander and a 1-year orbiter, much is expected from the in situ cometary dust sample collection by STARDUST (to be returned 2006), and from several flyby or rendezvous missions (CONTOUR, DEEP IMPACT). With all these new studies one can hope to determine if comets have just brought some spice to the primitive soup, have brought essential components (water, carbon, chirality), or even have been by themselves a "complete kit" sufficient for life to start. This in turn will allow us to address more convincingly the question of the spread of life in our solar system and in other extrasolar systems. The Mars exploration programme will also permit us to study processes of geological and atmospheric evolution, as well as searches for extinct or extant life on Mars. In 2003 already the MARS-EXPRESS orbiter will perform studies of the water cycle, while the BEAGLE-2 lander will characterise surface conditions of exobiological relevance. Another target for astrobiology is the ocean of Europa below its icy crust.

Simulation of formation and processing of cosmic materials has allowed the identification of some of the carbonaceous matter present in space. Several astrophysical problems seem to have found a solution, but many others are still unsolved, and await further laboratory experiments to be understood. With the help of nanotechnology the missing carbon fraction in astronomical solids will be better defined. In such experimental programs, the search of links between materials present in different space environments and biogenic components are certainly a driver. A further focus for experiments is the catalytic effect of dust. Materials, such as Mg-rich olivine and pyroxene, synthesised and characterised in the laboratory (see section 3 ) could be used to study their potential role as catalysts for chemical reactions capable of providing viable routes for the formation of prebiotic materials. 
The chemistry of some prebiotic simulations is robust and supported by meteorite organic analyses, but the synthesis of biochemical constituents of contemporary organisms by non-enzymatic processes under laboratory conditions does not necessarily imply that they were either essential for the origin of life or available in the primitive environment. Understanding the constraints when defining the chemical composition of life's "raw material" from the viewpoints of different scientific disciplines is essential. This will help to describe the processes of self-organization that led to the establishment of the functional relationship between replicative polymers, energy-rich compounds and membrane-forming compounds, which eventually led to the first cells. Our existence is a consequence of the evolving universe. If nothing else - and there may be no further connection with the origin of life on Earth than this - the investigations of the ISM, the meteorites, the comets and the cold, dimly lit satellites of the outer solar system demonstrate the ease with which nature can assemble the organic compounds that play such an important role in the chemical evolution that preceded biological evolution. The step from prebiotic matter to life on Earth and possibly elsewhere is hidden in the mists of space and time. Penetrating those mists remains one of the grand adventures of science. It will surely continue to be enthusiastically pursued in the future.

\section{Acknowledgments:}

We acknowledge the support of the International Space Science Institute in Bern for hosting our working group: Prebiotic matter in space. We thank I. Ten Kate, Z. Martins and Z. Peeters for computer assistance and J. Crovisier for discussion.We would like to thank L. Allamandola, E. van Dishoeck, E. Gibb and Y. Pendleton, for authorizing the publication of some of their figures. We acknowledge support from NOVA (Dutch School of Astronomy), NASA grants NAG5-8718 and NAG5-9260 (Exobiology), NSF EAR-9753175, ASI and MIUR. A portion of this work was performed under the auspices of the U.S. Dept. of Energy at the University of California/Lawrence Livermore National Laboratory under contract no. W-7405-Eng48. 


\section{Figure captions}

Figure 1

Carbon, the $4^{\text {th }}$ most abundant molecule in the universe and the basis for organic chemistry, is produced by the triple- $\alpha$ process $\left(3 \times{ }^{4} \mathrm{He} \rightarrow{ }^{12} \mathrm{C}\right)$ in the cores of stars via nuclear fusion.

\section{Figure 2}

Observations of organic molecules towards $\mathrm{G} 327$ around $151.6 \mathrm{GHz}$, with suggested line identifications marked. The majority of the emission lines are from organic species such as $\mathrm{CH}_{3} \mathrm{OH}, \mathrm{CH}_{3} \mathrm{OCHO}, \mathrm{C}_{2} \mathrm{H}_{3} \mathrm{CN}, \mathrm{C}_{2} \mathrm{H}_{5} \mathrm{CN}$, and $\mathrm{CH}_{3} \mathrm{SH}$. Many of the dispayed lines are unidentified to date (taken from Gibb et al. 2000b). Intensity is given in the radio astronomy units of antenna temperature $\left(\mathrm{T}_{\mathrm{A}}{ }^{*}\right.$, in Kelvin).

\section{Figure 3}

The hypothesized basic structural and molecular character of carbonaceous, interstellar dust in the diffuse interstellar medium. The structure is a kerogen-type aromatic network bridged by aliphatic chains, including side-groups and heteroatoms. The specific geometries of the aromatic plates and aliphatic components are inferred. A tyical $0.1 \mu \mathrm{m}$ carbonaceous dust grain contains $\sim 10^{4}$ of such fragments. Encircled regions denote different structural units: (1) aromatic network, (2) aliphatic bridges, (3) aliphatic carbonyl groups, (4) aromatic carbonyl groups, (5) aromatic nitrogen groups (from Pendleton \& Allamandola 2001).

\section{Figure 4}

Fit to interstellar extinction curves from Cardelli \& Savage (1988) and Fitzpatrick \& Massa (1990) (dotted lines) by means of blends of the optical properties of UV processed carbon grains (solid lines). The Seaton average bump and the profiles with the extreme deviations observed in peak position and band width of the interstellar extinction bump are considered. HD38087 (minimum peak position) HD93028 (minimum width), and HD29647 (maximum peak position and width). Curves are up and down shifted by 1,2 , or 3 extinction scale units for the sake of clarity.

\section{Figure 5}

Comparison of (a) emission spectrum from the protoplanetary nebula IRAS $22272+5435$ with (b) the absorption spectrum from a mixture of neutral and cationic PAHs. Comparison of (c) emission spectrum from the Orion ionization ridge (d) the absorption spectrum from a mixture of fully ionized PAHs (from Allamandola et al. 1999). 


\section{Figure 6}

Mass absorption coefficients of natural Fe-rich olivine (FAYC - crystalline fayalite) and the same material after laser ablation (FAYA - amorphous fayalite). The other spectra refer to FAYA after thermal annealing at various temperatures (see top of each panel) and times (reported aside each plot). The spectra are shifted in ordinate for the sake of clarity (Brucato et al., 2002).

\section{Figure 7}

This figure summarizes the main stages leading to the formation of stars, showing the molecular species observed in the gas phase of a low-mass star: a) corresponds to the pre-stellar phase (also known as molecular cloud core); b) and c) correspond to the proto-stellar phases (YSO for Young Stellar Object); d) represents the T-Tauri phase (Pre-Main Sequence star) where a large disk of gas and dust is surrounding the young star. In e), the star has reached the Main-Sequence stage (starting its nuclear "burning") and is potentially surrounded by a planetary system (taken from van Dishoeck \& Blake 1998).

\section{Figure 8}

Solid state ${ }^{13} \mathrm{C}-\mathrm{NMR}$ spectrum of the insoluble organic matter from the Orgueil meteorite. Variable contact time and inversion recovery cross polarization along with magic angle spinning allowed the recognition of 8 different types of carbon, whose relative abundances could be determined through spectral deconvolution (Gardinier et al. 2000).

\section{Figure 9}

The water $\mathrm{D} / \mathrm{H}$ ratio is reported versus the organic matter $\mathrm{OM} \mathrm{D} / \mathrm{H}$ ratio. The two solid lines correspond to the kinetic co-evolution of the water and OM D/H ratios and can be regarded as "isotopic thermometers". The calculated temperatures for these two isotopic evolution pathways are 35 and $120 \mathrm{~K}$. The calculated high and positive energies ( $\Delta \mathrm{E}_{\mathrm{OM}}$ and $\Delta \mathrm{E}_{\mathrm{Water}}$ ) likely correspond to isotopic exchanges via ion-molecule reactions. This interpretation suggests that the deuterium enrichment process was "similar" in the whole solar system and the temperature of the isotopic exchange varied with heliocentric distance. Consequently, the young sun, in its YSO phase, seems to have governed the deuterium enrichment via an intense ionization of the surface of the protosolar nebula. 


\section{Figure 10}

The Urey-Miller experiment demonstrating the possible synthesis of biologically relevant molecules in an hypothesized primitive, reducing atmosphere. Water vapor, simulating heated gases from the Earth's interior, is passed through a chamber with gases such as $\mathrm{NH}_{3}, \mathrm{CH}_{4}$ etc. The heated gases were sparked with an electrode, simulating lightning; the molecules formed in the reaction were immediately cooled and then trapped in a collection chamber, simulating the oceans.

\section{Figure 11}

Isosurface representation of amphiphilic diblock copolymer microscopic hydrogel (80\% Poly Propylene Oxide - Poly Ethylene Oxide), predicted by simulation. The hydrogel (diameter ca. $80 \mathrm{~nm}$ ) is internally structured due to the microphase separation of hydrophobic (Poly Propylene Oxide) and hydrophilic (Poly Ethylene Oxide) block. Small changes in solvent quality will close or open the gel, thereby allowing release or uptake of reagents. The hydrogel resembles a $\mathrm{C}_{60}$ buckyball structure, but with a twist: the hydrogels are soft and fragile, not hard, and they are much larger. The design of such smart gels is an active area of research in drug delivery, with many topics of relevance to Artificial Cell programs. 


\section{Table captions}

Table 1

Interstellar and circumstellar molecules as compiled by $A$. Wootten (http://www.cv.nrao.edu/ awootten/allmols.html). Recent detections include benzene by Cernicharo et al. (2000). Larger molecules have not yet been unambigously identified, but there is no doubt that they are present.

Table 2

Relevant processes active in space and doses/rates in space and in laboratory (Colangeli et al. 1999).

\section{Table 3}

Molecules observed in the protoplanetary disk surrounding the TTauri star DM Tau. Both detections and upper limits at $3 \sigma$ are shown. Sv is given in Jy.km/s. " $\mathrm{f}$ " is the depletion factor in the gas phase with respect to $\mathrm{X}$, the abundance in TMC1 by comparison to $\mathrm{H}_{2}$. Abundances, measured in TMC-1, come from Ohishi et al. (1992) and Cernicharo \& Guelin (1987). For details, see Dutrey et al., (1997 and 2000).

\section{Table 4}

Molecular abundances of ices for comets Hyakutake and Hale-Bopp (in \% with respect to water ice). After Bockelée-Morvan et al. (2000) and Crovisier et al. (2002). The limits on $\mathrm{NaCl}$ and $\mathrm{NaOH}$ are still uncertain.

Notes

a) extended source - the abundance is model dependent

b) abundance deduced from CS

c) was measured $20 \%$ at 2,9 AU by the ISO satellite

\section{Table 5}

Abundances of soluble organic compounds in meteorites (taken from Botta et al. 2001). These data are measured in the Murchison CM2 meteorite (except aromatic hydrocarbons and fullerenes). Amino acids concentrations for several CI chondrites are also listed. a) average of the abundances in the CI carbonaceous chondrites Orgueil and Ivuna (Ehrenfreund et al., 2001a); b) for the Yamato-791198 carbonaceous chondrite (Naraoka et al., 1988); c) $0.1 \mathrm{ppm}$ estimated for $\mathrm{C}_{60}$ in Allende (Becker et al., 1994); d) Cooper \& Cronin 1995. 
Table 6

Modified from Chyba \& Sagan (1997). * assumes intermediate atmosphere

$\left[\mathrm{H}_{2}\right] /\left[\mathrm{CO}_{2}\right]=0.1$

I Synthesis of the Miller-Urey type

2 Such as that caused by lightning interacting with a volcanic discharge

3 An estimate for compounds created from the interaction between infalling objects and the Earth's atmosphere

4 Based on present-day estimates for total organic matter in hydrothermal vent effluent as reported by Kadko et al. (1994); cf., Elderfield \& Schultz (1996)

5 Conservative estimate based on possible cumulative input calculated assuming flux of $10^{22} \mathrm{~kg}$ comets during first $10^{9} \mathrm{yr}$ of Earth's history. If comets contain on the order of $15 \mathrm{wt} \%$ organic material (e.g., Delsemme, 1992), and if $\approx 10 \%$ of this material survives, it will comprise approximately $10^{11} \mathrm{~kg} / \mathrm{yr}$ average flux via comets during the first $10^{9}$ yr. IDP's are by far more easy to deliver; comets do have certainly more volatiles and are enriched in carbon and enter in the Earth's atmosphere very fast. The main source of extraterrestrial water and organics is still debated.

\section{Table 7}

$\mathrm{D} / \mathrm{H}$ ratios of water and organic matter in the solar system reported in units of $10^{-6}$.

Notes (1) OM : Organic matter. In meteorites SOM and IOM referred to as soluble and insoluble, respectively (2) Interstellar Medium: dense molecular clouds (3) Domain defined for different initial fractions of atomic D and different "condensed solid" to gas ratio. (4) Domain defined for different organic molecules. (5) Mostly measured in HCN. (6) Four independent spectroscopic determinations on 3 comets. (7) OM as HCN in Hale-Bopp in HCN (8) From SNC meteorites. (9) The D/H ratio in meteoritic water is calculated by mass balance using $\mathrm{C}$ and $\mathrm{H}$ concentrations and $\mathrm{D} / \mathrm{H}$ ratios, determined in whole rock and IOM samples. (10) Error bars stands for 1 $\sigma$ calculated on mean values averaged from published data. (11) Maximum values corrected for solvent contamination during SOM extractions. (12) Water D/H ratio cannot be calculated but must be lower than that of the whole rock $\mathrm{D} / \mathrm{H}$ ratio; hence the sign $\leq$. (13) Clays in Semarkona range from 85 to $720 \times 10^{-6}$. (14) Two deuteriumrich organic compounds where identified in this IDP with $\mathrm{D} / \mathrm{H}=250$ and $150010^{-6}$; hence the large error bars. (15) Calculated assuming $\mathrm{C} / \mathrm{H}$ in organics $=1$ and a $\mathrm{C} / \mathrm{H}$ ratio in the bulk comet between 0.4 and 0.6 .

References : [ 1 ]: Brown \& Millar (1989). [ 2 ]: Guélin et al., (1982). Millar et al., (1989). [ 3 ]: Jacq et al., (1990). [ 4 ]: Gensheimer et al., (1996). [ 5 ]: Hatchell et al, (1998). [ 6 ]: Schilke et al, (1992). [ 7 ]: Meier et al., (1998). [ 8 ]: Balsiger et al., (1995). Eberhardt et al., (1987). Bockelée-Morvan et al., (1998). [ 9 ]: Leshin (2000). [ 10 ] Lécuyer (1998). [ 11 ]: Becker R. H. \& Epstein S. (1982). Boato G. (1954). Deloule E. \& Robert F. (1995). Deloule, E. et al., (1998). Epstein et al., (1987). Halbout et al., (1990). Kerridge (1985). Kerridge et al., (1987). Krishnarnurthy et al., (1992). Pizzarello et al., (1991). Mc Naughton et al., (1981). Mc Naughton et al., (1982). Robert et al., (1979). Robert F. and Epstein S. (1982). Robert et al., (1987). Sears et al., (1995). Yang J. \& Epstein S. (1983). Yang J. \& Epstein S. (1984). McKeegan et al., (1985). Zinner et al., (1983). [ 12 ] Messenger \& Walker (1998), Messenger S. (2000) Aléon et al. (2002) [ 13 ] Geiss \& Gloecker (1998). 


\section{References}

A'Hearn MF, Millis RL, Schleicher DG, Osip DJ, Birch PV 1995 Icarus 118 223-70

Aléon J, Engrand C, Robert F and Chaussidon M 2002 Geochim Cosmochim Acta (in press)

Allamandola LJ, Hudgins DM, Sandford SA. 1999 Astrophys. J. 511:L115-19

Altwegg K, Balsinger H, Geiss J 1999 ISSI Space Science Reviews 90 3-18

Anders E. 1989 Nature 342 255-57

André Ph and Montmerle T 1994 Astrophys J 420 837-862

Bailey J, Chrysostomou A, Hough JH, Gledhill TM, McCall A, Menard F and Tamura M 1998 Science 281 672-674

Balsiger H, Altwegg K and Geiss J 1995 JGeophys Res 100 5827-5834

Bandurski EL and Nagy B 1976 Geochim Cosmochim Acta 40 1397-1406

Becker L, Poreda RJ and Bunch TE 2000 Proc Natl Acad Sci USA 97 2979-2983

Becker L, Poreda RJ, Hunt AG, Bunch TE and Rampino M 2001 Science 291 15301533

Becker RH and Epstein S 1982 Geochim Cosmochim Acta 46 97-103

Binet L, Gourier D, Derenne S and, Robert F Geochim Cosmochim Acta, submitted

Bitz SrMC and Nagy B 1966 Proc Nat Acad Sci 56 1383-1390

Biver, et al 1997 published 1999 Earth, Moon and Planets 78 5-11

Blank JG and Miller GH 1998 Proceedings of the 21st International Symposium on Shock Waves 1467-1472

Blank J, Miller G, Ahrens MJ, Winans RE 2001 OLEB 31 15-51

Boato G. 1954 in The isotopic composition of hydrogen and carbon in the carbonaceous chondrites. 6 209-220

Bockelée-Morvan D 1997 Molecules in Astrophysics: Probes and Processes, IAU symposium 178219

Bockelée-Morvan D, Gautier D, Lis DC, Young K, Keene J, Phillips T, Owen T, Crovisier J, Goldsmith P F Bergin E A Despois D and Wootten A 1998 Icarus Volume 133 Issue 1 147-162 
Bockelée-Morvan D, Lis DC, Wink JE, Despois D, Crovisier J, Bachiller R, Benford DJ, Biver N, Colom P, Davies JK, Gérard E, Germain B, Houde M, Mehringer D, Moreno R, Paubert G and Phillips TG, Rauer H 2000 Astron.Astrophys. 353110

Bonner WA 1991 Orig Life Evol Biosph 21 59-111

Bonner WA, Greenberg JM, Rubenstein E 1999 OLEB 29 215-219

Botta O, Glavin DP Bada JL 2001 Meteoritics \& Planetary Space Science 36, 26

Brown PD and Millar TJ 1989 Monthly Notices Roy Astron Soc 237 661-671

Brown PD and Charnley SB 1990 Monthly Notices Of The Royal Astronomical Society Jun V244(N3):432-443

Brown RD, Rice E 1981 Royal Society, Philosophical Transactions, Seris A, 303 $1480523-533$

Brucato JR, Colangeli L, Mennella V, Palumbo P, Bussoletti E 1999

Astron. Astrophys. 348, $1012-1019$

Brucato JR, Mennella V, Colangeli L, Rotundi A, Palumbo P. 2002, PSS, submitted

Bunch TE, Chang S 1980 Geochim. Cosmochim. Acta 44 1543-1577

Cardelli J, Meyer DM, Jura M, Savage BD. 1996 Astrophys. J. 467:334 40

Cardelli JA, Savage BD 1988 Astrophys.J. 325864

Cassen P 1994 Icarus 112 405-429

Ceccarelli C, Vastel C, Tielens AGGM, Castenets A, Boogert ACA, Loinard L \& Caux E 2002 AA 381 L17-L20

Cernicharo J and Guélin M 1987 AA 176299

Cernicharo J 2000 Astrochemistry: From Molecular Clouds to Planetary Systems 375-392

Cernicharo J, Heras A, Tielens AGGM, Pardo JR, Herpin F, Guelin M, Waters LBFM 2001 Astrophys.J. 546 L123-126

Charnley SB, Ehrenfreund P, Kuan YJ. 2001 Acta Spectrochimica 57 685-704

Charnley SB, Rodgers SD, Kuan YJ, Huang HC 2002, Adv. Space Res. (in press)

Chiang EI, Joung MK, Creech-Eakman MJ, Qi C, Kessler JE, Blake GA and van Dishoeck EF 2001 Astrophys J 547 1077-1089 
Chyba CF, Thomas PJ, Brookshaw L and Sagan C 1990 Science 249 366-373

Chyba CF and Sagan C 1992 Nature 355 125-132

Chyba CF and Sagan C 1997 Comets and the Origin and Evolution of Life 147-173

Clark BC, 1987 Icarus $71250-256$

Clark BC, 1988 Origins of Life and Evolution of the Biosphere 18 209-238

Cody GD III , Alexander CMO'D and Tera F 1999 Meteor Planet Sci 34 A25-A26

Colangeli L, Mennella V, Palumbo P, Rotundi A and Bussoletti E. Astron. Astrophys. Suppl. 113, $561-577$

Colangeli L, Mennella V, Brucato JR, Palumbo P, Rotundi A 1999 Space Science Reviews 90, Issue 1-2 341-354

Cooper GW, Cronin JR: 1995 Geochim. Cosmochim. Acta 59, 1003-1015

Cooper GW, Thiemens MH, Jackson TL and Chang S 1997 Science 277 1072-1074

Cooper GW, Horz F, Oleary A anc Chang S 2000 Lunar Planet Sci Conf 31 3232-3

Cooper GW, Kimmich N, Belisle W, Sarinana J, Brabham K and Garrel L 2001 Nature 414 879-883

Cottin H, Gazeau MC, Benilan Y and Raulin F 2001 Astrophys J 556 417-420

Cronin JR, Pizzarello S and Frye JS 1987 Geochim Cosmochim Acta 51 299-303

Cronin JR and Chang S 1993 The Chemistry of Life's Origin 209-258

Cronin JR and Pizzarello S 1997 Science 275 951-955

Cronin JR, Pizzarello S and Cruikshank DP 1998 Meteorites and the Early Solar System 819-857

Crovisier J, Leech K, Bockelee-Morvan D., Brooke TY, Hanner MS, Altieri V, Keller HU and Lellouch E. 1997 Science 275, 1904 - 1907

Crovisier J. 1997 published 1999 Earth,Moon and Planets 79, 125143.

Crovisier J 1998 in Astrochemistry: From Molecular Clouds to Planetary systems IAU197, eds. YC Minh \& E. van Dishoeck ASP $461-470$

Crovisier J \& Bockelee-Morvan D 1999 Space Science Rev. 90 19-32 
Crovisier J. and Encrenaz Th. (2000)

Comet Science. The Study of the Remnants from the Birth of the Solar System.

Crovisier J, Bockelee-Morvan D, Colom P, Biver N, Despois D, Lis DC, Benford DJ, Mehringer D et al. (2002) The composition of ices in comet C/1995 O1 (HaleBopp): Upper limits on undetected species from radio spectroscopy, Astronomy and Astrophysics. , in prep.

Curl RF 1993 Phil Trans R Soc Lond A 343 19-32

D'Alessio P, et al 1999 ApJ 527893

Dartois E, Schutte WA, Geballe TR, Demyk K, Ehrenfreund P, et al. 1999 Astron. Astrophys. 342:L32--35

Dartois E, d'Hendecourt L. 2001 Astron \& Astrophys. 365 144-156

De Duve C 1991 The Nature and Origin of Life

de Vries MS, Reihs K, Wendt HR, Golden WG, Hunziker HE, Fleming R, Peterson E and Chang S 1993 Geochim Cosmochim Acta 57 933-938

Degens ET and Bajor M 1962 Naturwissenschaft 49 605-606

Deloule E and Robert F 1995 Geochim Cosmochim Acta 59 4695-4706

Deloule E, Doukhan J-C and Robert F 1998 Geochim Cosmochim Acta 62 33673378

Delsemme AH 1992 Orig Life Evol Biosphere 21 279-298

Delsemme AH 2000 Icarus 146 313-325

Despois D 19971999 Earth Moon and Planets 79 103-124

d'Hendecourt L, Jourdain de Muizon M, Dartois E, Breitfellner M, Ehrenfreund P, et al. 1996. Astron. Astrophys. 315:L365--68

Dickens JE and Irvine WM, Snell RL, Bergin EA, Schloerb FP, Pratap P and Miralles MP 2000 Astrophys J 542870

Dickens JE and Irvine WM, Ohishi M, Ikeda M, Ishikawa S, Nummelin A and Hjalmarson Å 1997 Astrophys J 489753

Drouart A and Dubrulle B, Gautier D and Robert F 1999 Icarus 140 129-155

Dubrulle B 1992 a Astron Astrophys 266 592-604 
Duchesne J, Depireux J and Litt C 1964 Geoch Int 1 1022-1024

Dutrey A, et al 1994 AA 286149

Dutrey A, Guilloteau S and Guélin M 1997 Astronomy \& Astrophysics 31 L55-58

Dutrey A 1999 Proceedings from Planets outside the Solar System: Theory and Observations 13-49

Dutrey A, et al 2000 Proceedings of IAU symposium 197 415-424

Dwek E. 1997 Astrophys. J. 484:779--84

Dyson F 1985 Origins of Life

Ebel, D.S.: 2000, JGR 105, 10363

Eberhardt P, Dolder U, Schulte W, Krankowsky D, Lammerzal P, Hoffmann JH, Hodges RR, Berthelier JJ and Illiano JM 1987 Astron Astrophys 187 435-437

Ehrenfreund P, Robert F, d'Hendecourt L and Behar F 1991 Astron Astrophys 252 712-717

Ehrenfreund P, Breukers R, D'Hendecourt L, Greenberg JM 1992a Astron. Astrophys. $260431-436$

Ehrenfreund P, Robert F and d'Hendecourt L 1992b Adv Space Res 12 (4)53-(4)56

Ehrenfreund P, Boogert A, Gerakines P, Tielens AGGM, van Dishoeck E. 1997 Astron \& Astrophys 328, 649-669

Ehrenfreund P, Schutte WA 2000, In Astrochemistry: From Molecular Clouds to Planetary Systems, IAU Symposium 197, ed. Y.C. Minh, E.F. van Dishoeck, pp. 135-46, Sogwipo:Astron. Soc. Pac.

Ehrenfreund P and Charnley SB 2000 Ann Rev Astron Astrophys 38 427-83

Ehrenfreund P, Glavin DP, Botta O, Cooper G and Bada JL 2001a PNAS 98 21382141

Ehrenfreund P, Bernstein MP, Dworkin JP, Sandford SA, Allamandola LJ 2001b Astroph. J. 550 95-99

Eigen M and Schuster P 1979 The Hypercycle - A Principle of Natural SelfOrganization

Elderfield H and Schultz A 1996 Ann Rev Earth Sci 24 1919-224 
Ellder J, Friberg P, Hjalmarson Å, Höglund B, Irvine WM, Johansson LEB, Olofsson H, Rydbeck G, Rydbeck OEH and Guélin M 1980 Astrophys J (Lett) 242 L93

Engel MH, Macko SA 1997 Nature 389, 265-268

Epstein S, Krishnamurthy RV, Cronin JR, Pizzarello S and Yuen GU 1987 Nature $326477-479$

Fabian D, Jäger C, Henning Th, Dorschner J and Mutschke H 2000 Astron. Astrophys, 364, $282-292$

Festou MC, Rickman H and West RM 1993a Astronomy and Astrophysics Review 4 363-447

Festou MC, Rickman H and West RM 1993b Astronomy and Astrophysics Review 5 $37-163$

Feuchtgruber H, Helmich FP, van Dishoeck EF and Wright CM 2000 Astrophys J $535 \mathrm{~L} 111$

Fitzpatrick EL and Massa D 1990 Astrophys. J. Suppl. 72, 163 - 189

Flynn GJ, Keller LP, Jacobsen C and Wirick S 2001 Meteoritics and Plan. Sci. 34 A36

Fomenkova MN, Chang S and Mukhin LM 1994 Geochimica et Cosmochimica Acta 58 4503-4512

Fomenkova MN 1999 Space Science Reviews 90 109-114

Fox SW 1988 The Emergence of Life: Darwinian Evolution from the Inside

Frenklach M, Feigelson ED 1989 Astrophys 317 L59-L62

Gardinier A, Derenne S, Robert F, Behar F, Largeau C and Maquet J 2000 Earth Planet Sci Letters 184 9-21

Gauthier P, Hersant F, MousisO, Lunine J 2001a, ApJ 550 L227

Gauthier P, Hersant F, MousisO, Lunine J 2001b, ApJ 559 L183

Geiss J, Gloecker G 1998 Space Science Rev 84 239-250

Gensheimer PD, Mauersberger R and Wilson TL 1996 Astron Astrophys314 281294

Gesteland RF, Cech TR, Atkins JF (eds), The RNA World, Cold Spring Harbor Laboratory Press, New York, 1999 
Gibb E, Whittet DCB, Schutte WA, Chiar J, Ehrenfreund P, et al. 2000a. Astrophys. J. 536 347-56

Gibb E, Nummelin A, Irvine WM, Whittet DCB and Bergman P 2000b Astrophys J 545309

Gibb EL, Whittet DCB, Chiar JE 2001 Astroph. J. 558 702-716

Gibbons RV and Ahrens TJ 1971 Journal of Geophysical Research 76 5489-5498

Glassgold A, Feigelson ED and Montmerle T 2000 Protostars and Planet IV 429-455

Greenberg JM 1996 Physical origin of homochirality in life, Santa Monica, California February 1995185

Greenberg J. M. (1982): What are comets made of? A model based on interstellar dust. In Comets, ed. Wilkening L. L., University Arizona Press, Tucson, pp. 131 $163,1982$.

Grossman, L.: 1972, Geochim. Cosmochim. Acta 38, 47

Guélin M, Langer WD and Wilson RW 1982 Astron Astrophys 107 107-127

Guélin M, Lucas R, Neri R, Bremer M and Broguiere D 2000 Astrochemistry: From Molecular Clouds to Planetary Systems 365-374

Halbout J, Robert F and Javoy M 1990 Geochim Cosmochim Acta 54 1453-1462

Hallenbeck S. L., Nuth III J. A. and Daukantas PL. 1998 Icarus $131198-309$

Hansen CJ and Kawaler SD 1994 Stellar Interiors: physical principles, structure and evolution, Springer Verlag

Hartquist TW and Williams DA 1995 The Chemical Controlled Cosmos, Astronomical Molecules from the Big Bang to Exploding stars, Clarendon Press, Oxford

Hatchell J, Millar TJ and Rodgers SD 1998 Astron Astrophys 332 695-703

Hayatsu R, Matsuoka S, Scott RG, Studier MH and Anders E 1977 Geochim Cosmochim Acta 41 1325-1339

Hayatsu R, Winans RE, Scott RE, McBeth RL, Moore LP and Studier MH 1980 Science 207 1202-1204

Hayes JM 1967 Geochim Cosmochim Acta 31 1395-1440

Herbst E, Adams NG and Smith D 1983 Astrophys J 269 329-333 
Herbst E 2000 in Astrochemistry: From Molecular Clouds to Planetary Systems, IAU197, eds. YC Minh \& E. van Dishoeck ASP 147-160

Herlin N, Bohn I, Reynaud C, Cauchetier M, Galvez A, Rouzaud, JN 1998, A\&A 330,1127

Huber C and Wächtershäuser G 1998 Science 281 670-672

Huebner WF 1987 Science 237 628-630

Irvine WM and Knacke RF 1989 Origin and Evolution of Planetary and Satelite Atmospheres 3-34

Irvine WM, Dickens JE, Lovell AJ, Schloerb FP, Senay M, Bergin EA, Jewitt D and Matthews HE 1998a Faraday Disc 109475

Irvine WM, Dickens JE, Lovell AJ, Schloerb FP, Senay M, Bergin EA, Jewitt D and Matthews HE 1998b Nature 393 547-550

Irvine WM 1999 Space Sci Rev 90203

Irvine WM and Bergin EA 2000 Astrochemistry: From Molecular Clouds to Planetary Systems IAU197, eds. YC Minh \& E. van Dishoeck ASP 447-460

Irvine WM, Schloerb FP, Crovisier J, Fegley B Jr and Mumma MJ 2000 Protostars and Planets IV 1159- 1200

Jacq T, Walmzey CM, Henkel C, Baudry R, Mauersberger R and Jewell PR 1990 Astron Astrophys 228 447-458

Jull AJT, Beck JW, Burr GS, Gilmour IA, Sephton MA and Pillinger CT 1999 Meteor Planet Sci 34 A60

Kadko D, Baker E, Alt J and Baross J 1994 Final Rept RDGE/VENT workshop 55

Kawakita H, Watanabe J, Ando H et al. 2001 Science 294 1089-1091

Keane J, Tielens AGGM, Boogert ACA, Schutte WA, Whittet DCB 2001a Astron \& Astrophys 376, 254-270

Keller LP, Messenger S and Bradley JP 2000 JGR 10510397

Kerridge JF 1985 Geochim Cosmochim Acta 49 1707-1714

Kerridge JF, Chang S and Shipp R 1987 Geochim Cosmochim Acta 51 2527-2540

Khare BN, Thompson WR, Sagan C, Arakawa ET, Meisse C, Gilmour I 1990 NASA CP-3077 340-356

Kissel J and Krueger FR 1987 Nature $326755-760$ 
Kissel J, Krueger FR, Roessler K 1997 Comets and the Origins and Evolution of Life 69-110

Koerner D, et al 1993 Icarus 2106

Koike C, Nakagawa M, Koike K, Okada M, Chihara H, Tsuchiyama A 2001, Meteoritics \& Planetary Science 36, 101

Komiya M, Shimoyama A, Harada K 1993 Geochim Cosmochim Acta 57 907-914

Krankowsky D 1991 Comets in the post-Halley era Vol 2 855-877

Krishnarnurthy RV, Epstein S, Cronin JR, Pizzarello S, Yuen GU 1992 Geochim Cosmochim Acta 56 4045-4058

Kvenvolden K, Lawless J, Pering K, Peterson E, Flores J, Ponnamperuma C, Kaplan IR and Moore C 1970 Nature 228 623-626/ 923-926

Lacy JH, Faraji H, Sandford SA, Allamandola LJ 1998 Astroph. J. 501 L105-L108

Lagrange A-M, et al 2000 Protostars and Planets IV 639

Langer WD, van Dishoeck EF, Bergin EA, Blake GA, Tielens AGGM, Velusamy T, Whittet DCB 2000 Protostars and Planets IV 29-58

Lécluse C and Robert F 1994 Geochim Cosmochim Acta 58 2297-2939

Lécuyer C, Gillet Ph,Robert F 1998 Chemical Geology 145 249-261

Lequeux J, 2000 JGR 105, 10249-10256

Leshin LA 2000 Geophys Res Lett 27 14, 2017-2020

Levy RL, Grayson MA and Wolf CJ 1973 Geochim Cosmochim Acta 37 467-483

Lin DNC \& Ido S 1997 ApJ 477 781-791

Lin DNC \& Papaloizou JBC 1986 ApJ 309 846-857

Lunine J, Engel S, Rizk B, Horanyi M 1991 Icarus 94 333-344

MacKinnon IDR and Rietmeijer FJM 1987 Rev Geophys 251527

Malfait K, Waelkens C, Waters LBFM., Vandenbussche B, Huygen E and De Graauw MS 1998 Astron. Astrophys 332, L25 - L28

Marcy G, Cochran W, Mayor M, 2000, in Protostars and Planets IV, eds. V. Mannings, A. Boss, S. Russell, Univ. of Arizona Press, Tucson, 1285-1312 
Matteucci F 1991 Chemistry in Space, NATO Proc. 323 3-42

Maurette M, Duprat J, Engrand C, Gounelle M, Kurat G, Matrajt G and Toppani A $20001117-1137$

Maurette M and Morbidelli A 2001 M\&PSA 36127

Mayor M, \& Queloz D. 1995 Nature 378355

Mc Naughton NJ, Borthwicks S, Fallick AK and Pillinger CT 1981 Nature 294 639641

Mc Naughton NJ, Fallick AE and Pillinger CT 1982 Journal of Geophysical research 87 a294-a302

McKay CP and Borucki WJ 1997 Science 276 390-392

McKay DS, Gibson EK, Thomas- Keprta KL, Vali H, Romanek CS, Clemett SJ, Chillier XDF, Maechling CR and Zare RN 1996 Science 273 924-930

McKeegan KD, Walker RM and Zinner E 1985 Geochim Cosmochim Acta 49 1971-1987

Meier R, Owen TC, Jewitt DC, Matthews HE, Senay M, Biver N, Bockelée-Morvan D, Crovisier J and Gautier D 1998 Science Vol 279 1707-1710

Melosh HJ and Vickery AM 1989 Nature $338 \quad 487-489$

Mennella ., Colangeli L, Blanco A, Bussoletti E, Fonti S, Palumbo P. and Mertins HC 1995 Astrophys. J. $444288-292$

Mennella V, Colangeli L, Palumbo P, Rotundi A, Schutte WA and Bussoletti E. 1996 Astrophys. J. Lett. 464 L191 - L194

Mennella V, Baratta G.A, Colangeli L, Palumbo P, Rotundi A, Bussoletti E. and Strazzulla G. 1997 Astrophys. J. 481545 - 549

Mennella V, Colangeli L, Bussoletti E, Palumbo P. and Rotundi A. 1998 Astrophys. J. Lett. 507 L177 - L180

Mennella V., Brucato J. R., Colangeli L. and Palumbo P. 1999 Astrophys. J. Lett. 524 L71 -L74

Mennella V, Munoz Caro GM, Ruiterkamp R, Schutte WA, Greeenberg JM, Brucato J. R., Colangeli L. 2001 Astron. Astrophys. 367, 355-361

Messenger S, and Walker RM 1998 in Astrophysical implications of the laboratory study of presolar materials. eds Bernatowicz, T.J. \& Zinner, E. AIP Conf. Proc. 402, Am. Inst. Phys., Woodbury, New York, 545-564

Messenger S 2000 Nature 404 968-971 
Messenger S. 2001 Meteoritics \& Planetary Science 36133

Millar TJ, Bennett A and Herbst E 1989 Astrophys J 340 906-920

Millar TJ 1997 Molecules in Astrophysics: Probes and Processes 75-88

Millar TJ, Macdonald GH and Gibb AG 1997 Astronomy And Astrophysics 325

$1163-1173$

Miller GH 1998 Icarus 134 163-175

Miller SL and Urey HC 1953 Science $130245-251$

Miller SL and Lazcano A 2002 Proceedings of the Gold Medal Symposium on the Origin of Life(in press)

Mojzsis SJ, Ahrrenius G, McKeegan KD, Harrison TM, Nutman AP and Friend CRL 1996 Nature 384 55-59

Mojzsis SJ, Harrison TM and Pidgeon RT 2001 Nature 409 178-181

Molster FJ, Yamamura I, Waters LBFM, et al. 1999 Nature 401, 563-565

Moreels G, Clairemidi J, Hermine P, Brechignac P and Rousselot P 1994

AstronAstrophys 282 643-656

Mousis O, Gautier D, Bockelée-Morvan D, Robert F, Dubrulle B and Drouart A 2000 Icarus 148 513-525

Mumma MJ et al. 2001, Ap.J. 546, 1183

Murae T 1994 Proc NIPR Symp Antarct Meteorites 7 262-274

Najita J, et al 2001 ApJ 561880

Naraoka H, Shimoyama A, Komiya M, Yamamoto H, Harada K. 1988 Chem. Lett., 831-834

Newburn RL, Neugebauer M, Rahe J 1991 Comets in the ost-Halley Era Vol. 1\& 2

Nisbet E, Sleep N 2001 Nature 4091083

Nummelin A, Bergman P, Hjalmarson $\AA$, Friberg P, Irvine WM, Millar TJ, Ohishi M and Saito S 2000 Astrophys J Suppl 128213

Nuth JA, Hallenbeck SL and Rietmeijer FJM. 2000a J. Geophys. Res. 105, 10,387 10,396

Nuth JA, Hill HGM and Kletetschka G 2000b Nature $406275-276$ 
Pendleton YJ, Sandford SA, Allamandola LJ, Tielens AGGM. and Sellgren K. 1994 Astrophys. J., $437683-696$

Papoular R, Conard J, Guillois O, Nenner I, Reynaud C, Rouzaud JN 1996 Astron. \& Astrophys. 315, 222-236

Ohishi M, Irvine W, Kaifu N 1992 Proceedings IAU symp 150, ed. PD Singh Kluwer Academ. Publ, Dortdrecht 171

Oró J 1961 Nature 190 389-390

Owen T, Bar-Nun A. 1995 Icarus $116215-226$

Owen T 2000 ESA SP-451 163-169

Owen T, Mahaffy P, Niemann H, Atreya S, Donahae T, Bar-Nun A, de Peter I 1999, Nature $402 \quad 269-270$

Palumbo ME, Geballe TR, Tielens AGGM, et al. 1997

Astrophys. J. 479:839--44

Palumbo ME, Tielens AGGM, Tokunaga AT. 1995.

Astrophys. J. 449:674-80

Pendleton YJ, Allamandola LJ 2002 Astroph. J. Suppl. 138 75-98

Peterson E, Horz F and Chang S 1997 Geochimica et Cosmochimica Acta 61 39373950

Pierazzo E and Chyba CF 1999 Meteoritics \& Planetary Science 34 909-918

Pillinger CT 1998 Meteorit Planet Sci 33 603-622

Pizzarello S, Krisnamurthy RV, Epstein S and Cronin JR 1991 Geochim Cosmochim Acta 55 905-910

Pizzarello S, Huang Y, Becker L, Poreda RJ, Nieman RA, G Cooper and Williams M 2001 Science 293 2236-2239

Pratap P, Dickens JE, Snell RL, Miralles MP, Bergin EA, Irvine WM and Schloerb FP 1997 Astrophys J 486862

Prialnik D, Podolak M, 1999 Space Science Reviews, 90 (1-2): 169-178

Queloz D 2000 ESA SP-451 31-37

Robert F, Merlivat L and Javoy M 1979 Nature 282 785-789

Robert F and Epstein S 1982 Geochim Cosmochim Acta 16 81-95 
Robert F, Gautier D and Dubrulle B 2000 Space Science Reviews 92 201-224

Robert F 2001 Science 293 1056-1058

Robert F 2002 Proceedings of the Meudon Symposium 2001 : Deuterium in the Univers, Planet Space Science (in press)

Robert FM, Javoy J, Halbout B, Dimon B and Merlivat L 1987 Geochim

Cosmochim Acta 51 1787-1806

Roberts H and Millar TJ 2000 Astron Astrophys 361388

Rodgers SD and Millar TJ 1996 Mon Not Royal Astron Soc 280 1046-1054

Rodgers SD and Charnley SB 1998 Astrophys. J. Lett 501 L207-210

Rodgers SD and Charnley SB 2001 MNRAS 32384

Roueff E, Tiné S, Coudert LH, Pineau des Forêts G, Falgarone E and Gerin M 2000 Astron Astrophys 354 L63

Rubenstein E, Bonner WA, Noyes HP, Brown GS 1983 Nature 300118

Sakata A, Wada S, Tanabé T, Onaka T 1984, ApJ 287, L51

Saladino R, Mincione E, Crestini C, Costanzo G, Negri R and Di Mauro E 1996 J Am Chem Soc 1185615

Saladino R, Crestini C, Costanzo G, Negri R, Di Mauro E 2001 Bioorg Med Chem 9 1249

Salisbury JW, d'Aria DM and Jarosewich E 1991 Icarus 92 280-297

Sandford SA and Walker RM 1985 ApJ 291838

Sandford SA, Allamandola LJ 1993 Astroph. J. 409 65-68

Sandford SA, Bernstein MP, Allamandola LJ, Goorvitch D., Teixeira TCVS 2001, Astrophys. J. 548, 836

Schilke P, Walmsley CM, Pineau des Forêts G, Roueff E, Flower DR and Guilloteau S 1992 Astron Astrophys 256 595-612

Schnaiter M, Mutschke H, Henning Th, Lindackers D, Strecker M, Roth P 1996, ApJ 464, L187

Schopf JW 1993 Science $260640-646$

Schulz KF and Elofson RM 1965 Geochim Cosmochim Acta 29 157-160 
Sears DWG, Morse AD, Hutchison R, Guimon KR, Kie L, Alexander CMO'D, Benoit PH, Wright I, Pillinger CT, Kie T and Lipschutz ME 1995 Meteoritics 30 169-181

Segré D and Lancet D 2000 EMBO Reports11(31) 217-222

Segré D, Ben-Eli D, Deamer DW and Lancet D 2001 Origins of Life and Evolution of the Biosphere 31(1-2) 119-145

Sephton MA, Pillinger CT and Gilmour I 1998 Geochim Cosmochim Acta 62 18211828

Sephton MA, Pillinger CT and Gilmour I 1999 Planetary and Space Science 47 181187

Sephton MA, Pillinger CT and Gilmour I 2000 Geochim Cosmochim Acta 64 321328

Sephton MA and Gilmour I 2001 Planet Space Sci 49 465-471

Shu FH 1982 The Physical Universe, University Science Books (Sausalito, CA).

Shull JM 1987 Interstellar Processes ed. D. Hollenbach et al. 225-244

Snow TP and Witt AN 1995 Science $2701455-1457$

Snow TP and Witt AN 1996 Astrophys. J. Lett. 468, L65 - L68

Snyder DE 1997 Origins of Life and Evolution of the Biosphere 27 115-133

Steel D 1992 Origins of Life and Evolution of the Biosphere 21 339-357

Strazzulla G, Baratta GA and Palumbo ME 2001 Spectrochim Acta 57/4 825-842

Studier MH, Hayatsu R and Anders E 1972 Geochim Cosmochim Acta 36 189-215

Sugita S and Schultz P 2001 Amer Chem Soc Abstr 221 170-GEOC

Szostak JW, Bartel DP and Luisi PL 2001 Nature 409 387-390

Texeira TC, Devlin GP, Bush G and Emerson JP 1999 Astrophys J 347 L19-L22

Thiemann W, Meierhenrich U 2001, OLEB 31 1/2 199-210

Thomas PJ and Brookshaw L 1997 Numerical models of comet and asteroid impacts $131-145$ 
Tielens AGGM, Whittet DCB. 1997. In Molecules in Astrophysics:

Probes and Processes, ed. EF van Dishoeck, 45--60. Dordrecht:

Kluwer

Tielens AGGM, Hony S, van Kerckhoven C, Peeters E. 1999.

In: The Universe as seen by ISO ESA SP 427:579--88.

Tingle TN, Tyburczy JA, Ahrens TJ and Becker CH 1992 Origins of Life and

Evolution of the Biosphere 21 385-397

Trimble V 1997 Origins Life Evol Biosphere 273

Turner BE 1990 Astrophys J 362 L29

Vandenabeele-Trambouze O, Dobrijevic M, Despois D, Commeyras A et al 2001 Frontiers of Life, Acte des XIleme recontres de Blois, in press

Vandenbussche B, Ehrenfreund P, Boogert ACA., Dishoeck EF, Schutte WA. et al. 1999, Astron. Astrophys. 346, L57-L60

van Dishoeck EF and Blake G Ann. Rev. Astron.Astrophys. 36317

van Dishoeck EF 1998 in The Molecular Astrophysics of Stars and Galaxies, ed. TW Hartquist \& DA Williams, Clarendon Press, Oxford 53

Van Vlimmeren BAC, Maurits NM, Zvelindovsky AV, Sevink GJA and Fraaije JGEM 1999 Macromolecules 32(3) 646-656

Vidal-Madjar A, et al 1998 P\&SS 46629

Weissmann P. 1999 Space Science Reviews 90 301-11

Whittet DCB, Boogert ACA, Gerakines PA, Schutte W, Tielens AGGM, de Graauw Th, Prusti T, van Dishoeck EF, Wesselius PR and Wright CM. 1997 Astrophys. J., $490729-734$

Whittet DCB, Schutte WA, Tielens AGGM., Boogert ACA, de Graauw T, Ehrenfreund P, Gerakines PA, Helmich FP, Prusti T and van Dishoeck EF.1996 Astron. Astrophys. 315, L357 - L360

Willacy K, Millar TJ 1998 MNRAS 298/2 562-568

Yang J and Epstein S 1983 Geochim Cosmochim Acta 47 2199-2216

Yang J and Epstein S 1984 Nature 331 544-547

Zahnle KJ and Sleep NH 1997 Comets and the Origin and Evolution of Life 175-208

Zinner E, McKeegan KD and Walker RM 1983 Nature 305 119-121 


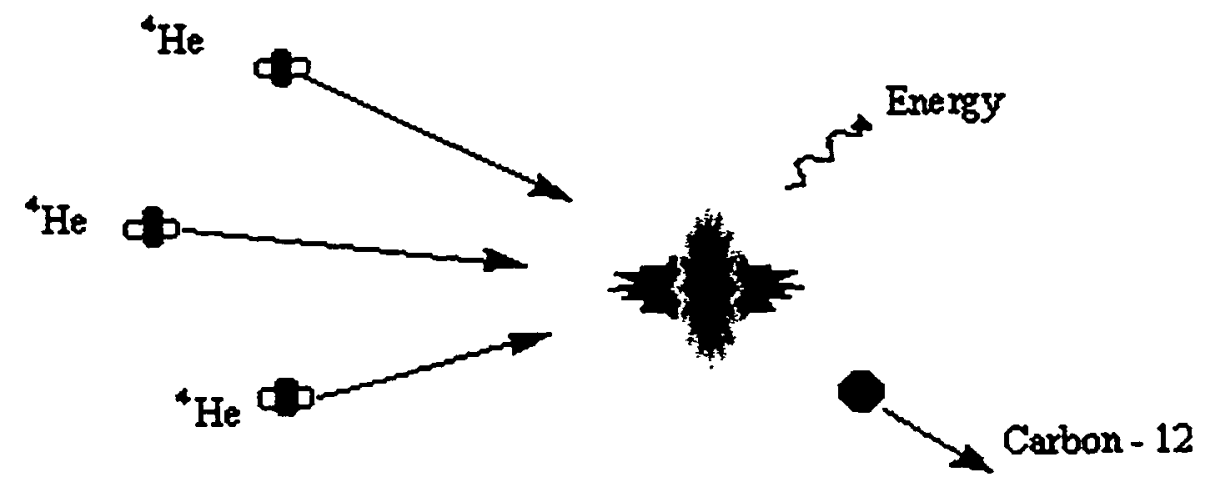

Figure 1 
N

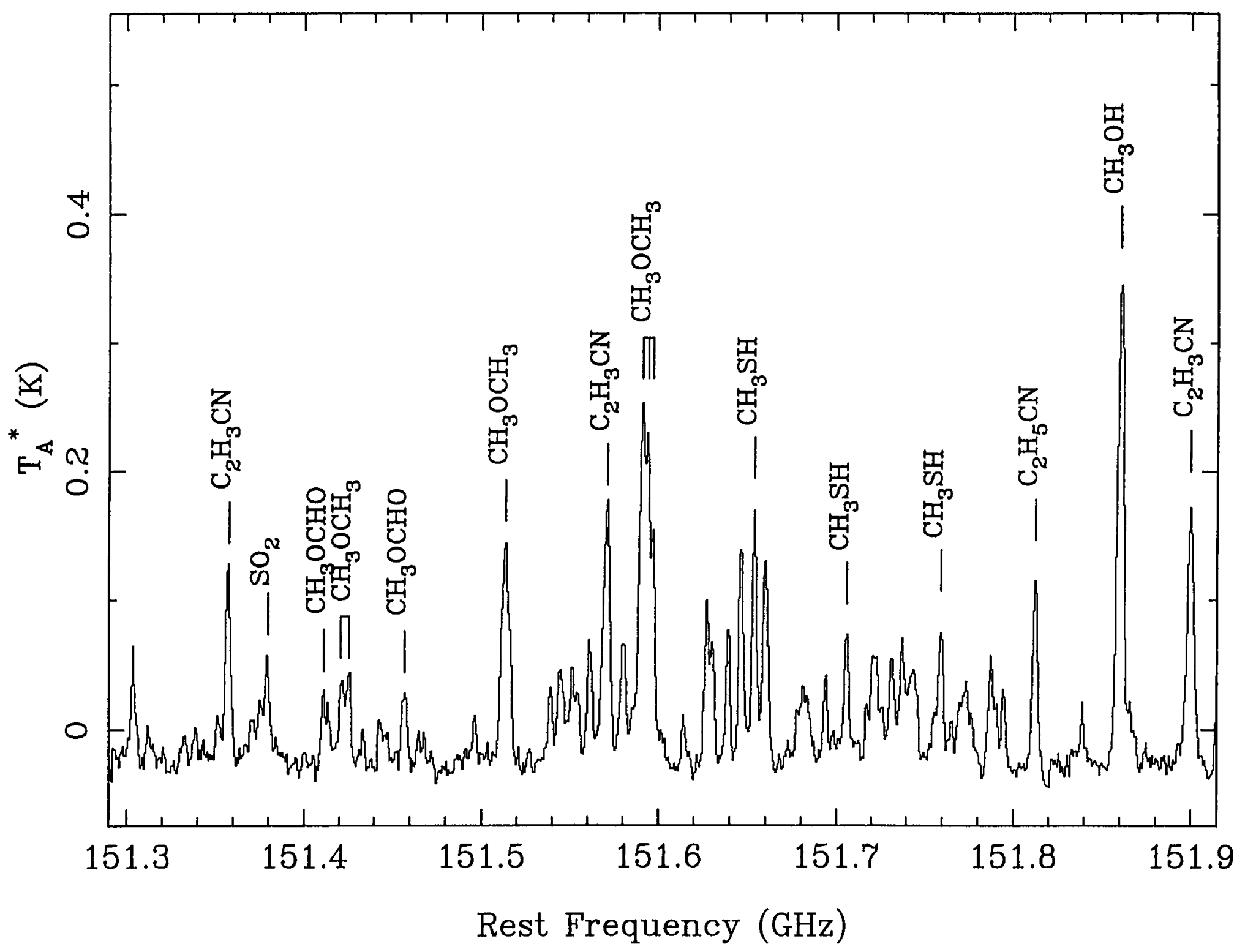




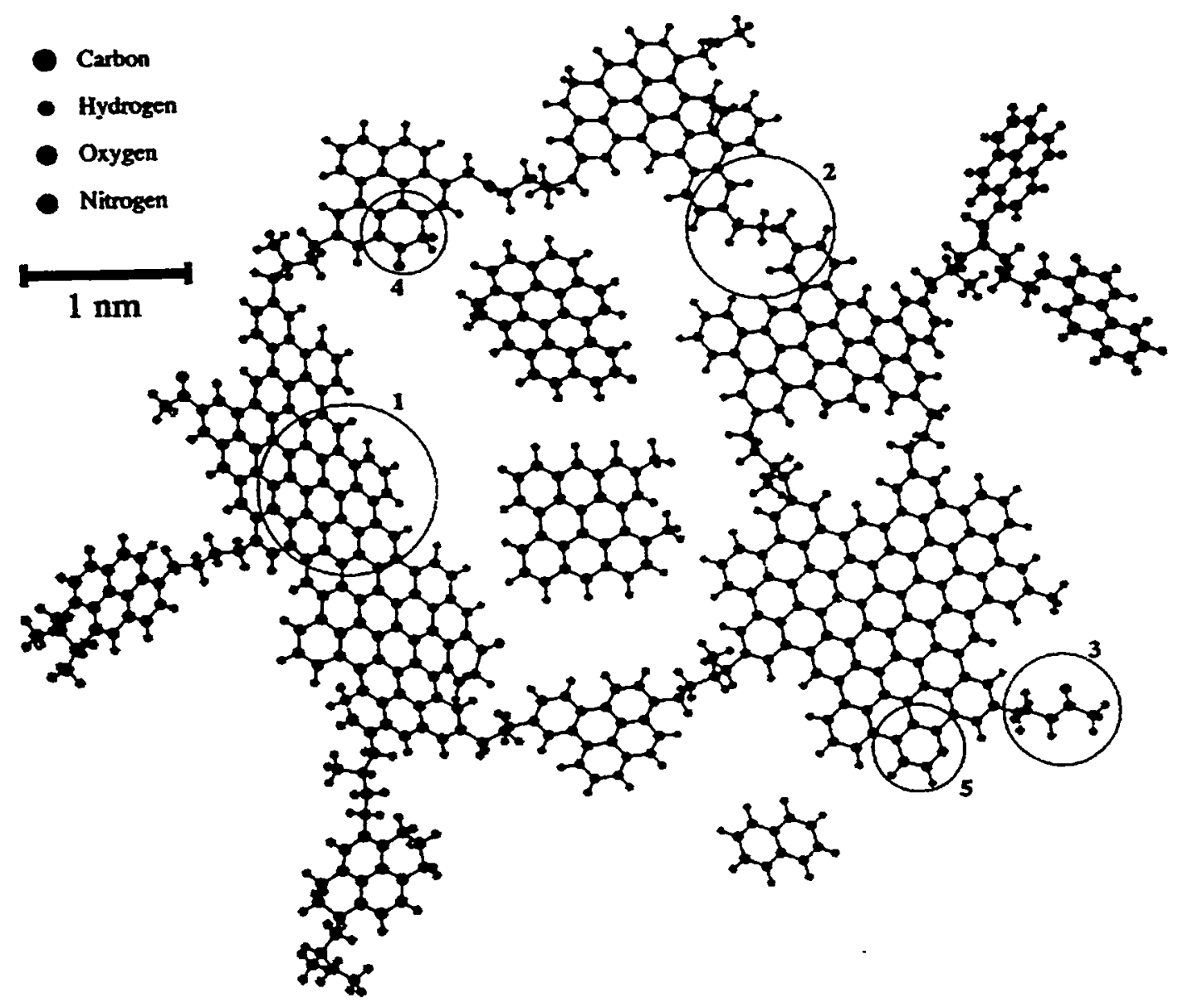

Figure 3 


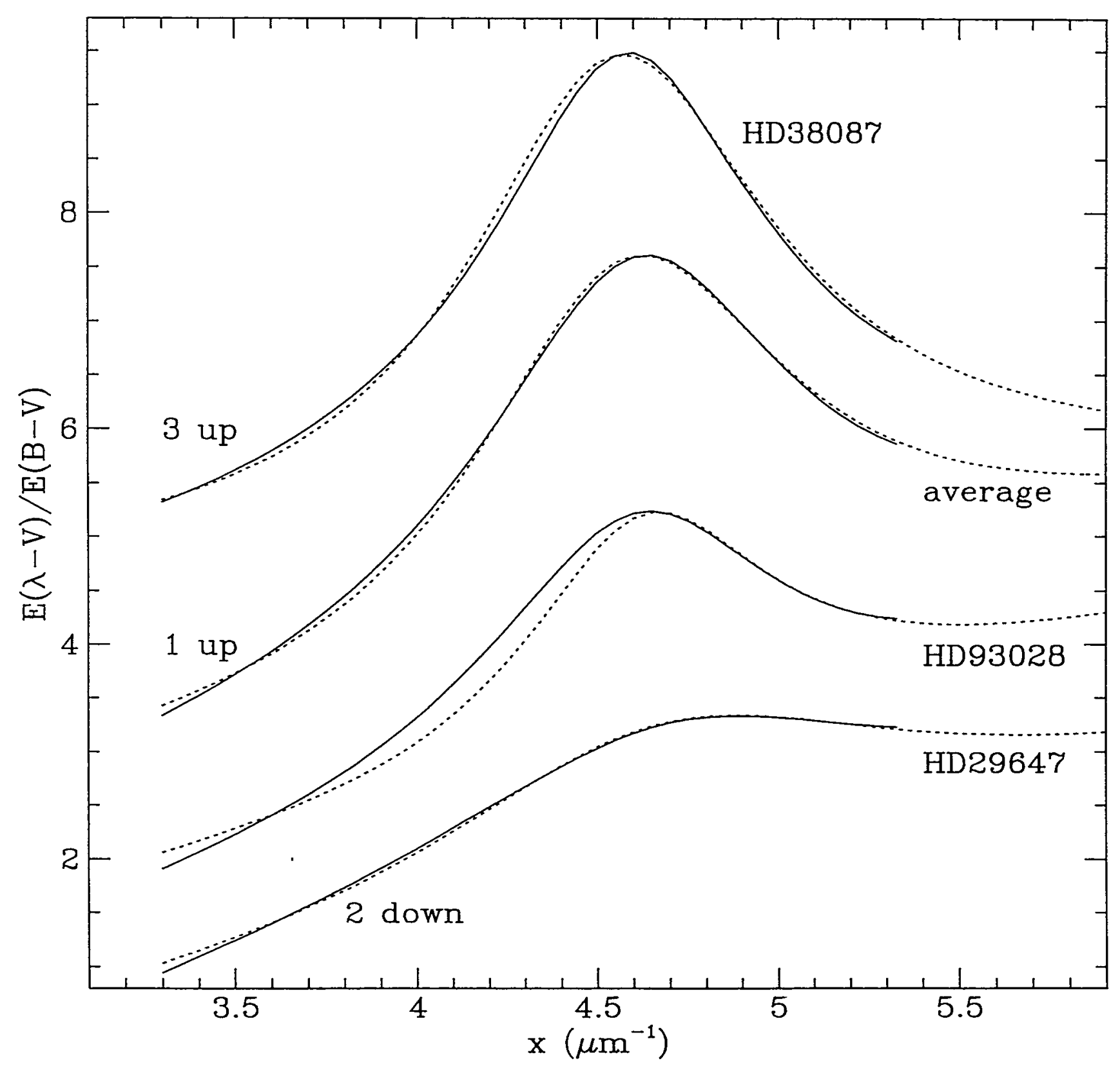

Figure 4 


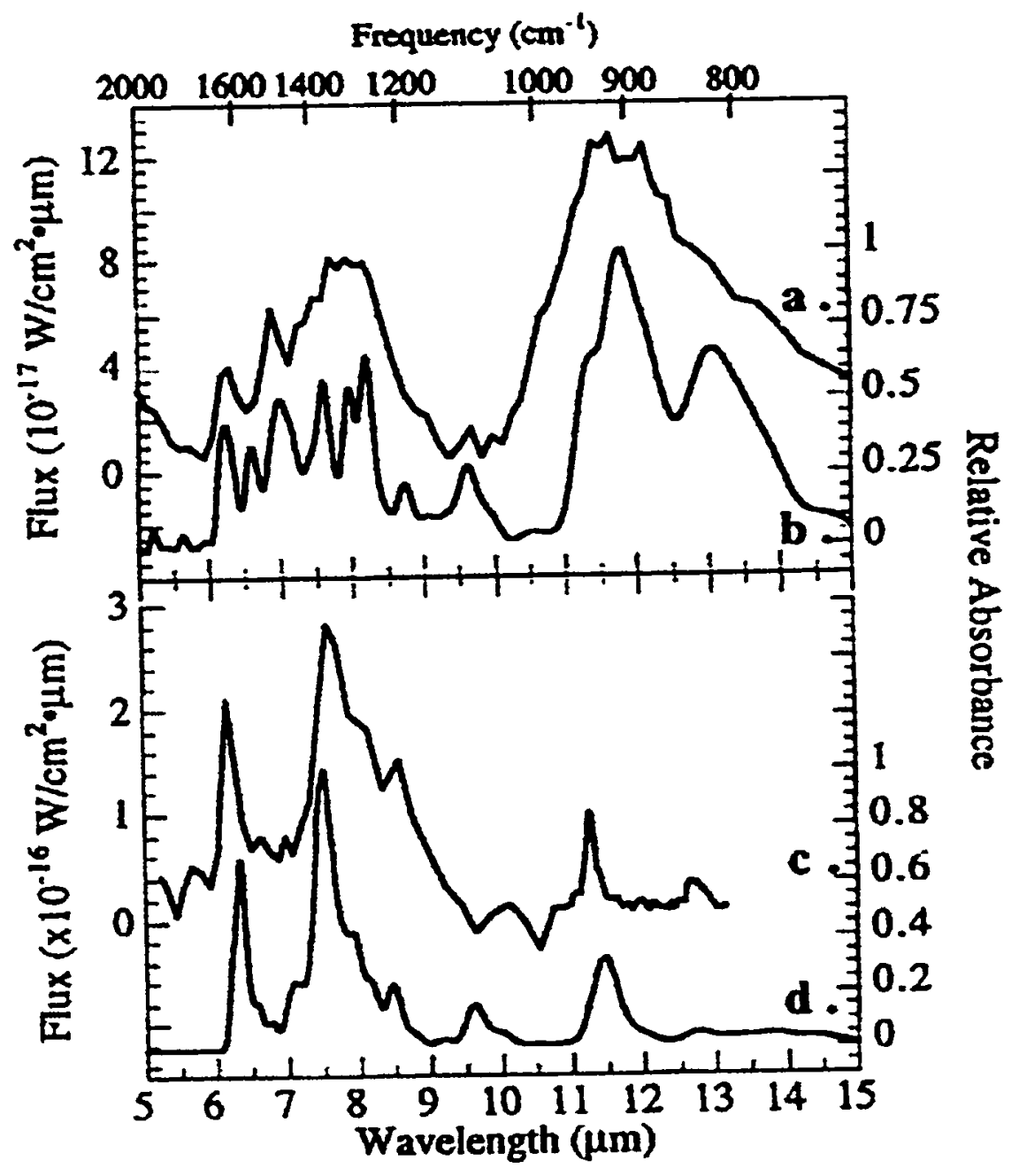

Figure 5 

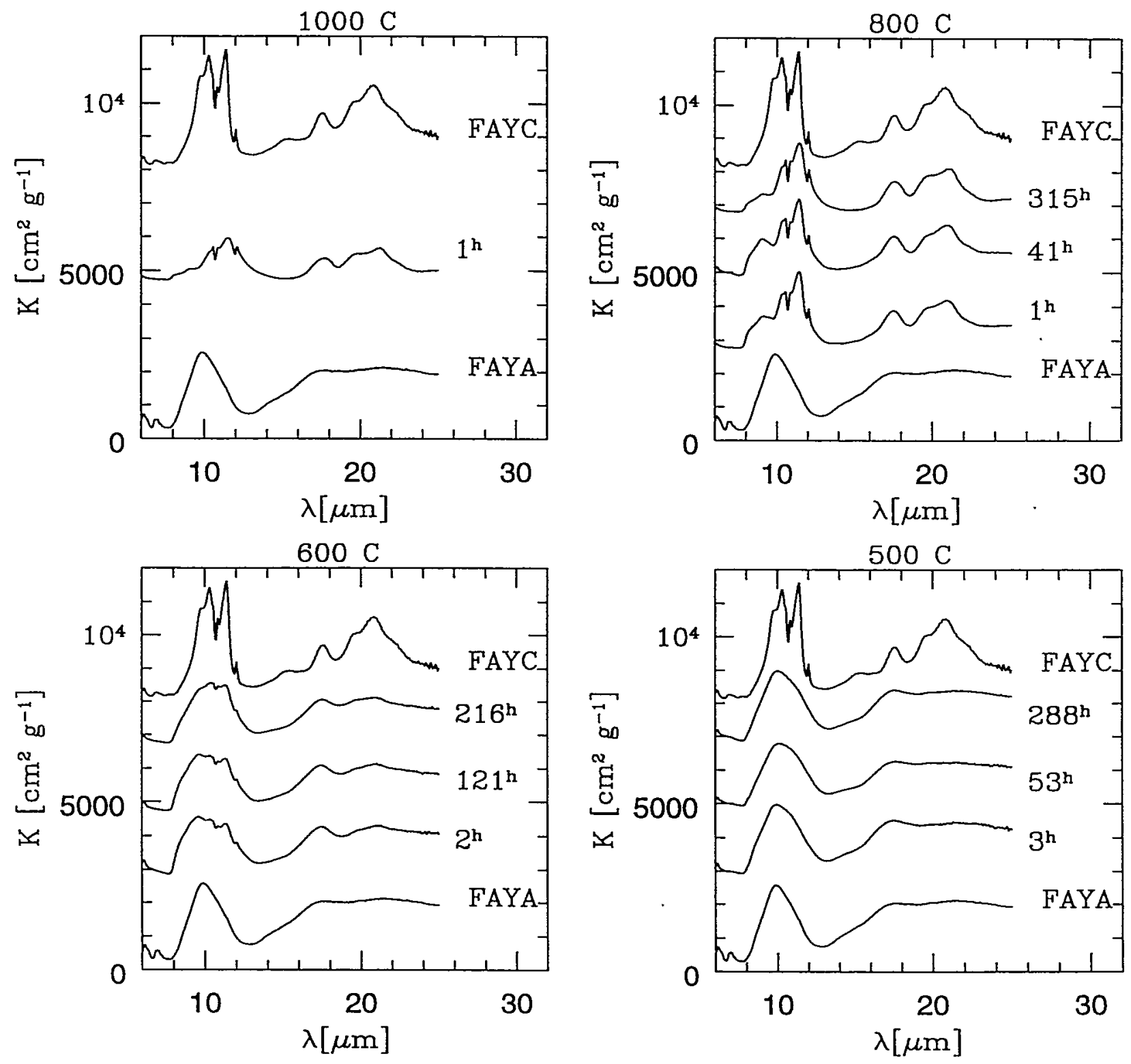

Figure 6 

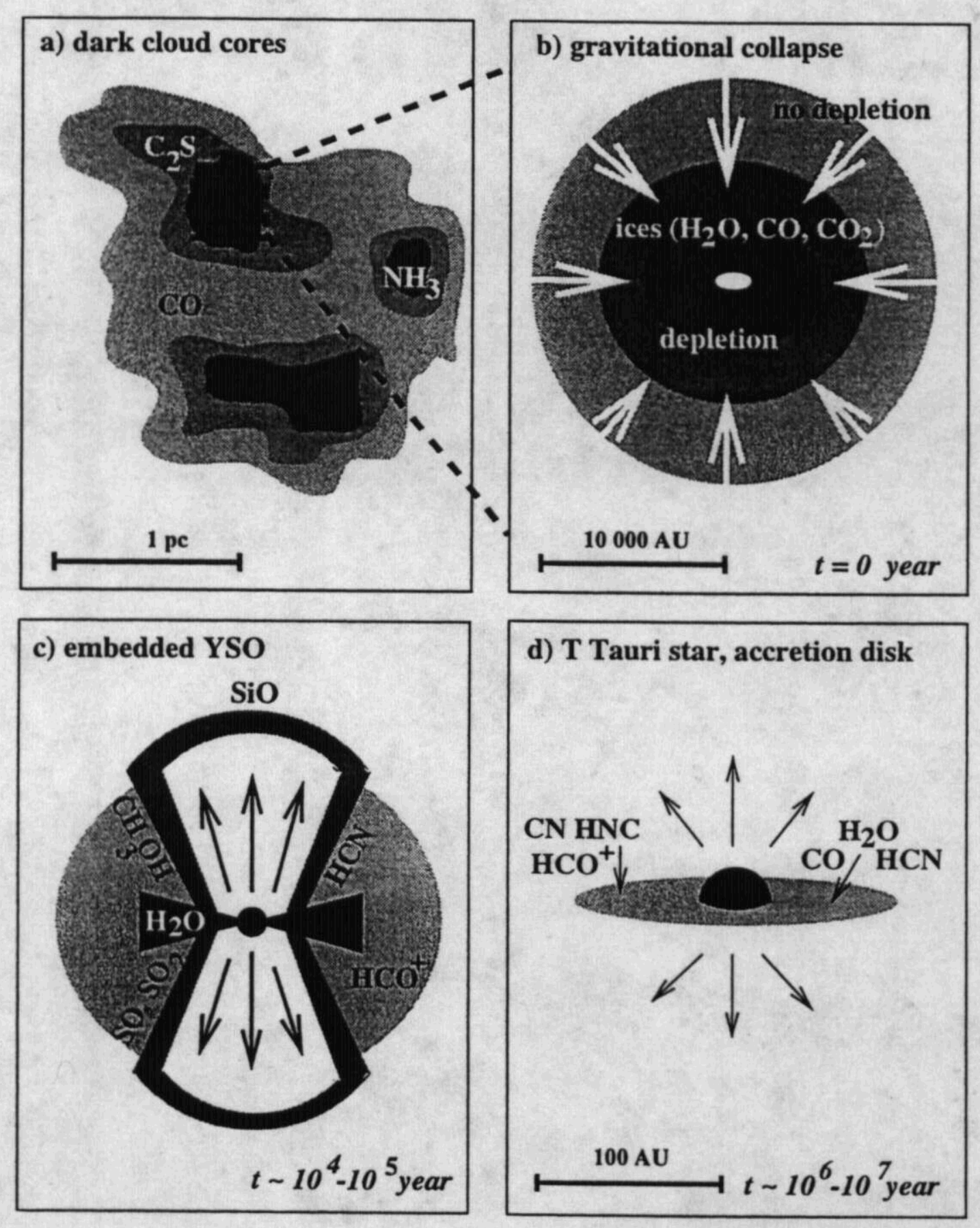

e) main sequence star; planetary system (?)

$\mathrm{H}_{2} \mathrm{O}, \mathrm{CH}_{3} \mathrm{OH}$,

$\mathrm{H}_{2} \mathrm{CO}, \mathrm{HCN}, \ldots$

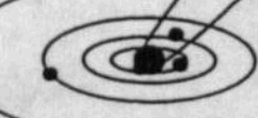

$50 \mathrm{AU}$

$t>10^{7}$ year

\section{Figure 7}




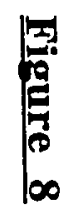

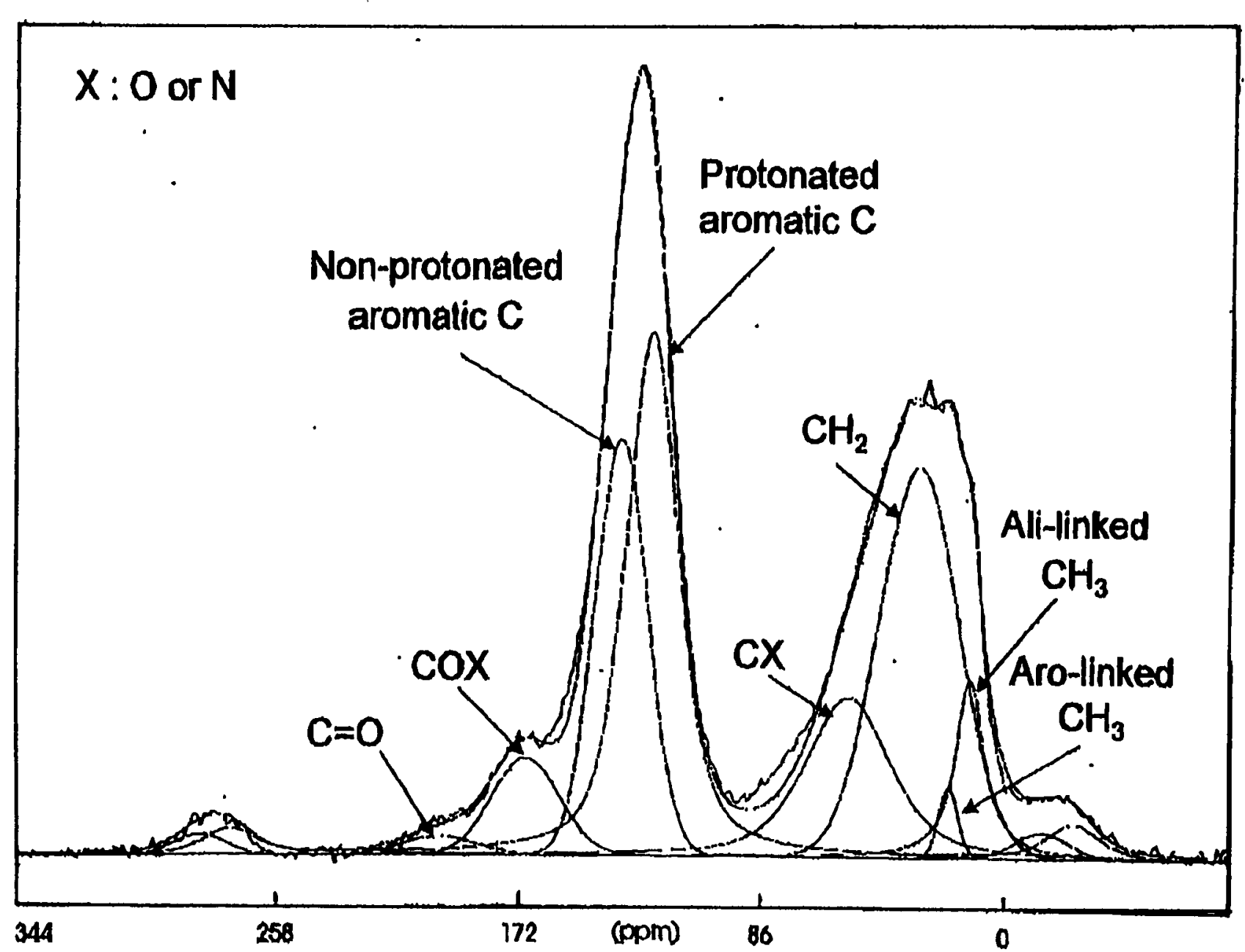




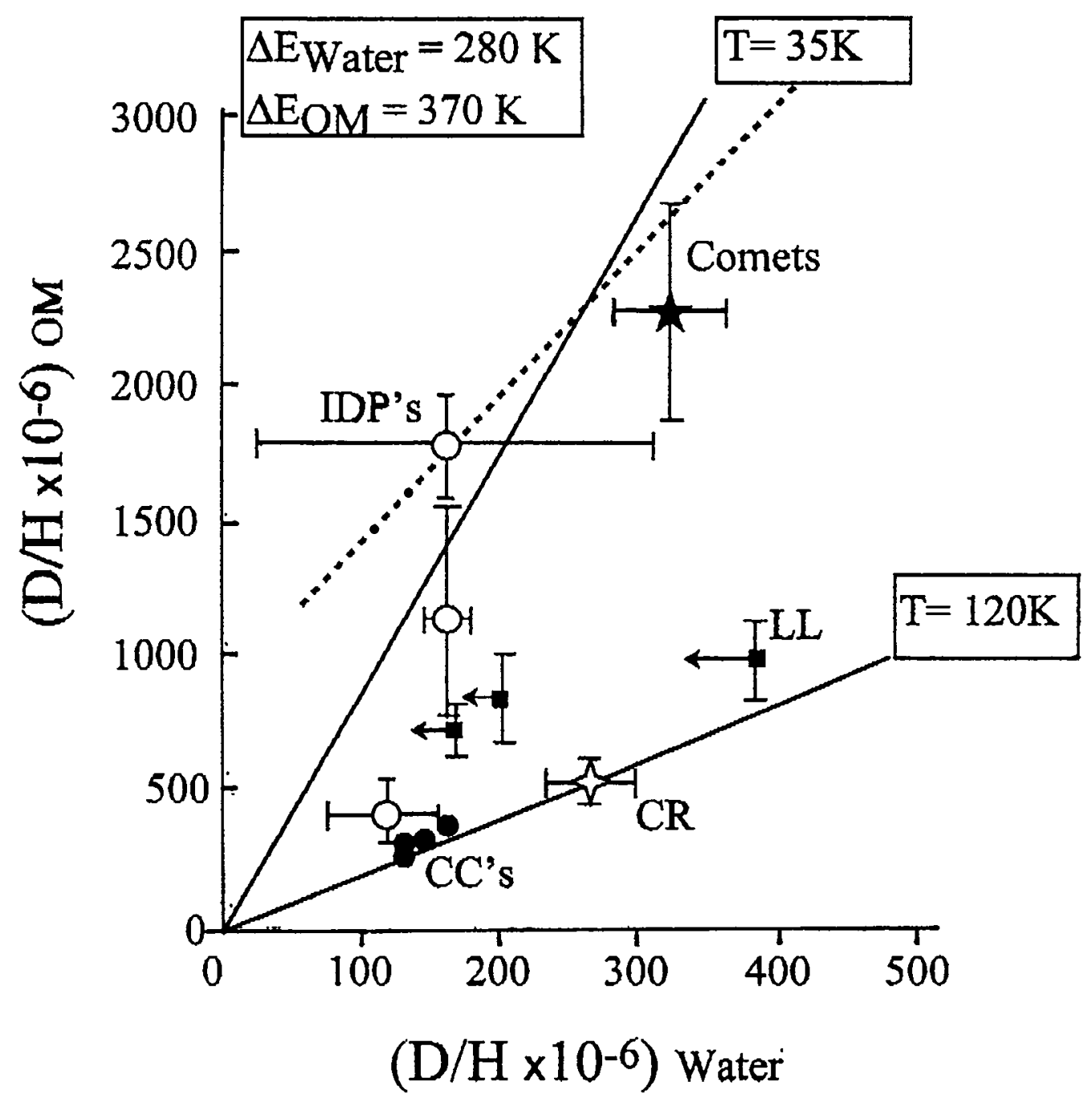

Figure 9 


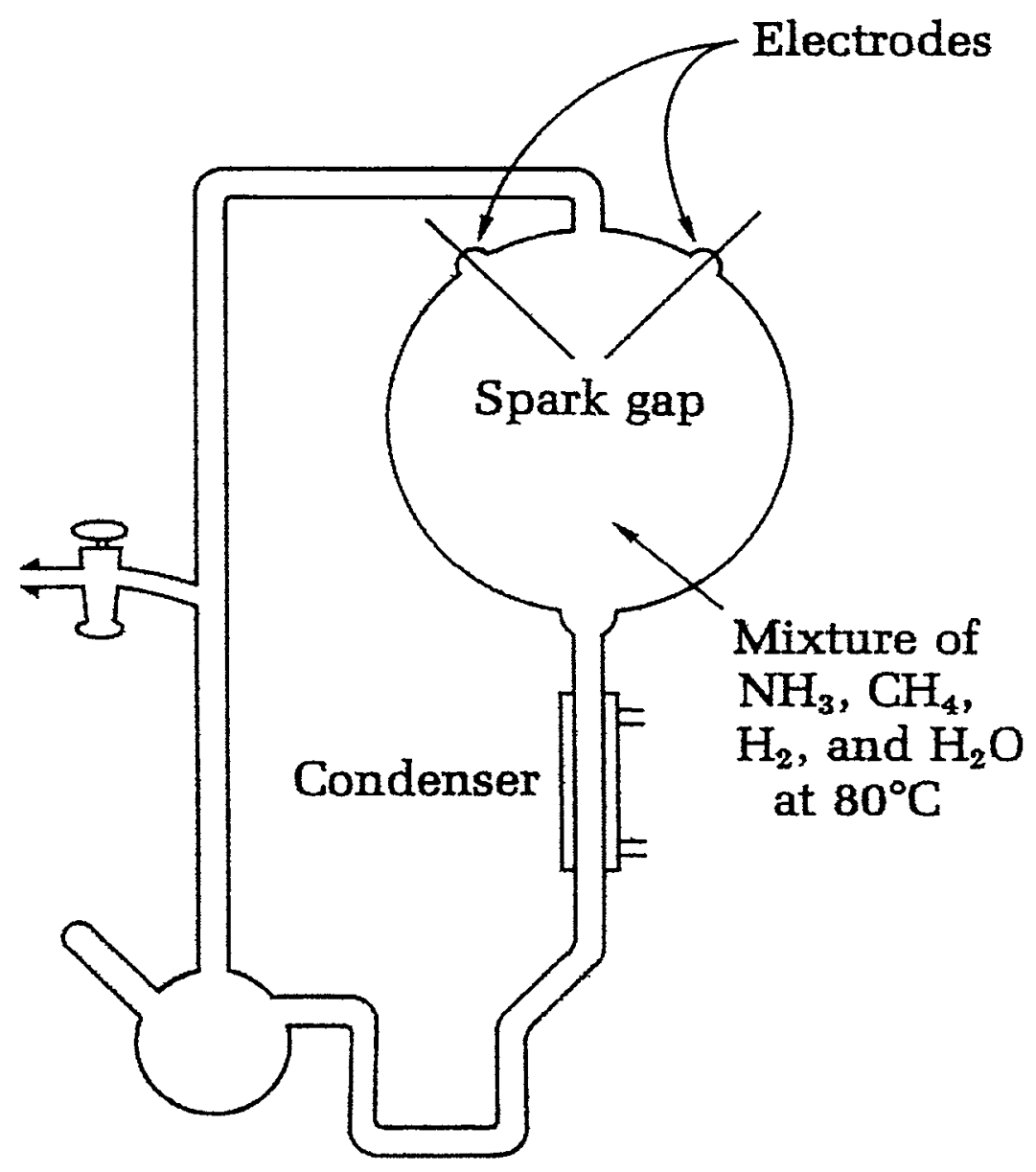

Figure 10 


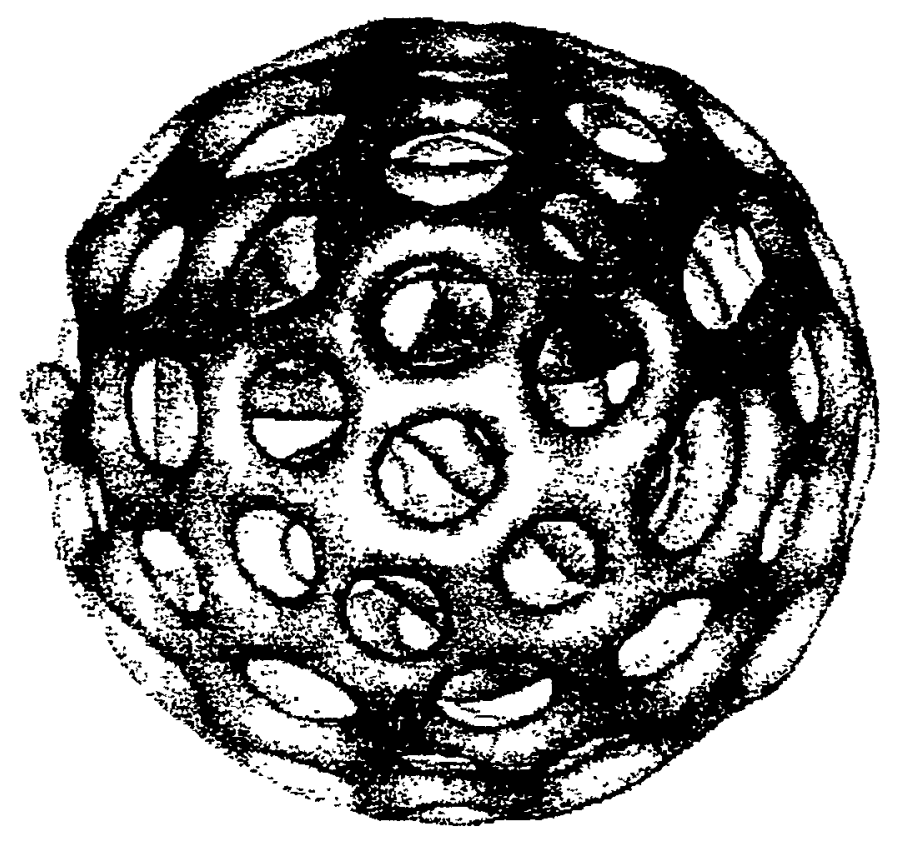

Figure 11 


\begin{tabular}{|c|c|c|c|c|c|c|c|c|c|c|}
\hline \multicolumn{11}{|c|}{ Number of Atoms } \\
\hline 2 & 3 & 4 & 5 & 6 & 7 & 8 & 9 & 10 & 11 & $12+$ \\
\hline $\mathrm{H}_{2}$ & $\mathrm{C}_{3}$ & $c-\mathrm{C}_{3} \mathrm{H}$ & $\mathrm{C}_{5}$ & $\mathrm{C}_{5} \mathrm{H}$ & $\mathrm{C}_{6} \mathrm{H}$ & $\mathrm{CH}_{3} \mathrm{C}_{3} \mathrm{~N}$ & $\mathrm{CH}_{3} \mathrm{C}_{4} \mathrm{H}$ & $\mathrm{CH}_{3} \mathrm{C}_{5} \mathrm{~N}$ ? & $\mathrm{HC}_{9} \mathrm{~N}$ & $\mathrm{C}_{6} \mathrm{H}_{6}$ \\
\hline AIF & $\mathrm{C}_{2} \mathrm{H}$ & 1- $\mathrm{C}_{3} \mathrm{H}$ & $\mathrm{C}_{4} \mathrm{H}$ & $1-\mathrm{H}_{2} \mathrm{C}_{4}$ & $\mathrm{CH}_{2} \mathrm{CHCN}$ & $\mathrm{HCOOCH}_{3}$ & $\mathrm{CH}_{3} \mathrm{CH}_{2} \mathrm{CN}$ & $\left(\mathrm{CH}_{3}\right)_{2} \mathrm{CO}$ & & $\mathrm{HC}_{11} \mathrm{~N}$ \\
\hline $\mathrm{AlCl}$ & $\mathrm{C}_{2} \mathrm{O}$ & $\mathrm{C}_{3} \mathrm{~N}$ & $\mathrm{C}_{4} \mathrm{Si}$ & $\mathrm{C}_{2} \mathrm{H}_{4}$ & $\mathrm{CH}_{3} \mathrm{C}_{2} \mathrm{H}$ & $\mathrm{CH}_{3} \mathrm{COOH}$ ? & $\left(\mathrm{CH}_{3}\right)_{2} \mathrm{O}$ & $\mathrm{NH}_{2} \mathrm{CH}_{2} \mathrm{COOH}$ ? & & PAHs \\
\hline $\mathrm{C}_{2}$ & $\mathrm{C}_{2} \mathrm{~S}$ & $\mathrm{C}_{3} \mathrm{O}$ & 1- $\mathrm{C}_{3} \mathrm{H}_{2}$ & $\mathrm{CH}_{3} \mathrm{CN}$ & $\mathrm{HC}_{5} \mathrm{~N}$ & $\mathrm{C}_{7} \mathrm{H}$ & $\mathrm{CH}_{3} \mathrm{CH}_{2} \mathrm{OH}$ & & & $\mathrm{C}_{60}+?$ \\
\hline $\mathrm{CH}$ & $\mathrm{CH}_{2}$ & $\mathrm{C}_{3} \mathrm{~S}$ & $\mathrm{c}-\mathrm{C}_{3} \mathrm{H}_{2}$ & $\mathrm{CH}_{3} \mathrm{NC}$ & $\mathrm{HCOCH}_{3}$ & $\mathrm{H}_{2} \mathrm{C}_{6}$ & $\mathrm{HC}_{7} \mathrm{~N}$ & & & \\
\hline $\mathrm{CH}^{+}$ & $\mathrm{HCN}$ & $\mathrm{C}_{2} \mathrm{H}_{2}$ & $\mathrm{CH}_{2} \mathrm{CN}$ & $\mathrm{CH}_{3} \mathrm{OH}$ & $\mathrm{NH}_{2} \mathrm{CH}_{3}$ & $\mathrm{HOCH}_{2} \mathrm{CHO}$ & $? \mathrm{C}_{8} \mathrm{H}$ & & & \\
\hline $\mathrm{CN}$ & $\mathrm{HCO}$ & $\mathrm{CH}_{2} \mathrm{D}^{+}$? & $\mathrm{CH}_{4}$ & $\mathrm{CH}_{3} \mathrm{SH}$ & $c-\mathrm{C}_{2} \mathrm{H}_{4} \mathrm{O}$ & & & & & \\
\hline $\mathrm{CO}$ & $\mathrm{HCO}^{+}$ & $\mathrm{HCCN}$ & $\mathrm{HC}_{3} \mathrm{~N}$ & $\mathrm{HC}_{3} \mathrm{NH}^{+}$ & & & & & & \\
\hline $\mathrm{CO}^{+}$ & $\mathrm{HCS}^{+}$ & $\mathrm{HCNH}^{+}$ & $\mathrm{HC}_{2} \mathrm{NC}$ & $\mathrm{HC}_{2} \mathrm{CHO}$ & & & & & & \\
\hline $\mathrm{CP}$ & $\mathrm{HOC}^{+}$ & HNCO & $\mathrm{HCOOH}$ & $\mathrm{NH}_{2} \mathrm{CHO}$ & & & & & & \\
\hline $\mathrm{CSi}$ & $\mathrm{H}_{2} \mathrm{O}$ & HNCS & $\mathrm{H}_{2} \mathrm{CHN}$ & $\mathrm{C}_{5} \mathrm{~N}$ & & & & & & \\
\hline $\mathrm{HCl}$ & $\mathrm{H}_{2} \mathrm{~S}$ & $\mathrm{HOCO}^{+}$ & $\mathrm{H}_{2} \mathrm{C}_{2} \mathrm{O}$ & & & & & & & \\
\hline $\mathrm{KCl}$ & $\mathrm{HNC}$ & $\mathrm{H}_{2} \mathrm{CO}$ & $\mathrm{H}_{2} \mathrm{NCN}$ & & & & & & & \\
\hline $\mathrm{NH}$ & HNO & $\mathrm{H}_{2} \mathrm{CN}$ & $\mathrm{HNC}_{3}$ & & & & & & & \\
\hline NO & $\mathrm{MgCN}$ & $\mathrm{H}_{2} \mathrm{CS}$ & $\mathrm{SiH}_{4}$ & & & & & & & \\
\hline NS & MgNC & $\mathrm{H}_{3} \mathrm{O}^{+}$ & $\mathrm{H}_{2} \mathrm{COH}^{+}$ & & & & & & & \\
\hline $\mathrm{NaCl}$ & $\mathrm{N}_{2} \mathrm{H}^{+}$ & $\mathrm{NH}_{3}$ & & & & & & & & \\
\hline $\mathrm{OH}$ & $\mathrm{N}_{2} \mathrm{O}$ & $\mathrm{SiC}_{3}$ & & & & & & & & \\
\hline PN & $\mathrm{NaCN}$ & $\mathrm{CH}_{3}$ & & & & & & & & \\
\hline so & OCS & & & & & & & & & \\
\hline $\mathrm{SO}^{+}$ & $\mathrm{SO}_{2}$ & & & & & & & & & ' \\
\hline $\operatorname{SiN}$ & $c-\mathrm{SiC}_{2}$ & & & & & & & & & \\
\hline $\mathrm{SiO}$ & $\mathrm{CO}_{2}$ & & & & & & & & & \\
\hline $\mathrm{SiS}$ & $\mathrm{NH}_{2}$ & & & & & & & & & \\
\hline CS & $\mathrm{H}_{3}{ }^{+}$ & & & & & & & & & \\
\hline $\mathrm{HF}$ & $\mathrm{H}_{2} \mathrm{D}^{+}$ & & & & & & & & & \\
\hline
\end{tabular}

Table 1 


\begin{tabular}{|c|c|c|c|}
\hline Process & Space & Laboratory (max) & Units \\
\hline \multirow[t]{2}{*}{ UV irradiation } & $3 \times 10^{25}$ - diffuse ISM $\left(10^{9} \mathrm{yr}.\right)$ & \multirow[t]{2}{*}{$4 \times 10^{22}$} & \multirow[t]{2}{*}{$\mathrm{EV} \mathrm{cm^{-2 }}$} \\
\hline & $4 \times 10^{20}$ - dense ISM $\left(10^{9} \mathrm{yr}\right.$. $)$ & & \\
\hline \multirow[t]{4}{*}{ Ion bombardment } & $3 \times 10^{3}$ - diffuse ISM $\left(10^{9} \mathrm{yr}\right)$ & \multirow[t]{4}{*}{660} & \multirow[t]{4}{*}{ eV mol-1 } \\
\hline & $3 \times 10^{2}-$ dense ISM $\left(10^{9} \mathrm{yr}\right)$ & & \\
\hline & $1 \times 10^{6}$ - pre-comet & & \\
\hline & $6 \times 10^{2}-$ comet & & \\
\hline $\mathrm{H}$ atoms & $8 \times 10^{21}$ & $6.9 \times 10^{19}$ & $\mathrm{~cm}^{-2}$ \\
\hline \multirow[t]{2}{*}{ Thermal } & 1000 - stellar outflows & \multirow[t]{2}{*}{1300} & \multirow[t]{2}{*}{$\mathrm{K}$} \\
\hline & 100 - pre-solar nebula & & \\
\hline
\end{tabular}

Table 2 


\begin{tabular}{|c|c|c|c|c|}
\hline Species & $\mathrm{X}$ & Trans. & $S_{\nu}$ & $f$ \\
\hline${ }^{12} \mathrm{CO}$ & $8(-5)$ & $2-1$ & $13.95(.43)$ & \\
\hline${ }^{13} \mathrm{CO}$ & $4(-6)$ & $2-1$ & $5.40(.13)$ & 3 \\
\hline $\mathrm{C}^{18}$ & $4(-7)$ & $2-1$ & $.68(.07)$ & 5 \\
\hline \multirow[t]{2}{*}{$\mathrm{HCN}$} & \multirow[t]{2}{*}{$2(-8)$} & $1-0$ & $.38(.05)$ & 40 \\
\hline & & $3-2$ & $<1.9$ & \\
\hline $\mathrm{HNC}$ & $2(-8)$ & $1-0$ & $.16(.03)$ & 90 \\
\hline \multirow[t]{2}{*}{$\mathrm{CN}$} & \multirow[t]{2}{*}{$3(-8)$} & $1-0$ & $1.38(.24)$ & 10 \\
\hline & & $2-1$ & $8.7(0.4)$ & \\
\hline \multirow[t]{2}{*}{ CS } & \multirow[t]{2}{*}{$1(-8)$} & $3-2$ & $.47(.07)$ & 30 \\
\hline & & $5-4$ & $.67(.11)$ & \\
\hline \multirow[t]{3}{*}{$\mathrm{H}_{2} \mathrm{CO}$} & \multirow[t]{3}{*}{$2(-8)$} & $2_{12}-1_{11-0}$ & $.30(.04)$ & 50 \\
\hline & & $2_{02}-1_{01-p}$ & $.11(.04)$ & 100 \\
\hline & & $3_{13}-2_{12}-0$ & $.48(.04)$ & 100 \\
\hline $\mathrm{C}_{2} \mathrm{H}$ & $8(-8)$ & $1-0$ & $.55(.08)$ & 7 \\
\hline \multirow[t]{2}{*}{$\mathrm{HCO}^{+}$} & \multirow[t]{2}{*}{$8(-9)$} & $1-0$ & $.82(.04)$ & 11 \\
\hline & & $3-2$ & $4.1(.5)$ & \\
\hline \multicolumn{5}{|l|}{ Undetected } \\
\hline $\mathrm{C}^{34}$ & $5(-10)$ & $5-4$ & $.38(.11)$ & $\geq 6$ \\
\hline $\mathrm{H}^{13} \mathrm{CO}^{+}$ & $1.1(-10)$ & $1-0$ & $<.06$ & $\geq 3$ \\
\hline $\mathrm{N}_{2} \mathrm{H}^{+}$ & $5(-10)$ & $1-0$ & $<.45$ & $\geq 2$ \\
\hline \multirow[t]{2}{*}{$\mathrm{SiO}$} & \multirow[t]{2}{*}{$\leq 4(-12)$} & $2-1$ & $<.13$ & \\
\hline & & $5-4$ & $<.36$ & \\
\hline \multirow[t]{2}{*}{ SiS } & \multirow[t]{2}{*}{$1(-11)$} & $5-4$ & $<.09$ & \\
\hline & & $8-7$ & $<.09$ & \\
\hline $\mathrm{H}_{2} \mathrm{~S}$ & $\leq 5(-10)$ & $1_{10}-1_{01}$ & $<.50$ & \\
\hline $\mathrm{C}_{3} \mathrm{H}_{2}$ & $\leq 4(-10)$ & $1_{10}-1_{01}$ & $<.14$ & \\
\hline \multirow{2}{*}{$\mathrm{HC}_{3} \mathrm{~N}$} & \multirow[t]{2}{*}{$6(-9)$} & $10-9$ & $<.07$ & \\
\hline & & $16-15$ & $<.15$ & \\
\hline $\mathrm{CH}_{3} \mathrm{OH}$ & $2(-9)$ & $3_{0}-2_{0}$ & $<.24$ & \\
\hline $\mathrm{CO}^{+}$ & $\leq 1(-11)$ & $2-1$ & $<.54$ & \\
\hline $\mathrm{SO}$ & & $66_{5}-5_{4}$ & $<.28$ & \\
\hline $\mathrm{SO}_{2}$ & & $25_{323^{-}}-24_{420}$ & $<.16$ & \\
\hline $\mathrm{SiC}_{2}$ & & $4_{04}-3_{03}$ & $<.15$ & \\
\hline HNCS & & $12_{012^{-}} 11_{011}$ & $<.23$ & \\
\hline $\mathrm{HCO}$ & & $1_{10}-0_{00}$ & $<.12$ & \\
\hline $\mathrm{HCOOCH}_{3}$ & & $20_{020}-19_{019}$ & $<.40$ & \\
\hline $\mathrm{HOHCS}^{+}$ & & $2-1$ & $<.14$ & \\
\hline
\end{tabular}

Table 3 
Molecule

$\mathrm{H}_{2} \mathrm{O}$
$\mathrm{H}_{2} \mathrm{O}_{2}$
$\mathrm{CO}$
$\mathrm{CO}_{2}$

$\mathrm{CH}_{4}$
$\mathrm{C}_{2} \mathrm{H}_{2}$
$\mathrm{C}_{2} \mathrm{H}_{6}$
$\mathrm{CH}_{3} \mathrm{C}_{2} \mathrm{H}$

$\mathrm{CH}_{3} \mathrm{OH}$

$\mathrm{H}_{2} \mathrm{CO}$

$\mathrm{HCOOH}$

$\mathrm{HCOOCH}_{3}$

$\mathrm{CH}_{3} \mathrm{CHO}$

$\mathrm{H}_{2} \mathrm{CCO}$

$\mathrm{C}_{2} \mathrm{H}_{5} \mathrm{OH}$

$\mathrm{CH}_{3} \mathrm{OCH}_{3}$

$\mathbf{N H}_{3}$

HCN

HNCO

HINC

$\mathrm{CH}_{3} \mathrm{CN}$

$\mathrm{HC}_{3} \mathbf{N}$

$\mathrm{NH}_{2} \mathrm{CHO}$

Glycine

$\mathrm{CH}_{2} \mathrm{NH}$

HCFN

$\mathrm{H}_{2} \mathrm{~S}$

SO

$\mathrm{CS}_{2}$

$\mathrm{SO}_{2}$

$\mathrm{H}_{2} \mathrm{CS}$

$\mathbf{S}_{\mathbf{2}}$

$\mathrm{NaCl}$

$\mathrm{NaOH}$
Hyakutake Hale-Bopp Notes

100

$$
<0.04
$$

6-30

$<7$

20

6

a)

c)

$\begin{array}{ll}0.7 & 0.6 \\ \sim 0.5 & 0.1 \\ 0.4 & 0.3 \\ - & <0.045 \\ & \\ 0.2-1 & 2.4 \\ - & 1.1 \\ - & 0.08 \\ - & 0.08 \\ - & 0.02 \\ - & <0.032 \\ - & <0.05 \\ & <0.45\end{array}$

0.5

0.1

0.07

0.7

0.25

0.06

0.04

0.02

0.02

0.01

$<0.5$

$<0.032$

$<0.003$

0.8

1.5

0.1

0.3

0.1

$0.2-0.8$

0.2

0.1

$-$

$\overline{0.005}$

0.02

$-\quad<0.0008$

$<0.0003$ a)

a)

a)

a)

b)

\section{Table 4}




\begin{tabular}{|c|c|c|}
\hline \multicolumn{2}{|c|}{ Compound Class } & Concentration (ppm) \\
\hline \multicolumn{3}{|c|}{ Amino Acids } \\
\hline & $\mathrm{CM}$ meteorites & $17-60$ \\
\hline & CI meteorites & $\sim 5^{a)}$ \\
\hline \multicolumn{2}{|c|}{ Aliphatic hydrocarbons } & $>35$ \\
\hline \multicolumn{2}{|c|}{ Aromatic hydrocarbons } & $3319^{b)}$ \\
\hline \multicolumn{2}{|c|}{ Fullerenes } & $>100^{c)}$ \\
\hline \multicolumn{2}{|c|}{ Carboxylic acids } & $>300$ \\
\hline \multicolumn{2}{|c|}{ Hydroxycarboxylic acids } & 15 \\
\hline \multicolumn{2}{|c|}{ Dicarboxylic acids \& Hydroxydicarboxylic acids } & 14 \\
\hline \multicolumn{2}{|c|}{ Purines \& Pyrimidines } & 1.3 \\
\hline \multicolumn{2}{|c|}{ Basic N-heterocycles } & 7 \\
\hline \multicolumn{2}{|c|}{ Amines } & 8 \\
\hline Amides & $\begin{array}{l}\text { linear } \\
\text { cyclic }\end{array}$ & $\begin{array}{r}>70 \\
>2^{d)}\end{array}$ \\
\hline \multicolumn{2}{|c|}{ Alcohols } & 11 \\
\hline \multicolumn{2}{|c|}{ Aldehydes \& Ketones } & 27 \\
\hline \multicolumn{2}{|c|}{ Sulphonic acids } & 68 \\
\hline \multicolumn{2}{|c|}{ Phosphonic acids } & 2 \\
\hline
\end{tabular}

\section{Table 5}


Major Sources (in kg/yr) of Prebiotic Organic Compounds in the Early Earth

\begin{tabular}{|ll|}
\hline Terrestrial Sources & $\mathrm{kg} / \mathrm{yr}^{*}$ \\
\hline UV Photolysis $^{1}$ & $3 \times 10^{8}$ \\
Electric Discharge $^{2}$ & $3 \times 10^{7}$ \\
Shocks from impacts $^{3}$ & $4 \times 10^{2}$ \\
Hydrothermal Vents $^{4}$ & $1 \times 10^{8}$ \\
\hline Extraterrestrial Sources & \\
\hline IDPs & \\
Comets & $2 \times 10^{8}$ \\
\hline Total & $1 \times 10^{11}$ \\
\hline
\end{tabular}

Table 6 